\title{
DE-FC26-04NT42240
}

Ultra Deep Wave Equation Imaging and Illumination

Final Report for the Period October 2004 - August 2006

August 2006

Alexander M. Popovici

Sergey Fomel

Paul Sava

Sean Crawley

Yining Li

Cristian Lupascu

3DGeo Development Inc.

17171 Park Row, Suite 247

Houston, TX 77084 


\section{Project Title: Ultra Deep Wave Equation Imaging and Illumination}

Type of Report: Department of Energy Final Report

Award Dates:

Effective Date: $\quad$ 10/01/2004

End of Grant Support: 08/01/2006

Authors: Alexander M. Popovici, Sergey Fomel, Paul Sava, Sean Crawley, Yining Li, Cristian Lupascu

Date of Report: November 2006

Award Number: DE-FC26-04NT42240

Program Manager: Frances Toro

PI Name: Alexander Mihai Popovici, Ph.D.

Company:

3DGeo Development Inc.

17171 Park Row, Suite 247

Houston, TX 77084

Tel: 281 579-9712

Fax: 281 579-9716 


\section{Disclaimer}

This report was prepared as an account of work sponsored by an agency of the United States Government. Neither the United States government nor any agency thereof, nor any of their employees, makes any warranty, express or implied, or assumes any legal liability or responsibility for the accuracy, completeness, or usefulness of any information, apparatus, product, or process disclosed, or represents that its use would not infringe privately owned rights. Reference herein to any specific commercial product, process, or service by trade name, trademark, manufacturer, or otherwise does not necessarily constitute or imply its endorsement, recommendation, or favoring by the United States Government or any agency thereof. The views and opinions of authors expressed herein do not necessarily state or reflect those of the United States Government or any agency thereof. 


\section{ABSTRACT}

In this project we developed and tested a novel technology, designed to enhance seismic resolution and imaging of ultra-deep complex geologic structures by using state-of-the-art waveequation depth migration and wave-equation velocity model building technology for deeper data penetration and recovery, steeper dip and ultra-deep structure imaging, accurate velocity estimation for imaging and pore pressure prediction and accurate illumination and amplitude processing for extending the AVO prediction window. Ultra-deep wave-equation imaging provides greater resolution and accuracy under complex geologic structures where energy multipathing occurs, than what can be accomplished today with standard imaging technology.

The objective of the research effort was to examine the feasibility of imaging ultra-deep structures onshore and offshore, by using (1) wave-equation migration, (2) angle-gathers velocity model building, and (3) wave-equation illumination and amplitude compensation. The effort consisted of answering critical technical questions that determine the feasibility of the proposed methodology, testing the theory on synthetic data, and finally applying the technology for imaging ultra-deep real data. Some of the questions answered by this research addressed: (1) the handling of true amplitudes in the downward continuation and imaging algorithm and the preservation of the amplitude with offset or amplitude with angle information required for AVO studies, (2) the effect of several imaging conditions on amplitudes, (3) non-elastic attenuation and approaches for recovering the amplitude and frequency, (4) the effect of aperture and illumination on imaging steep dips and on discriminating the velocities in the ultra-deep structures. All these effects were incorporated in the final imaging step of a real data set acquired specifically to address ultra-deep imaging issues, with large offsets $(12,500 \mathrm{~m})$ and long recording time $(20 \mathrm{~s})$. 


\section{Table of Contents}

\begin{tabular}{|c|c|}
\hline & STRAC \\
\hline & 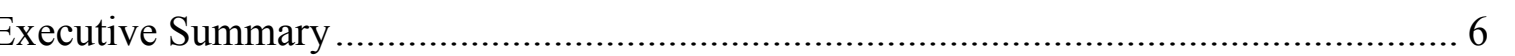 \\
\hline & 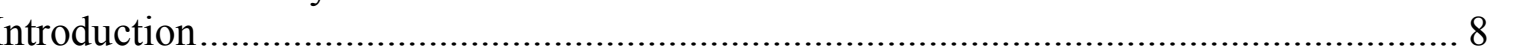 \\
\hline & ltra Deep Wave Equation Imaging and Illumination .... \\
\hline & Task 1: Image true amplitude in wave-equation migration .. \\
\hline & True amplitude imaging theory......................................... \\
\hline & FROM GEOMETRICAL TO WAVE EQUATION EXTRAPOLATION $. . . \ldots \ldots \ldots . . . .14$ \\
\hline & GEOMETRICAL AMPLITUDES AND TRAVELTIME DERIVATIVES ............ 15 \\
\hline & 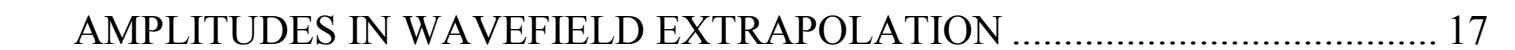 \\
\hline & Building the benchmark model and benchmark dataset ..................................... 19 \\
\hline & AMPLITUDES IN KIRCHHOFF AND WAVE-EQUATION MIGRATION... \\
\hline & Task 2: Implement an alternative wave-equation amplitude imaging condition...... \\
\hline & Conventional imaging condition............. \\
\hline & Space-shift imaging condition ..... \\
\hline & 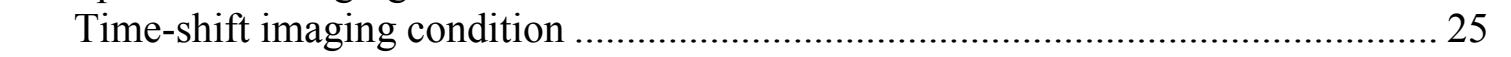 \\
\hline & ANGLE TRANSFORMATION IN WAVE-EQUATION IMAGING ........ \\
\hline & 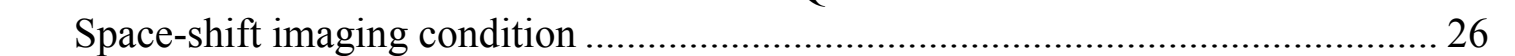 \\
\hline & Time-shift imaging condition ..... \\
\hline & EXAMPLES …............................. \\
\hline & Task 3: Develop amplitude compensation for irregular ill \\
\hline & REFERENCES \\
\hline
\end{tabular}




\section{Executive Summary}

The work performed under the DoE NETL project developed and tested a novel, next generation technology, designed to enhance seismic resolution and imaging of ultra-deep complex geologic structures by using (1) wave-equation depth migration and (2) wave-equation velocity model building technology for deeper data penetration and recovery, steeper dip and ultra-deep structure imaging, and accurate velocity estimation for imaging and pore pressure prediction, and (3) accurate illumination and amplitude processing for extending the AVO prediction window. Ultra-deep wave-equation imaging provides much greater resolution and accuracy than what can be accomplished with standard imaging technology. The advanced imaging methodology may improve the success rate and cost effectiveness for new deep-field discoveries, and also has applications in increasing recovery efficiency for the development of existing fields.

The goal of the project was to develop and test a novel technology designed to enhance seismic resolution and imaging of ultra-deep water complex geologic structures by using wave-equation depth migration, wave-equation velocity model building, and wave-equation illumination. The main objectives of the research effort are centered around several research areas: (1) waveequation migration, (2) angle-gathers velocity model building, and (3) wave-equation illumination and amplitude compensation. These research directions were addressed by several tasks that are listed below. The first group of tasks were centered around improving the theory and understanding of several aspects related to wave-propagation in deeper structures. The theory was then tested on synthetic data and finally applied on real data. The following tasks were executed during this project and will be described in detail in the following sections:

1. Confirm our ability to image true amplitude in wave-equation migration.

2. Implement alternative wave-equation amplitude imaging conditions.

3. Develop amplitude compensation for irregular illumination.

4. Investigate non-elastic attenuation and suggest approaches for recovery.

5. Demonstrate imaging and accuracy on POC-2 ultra-deep data.

6. Investigate effect of aperture on ultra-deep data.

7. Investigate the effect of large offsets on velocity discrimination on ultra-deep data.

The tasks were designed to answer critical technical questions that would determine the feasibility of the proposed methodology. Some of the questions answered by this research addressed: (1) the handling of true amplitudes in the downward continuation and imaging algorithm and the preservation of the amplitude with offset or amplitude with angle information required for AVO studies, (2) the effect of several imaging conditions on amplitudes, (3) nonelastic attenuation and approaches for recovering the amplitude and frequency, (4) the effect of aperture and illumination on imaging steep dips and on discriminating the velocities in the ultradeep structures. All these effects were incorporated in the final imaging step of a real data set acquired specifically to address ultra-deep imaging issues, with large offsets $(12,500 \mathrm{~m})$ and long recording time $(20 \mathrm{~s})$.

True-amplitude imaging is necessary when the amplitudes of the seismic image are used as input for estimating the petrophysical properties of the reservoir rocks. Seismic imaging of deep targets requires a particular attention to amplitude preservation. Seismic signals are attenuated and scattered during propagation to deep targets. Compensating for the signal loss and for an irregular illumination of exploration targets at depth becomes a necessity both for obtaining a 
reliable structural image and for obtaining an image with meaningful amplitudes. In structurally complex media, both the single-arrival assumption and the classical asymptotic theory of Kirchhoff integrals become inadequate. Wave-equation imaging is identified a preferable alternative to the Kirchhoff method because of its ability to handle multiple arrivals, large velocity variations, and limited bandwidth wave-propagation effects. However, the theory of amplitude preservation in wave-equation imaging is less understood, and practical implementations still lack reliable tools of amplitude compensation. There are four parts of the amplitude compensation problem in wave-equation imaging:

1. Preserving true wave amplitudes at the downward wave-propagation step. True amplitudes are not automatically preserved by wave-equation methods, because the oneway way equation, which serves as the basis for most of them, does not preserve the correct two-way equation amplitudes. Amplitude preservation at this step is especially important for imaging steeply dipping reflectors such as salt flanks and faults.

2. Recovering the true reflectivity (as a function of reflection angle) through applying the appropriate imaging condition. The overburden propagation effects should be removed at this step, recovering the true reflectivity at the image point.

3. Compensating for irregular target illumination resulting from incomplete seismic acquisition geometries. This step is crucial for imaging under salt bodies and in other difficult areas of deep exploration. Wave-equation methods (unlike ray-tracing methods) offer the additional benefit that the illumination can be studied for individual frequency bandwidths.

4. Compensating for non-elastic attenuation losses that degrade both amplitude and resolution of seismic images at large depths.

These four parts were addressed by the first four tasks of the project. The following tasks addressed the practical application of the researched methodology on a real dataset acquired specifically for imaging ultra-deep structures, using very large offsets, 1,000 channels live (splitspread) onshore, and 500 channels live offshore (simulated split-spread) with 250 fold, a record length of 20 seconds, and maximum offsets of 41,200 feet (12,500 meters). The length of the record allowed us to image very deep structures and steep dips, while the large offsets offer good data redundancy and the ability to better discriminate the velocity of the deeper structures. One of the challenges of this project was to bring out this deeper information and image the geological structures deeper than 7-8 seconds, which was accomplished very successfully. The results on real data showed that ultra-deep wave-equation imaging provides much greater resolution and accuracy than what can be accomplished with standard imaging technology. This technology also opens for exploration areas with a very large discovery potential, like the Gulf of Mexico shelf, where reserves in the 250BCF range are being discovered at depths exceeding 15,000 feet, or the Gulf Coast onshore, which is now emerging as the next frontier to extend these plays. The onshore plays are expected to yield discoveries in the 1TCF range at depths between 20,000 and 30,000 ' and possibly 40,000'. The advanced imaging methodology may improve the success rate and cost effectiveness for new deep-field discoveries, and also has applications in increasing recovery efficiency for the development of existing fields. 


\section{Introduction}

Utilizing 3-D wave-equation migration for seismic imaging is a new approach that has shown great promise imaging the complex deep Gulf of Mexico structures. Previously, only Kirchhoff methods could be used to generate common-reflection point (CRP) gathers in offset domain and iteratively improve the velocity model used for imaging. Recent research has established a new approach to generate angle-domain common image gathers directly from 3-D wave-equation methods. The angle-gathers can be used to update the initial velocity model, and they form the basis for a novel method of 3-D migration velocity analysis. This technology can be used for oil and gas exploration in deep complex structures over 15,000 feet, where conventional single traveltime arrival Kirchhoff imaging (shown in Figure 1A) fails to provide an accurate structural image, while wave-equation imaging (shown in Figure 1B) provides much higher structural resolution and amplitude fidelity. This in turn allows the geophysicist to obtain higher resolution petrophysical information, linking the accurate seismic amplitude to reservoir properties like porosity, sand/shale content, water/oil saturation, $\mathrm{Vp} / \mathrm{Vs}$ ratio, etc. 3DGeo has been one of the pioneers in researching common image angle gathers technology and holds the patent (US6546339) for using the moveout of the angle gathers for computing the velocity update. The current work focused on wave-equation ultra-deep illumination and accurate amplitudes in conjunction with wave-equation imaging and velocity model building.

Due to a major gas shortage forecast for the U.S., oil and gas companies are increasing domestic exploration in an effort to find large gas reserves. One of the key areas of focus is the Gulf of Mexico shelf, where reserves in the $250 \mathrm{BCF}$ range are being discovered at depths exceeding 15,000 feet. Another key area, the gulf coast onshore, is now emerging as the next frontier to extend these plays. The onshore plays are expected to yield discoveries in the 1 TCF range at depths between 20,000 and 30,000' and possibly 40,000'. These plays are now referred to as ultra-deep. Ultra-deep plays present a significant opportunity for oil companies to add oil and gas reserves. The opportunity for seismic companies is also significant because legacy data sets whether proprietary or multi-client, fall short technically of what is required to open these new trends.
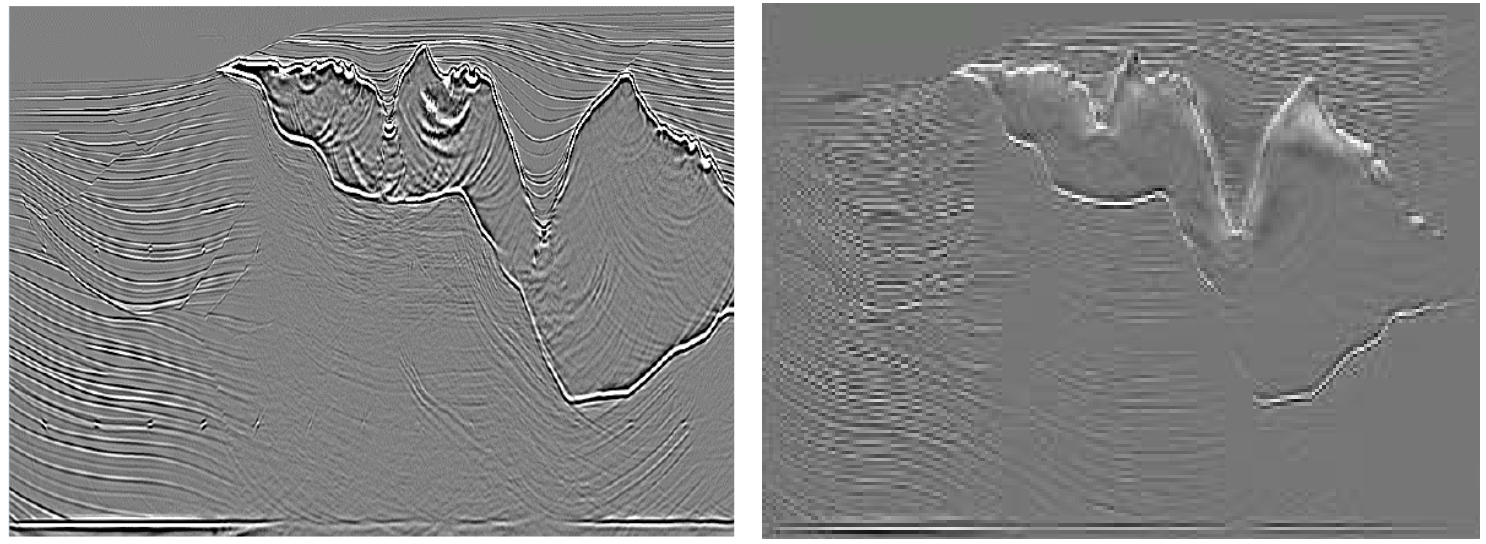

Figure 1: Comparison of standard Kirchhoff depth migration vs. wave-equation depth migration. A. The sediments under the salt body are not imaged correctly by Kirchhoff migration due to multi-pathing. B. Wave-equation depth migration focuses accurately the multiple arrivals under the salt. 
The U.S. Department of the Interior, Minerals Management Service OCS Report MMS 2001-036, assessed that the amount of Undiscovered, Conventionally Recoverable Resources in the deep Gulf of Mexico, is an average of 37 (high estimate of 45) billion barrels of oil, and 193 (high estimate of 207) trillion cubic feet of natural gas. At a conservative average price of $\$ 40$ per oil barrel and $\$ 8$ per MCF of gas, that is a value of $\$ 1480$ billion for the oil reserves and $\$ 1544$ billion for natural gas reserves. The importance of obtaining accurate images in these areas was highlighted by Kenneth J. Bayne, Deepwater Development Manager at Unocal (now Chevron): "In the Southern Green Canyon area, where fields such as Mad Dog, Holstein and Atlantis lie, the geology is less understood because some of the reservoirs are sub-salt and, thus, have lowerquality seismic." The lower-quality seismic refers to standard prestack depth imaging technology. Over the last 3-4 years the exploration industry has realized the importance of using wave-equation migration methods in parallel with Kirchhoff in the deep-water areas of the Gulf of Mexico, and 3DGeo has been part of the effort of using and demonstrating this technology.

True-amplitude imaging is necessary when the amplitudes of the seismic image are used as input for estimating the petrophysical properties of the reservoir rocks. Seismic imaging of deep targets requires a particular attention to amplitude preservation. Seismic signals are attenuated and scattered during propagation to deep targets. Compensating for the signal loss and for an irregular illumination of exploration targets at depth becomes a necessity both for obtaining a reliable structural image and for obtaining an image with meaningful amplitudes. In structurally complex media, both the single-arrival assumption and the classical asymptotic theory of Kirchhoff integrals become inadequate. Wave-equation imaging is identified a preferable alternative to the Kirchhoff method because of its ability to handle multiple arrivals, large velocity variations, and limited bandwidth wave-propagation effects. However, the theory of amplitude preservation in wave-equation imaging is less understood, and practical implementations still lack reliable tools of amplitude compensation. There are four parts of the amplitude compensation problem in wave-equation imaging:

5. Preserving true wave amplitudes at the downward wave-propagation step. True amplitudes are not automatically preserved by wave-equation methods, because the oneway equation, which serves as the basis for most of them, does not preserve the correct two-way equation amplitudes. Amplitude preservation at this step is especially important for imaging steeply dipping reflectors such as salt flanks and faults.

6. Recovering the true reflectivity (as a function of reflection angle) through applying the appropriate imaging condition. The overburden propagation effects should be removed at this step, recovering the true reflectivity at the image point.

7. Compensating for irregular target illumination resulting from incomplete seismic acquisition geometries. This step is crucial for imaging under salt bodies and in other difficult areas of deep exploration. Wave-equation methods (unlike ray-tracing methods) offer the additional benefit that the illumination can be studied for individual frequency bandwidths.

8. Compensating for non-elastic attenuation losses that degrade both amplitude and resolution of seismic images at large depths.

The following sections will describe in detail the mathematical and geophysical theory developed in this project and its application on synthetic and real data. 


\section{Ultra Deep Wave Equation Imaging and Illumination}

The goal of the project was to develop and test a novel technology designed to enhance seismic resolution and imaging of ultra-deep water complex geologic structures by using wave-equation depth migration, wave-equation velocity model building, and wave-equation illumination. The project was divided into several tasks that are listed below. The first group of tasks were centered around improving the theory and understanding of several aspects related to wave-propagation in deeper structures. The theory was then tested on synthetic data and finally applied on real data. The following tasks were executed during this project and will be described in detail in the following sections:

8. Confirm our ability to image true amplitude in wave-equation migration.

9. Implement alternative wave-equation amplitude imaging conditions.

10. Develop amplitude compensation for irregular illumination.

11. Investigate non-elastic attenuation and suggest approaches for recovery.

12. Demonstrate imaging and accuracy on POC-2 ultra-deep data.

13. Investigate effect of aperture on ultra-deep data.

14. Investigate the effect of large offsets on velocity discrimination on ultra-deep data.

15. Prepare Final Report.

\section{Task 1: Image true amplitude in wave-equation migration}

\section{True amplitude imaging theory}

The geometrical approach to wavefield continuation is based on the dynamic Huygens principle: consider each point $y$ on the surface $\partial D$ as an elementary point source and add contributions from all these sources, as follows:

$u^{( \pm)}(x, t)=\int_{\partial D} W(x, y) \hat{u}\left(y, t \mp T_{2}(x, y)\right) d y$

Here $u(x, t)$ is the wavefield inside the region $D$, while $\hat{u}(y, t)$ is the wavefield on the surface $\partial D$. Each elementary source is either delayed or advanced by the two-point traveltime $T_{2}(x, y)$ between $x$ and $y$ depending on whether the recorded wave exits or enters the domain. Additionally, each elementary contribution is assigned a weight $W(x, y)$. To understand why the geometrical construction (1) works, we need to develop two theoretical models: a geometrical model for the data $u(x, t)$ and a geometrical model for the effect of the integral operator (1).

We will focus our attention at some particular wavefront and assume that around that wavefront the wavefield can be represented as

$u(x, t) \approx A(x) f(t-T(x))$

where $A(x)$ is the geometrical amplitude, and $f(t)$ is a function with some singularity at $t=0$. Our task is to find how the three geometrical characteristics of the wavefield (functions $T, A$, and $f$ ) change in the wavefield continuation process. 


\section{Geometrical Integration}

With substitution of representation (2), equation (1) takes the form

$$
\begin{aligned}
u^{( \pm)}(x, t) & =\int_{\partial D} W(x, y) \hat{A}(y) f(t-T(y) \mp T(x, y)) d y \\
& =f(t) * \int_{\partial D} W(x, y) \hat{A}(y) \delta(t-T(y) \mp T(x, y)) d y
\end{aligned}
$$

where $*$ denotes a convolution operator, and $\delta(t)$ is the Dirac delta function defined through the property

$$
\int f(t) \delta(t) d t=f(0)
$$

\section{2-D integration}

Let us consider the 2-D case first, where $y$ is a one-dimensional variable. We will need to use the following property of the delta function: If function $\psi(t)$ has a single zero $t_{0}$ such that $\psi\left(t_{0}\right)=0$ and if $\psi(t)$ is invertible around $t_{0}$, then

$$
\int f(t) \delta(\psi(t)) d t=\int f\left(\psi^{-1}(\tau)\right) \delta(\tau)\left|\psi^{\prime}(t)\right|_{t=\psi^{-1}(\tau)}^{-1} d \tau=f\left(\psi^{-1}(0)\right)\left|\psi^{\prime}(t)\right|_{t=\psi^{-1}(0)}^{-1}=\frac{f\left(t_{0}\right)}{\left|\psi^{\prime}\left(t_{0}\right)\right|}
$$

Equation (5) suggests that a singularity in the integral in equation (3) occurs when the function $\psi(y)=t-\hat{T}(y) \pm T(x, y)$ has a zero partial derivative with respect to $y$. The condition

$$
\psi^{\prime}(y)=\frac{\partial}{\partial y}[\hat{T}(y) \mp T(x, y)]=0
$$

is precisely the familiar Fermat's principle for selecting the ray trajectory that goes from the surface to point $\mathrm{x}$ (Figure 1.1). Let us assume that Fermat's condition (6) is satisfied at some surface point $y=y_{0}$. The delta function in integral (3) is mostly affected by the neighborhood of $y_{0}$, where we can approximate the traveltime function by a quadratic

$t-\hat{T}(y) \pm T(x, y) \approx t-T^{( \pm)}(x) \pm \frac{1}{2} T_{y y}\left(y-y_{0}\right)^{2}$

where $T^{( \pm)}(x)=\hat{T}\left(y_{0}\right) \mp T\left(x, y_{0}\right)$ and $T_{y y}=\frac{\partial^{2} \hat{T}}{\partial y^{2}} \mp \frac{\partial^{2} T}{\partial y^{2}}$ evaluated at $y=y_{0}$. Equation (7) yields the following inverse expression for constraining the stationary point $y_{0}$ :

$\left|y=y_{0}\right|=\sqrt{\mp 2 \frac{t-T^{( \pm)}(x)}{T_{y y}}}$

provided that $T_{y y} \neq 0$ and that the expression under the square root is greater than zero. 


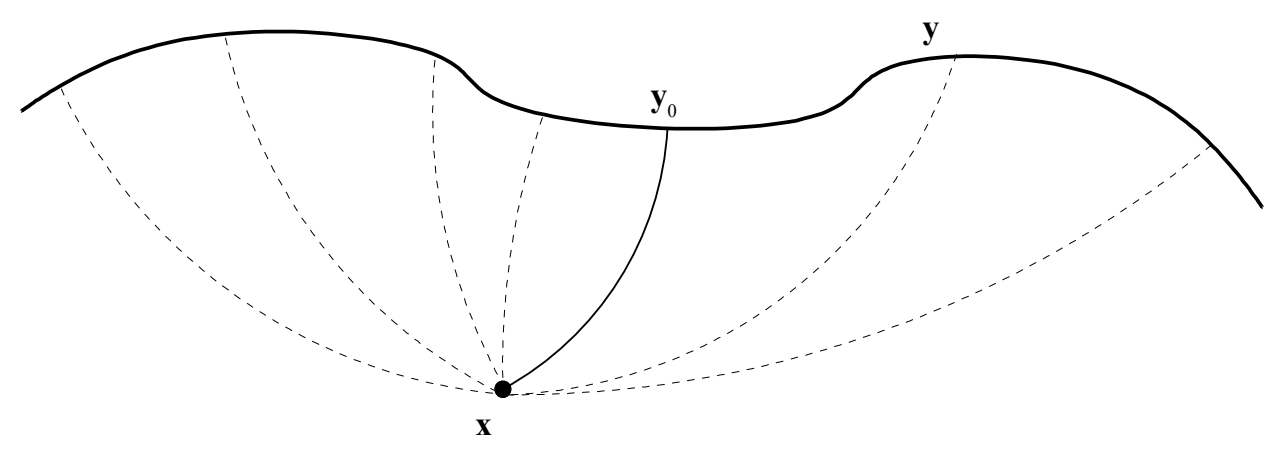

Figure 1.1: Geometrical wavefield extrapolation selects the effective ray trajectory according to Fermat's principle.

According to approximation (7) and equation (5), the effect of the integral operator in equation (3) can be described as

$$
\begin{gathered}
\int_{\partial D} W(x, y) \hat{A}(y) \delta(t-T(y) \mp T(x, y)) d y \approx \frac{W\left(x, y_{0}\right) \hat{A}\left(y_{0}\right)}{\left|T_{y y}\left(y-y_{0}\right)\right|} \\
=\frac{W\left(x, y_{0}\right) \hat{A}\left(y_{0}\right)}{\sqrt{2\left|T_{y y}\right|}} d_{1 / 2}\left[\mp \operatorname{sign}\left(T_{y y}\right)\left(t-T^{( \pm)}(x)\right)\right]
\end{gathered}
$$

where $d_{1 / 2}(t)$ is the discontinuous function

$$
d_{1 / 2}(t)=\left\{\begin{array}{c}
t^{-1 / 2} \text { for } \mathrm{t}>0 \\
0 \text { for } \mathrm{t} \leq 0
\end{array}\right.
$$

Comparing (9) with (2), we can see three effects of geometrical integration:

1. The wavefront discontinuity maps correctly from $t=T(y)$ to $t=T^{( \pm)}(x)$ with the effective ray trajectory selected according to Fermat's principle.

2. The amplitude gets multiplied by the $1 / \sqrt{\left|T_{y y}\right|}$ factor.

3. The waveform changes by convolution with $d_{1 / 2}(t)$. The form of $d_{1 / 2}(t)$ shows a discontinuity from the Fermat contribution followed by a tail of smaller-amplitude contributions from other points in the integration.

It is easy to compensate for the waveform distortion effect. Consider a family of signals

$$
r_{n}(t)=\left\{\begin{array}{cc}
\frac{t^{n}}{n !} & \text { for } t>0 \\
0 & \text { for } t \leq 0
\end{array}\right.
$$

defined for integer $n \geq 0$. Functions from this family have the property $d_{n}^{\prime}(t)=d_{n-1}(t)$. Formally, $d_{-1}(t)=d_{0}^{\prime}(t)=\delta(t)$, and $d_{n}(t)$ can be regarded as an impulse response of causal $(n+1)$-th order integration. The family is also formally extended to non-integer numbers $q$ with the help of the definition 
$r_{q}(t)=\left\{\begin{array}{c}\frac{t^{q}}{\Gamma(q+1)} \text { for } t>0 \\ 0 \text { for } t \leq 0\end{array}\right.$

where $\Gamma(t)$ is the special gamma function which has the property $\Gamma(n)=(n-1)$ ! for integer $n$ and $\Gamma(q+1)=q \Gamma(q)$ for any $q$ different from negative integers. With this definition, function $d_{1 / 2}(t)$ from equation (10) is proportional to the impulse response of half-order integration

$d_{1 / 2}(t)=\Gamma(1 / 2) r_{-1 / 2}(t)=\sqrt{\pi} r_{-1 / 2}(t)$

The inverse operation to convolution with $r_{-1 / 2}(t)$ is half-order differentiation or convolution with $r_{-3 / 2}(t)$. The waveform-compensated form of the 2-D wavefield continuation operator is therefore

$u^{( \pm)}(x, t)=D_{\mp \operatorname{sign}\left(T_{y y}\right)}^{1 / 2} \int_{\partial D} W(x, y) \hat{u}(y, t \pm T(x, y)) d y$

where $D_{\xi}^{1 / 2}$ denotes the operator of half-order differentiation with the causality defined by the sign of $\xi$. Figure 1.2 shows a numerical approximation of the half order derivative impulse response.

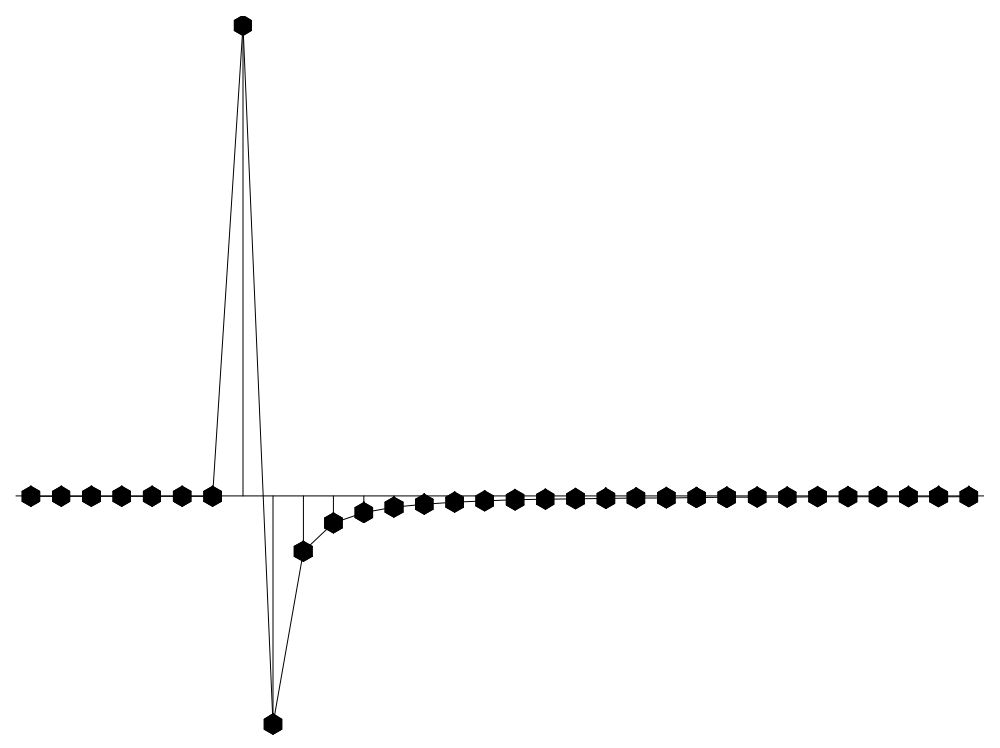

Figure 1.2: Numerical approximation of the half-order derivative impulse response. The approximation is computed in the Fourier transform domain by taking the square root of the first order finite-difference operator (Claerbout, 1993).

\section{3-D integration discussion}

What changes in the 3-D case? The $y$ variable becomes two-dimensional, and approximation (7) turns into 
$t-\hat{T}(y) \pm T(x, y) \approx t-T^{( \pm)}(x) \pm\left(y-y_{0}\right)^{T} \frac{1}{2} T_{y y}\left(y-y_{0}\right)$

where $T_{y y}=\frac{\partial^{2} \hat{T}}{\partial y^{2}} \mp \frac{\partial^{2} T}{\partial y^{2}}$ is the matrix of the second partial derivatives evaluated at $y=y_{0}$. If we put the origin of the y coordinate system at $y_{0}$, rotate the system to the principal (eigenvalue) directions of $T_{y y}$ and take the double integral in $d y$ sequentially, the following changes will occur with respect to the 2-D expression (9):

1. The value of $T_{y y}$ will be replaced with the product of the eigenvalues of $T_{y y}$ or, equivalently, the determinant of $T_{y y}$.

2. The half-order integration repeated twice will turn into full first-order integration. In summary, the corresponding 3-D expression is

$$
\int_{\partial D} W(x, y) \hat{A}(y) \delta(t-T(y) \mp T(x, y)) d y \approx \frac{W\left(x, y_{0}\right) \hat{A}\left(y_{0}\right)}{\sqrt{\left|\operatorname{det}\left(T_{y y}\right)\right|}} \frac{r_{1}\left[\mp \operatorname{sign}\left(T_{y y}\right)\left(t-T^{( \pm)}(x)\right)\right]}{2 \pi}
$$

In the 3-D case, compensation for the waveform distortion requires a full derivative operator $u^{( \pm)}(x, t)=D_{\mp \operatorname{sign}\left(T_{y y}\right)} \int_{\partial D} W(x, y) \hat{u}(y, t \pm T(x, y)) d y$

The only remaining undefined term in the wavefield extrapolation operators (15) and (18) is the amplitude weighting factor $W(x, y)$. We return to the question of defining $W$ and will derive a simple expression for it in a later section.

\section{FROM GEOMETRICAL TO WAVE EQUATION EXTRAPOLATION}

Starting from the geometrical representations (14) and (17), it is possible to derive a geometrically equivalent wave equation for wavefield extrapolation. Take the spatial gradient of the continued wavefield

$$
\nabla u^{( \pm)}=\int_{\partial D}\left[\nabla W \hat{A}(y) f(t-\hat{T}(y) \mp T(x, y)) \mp \nabla_{x} T W(x, y) \hat{A}(y) f^{\prime}(t-\hat{T}(y) \mp T(x, y))\right] d y
$$

Take the divergence of the gradient to obtain the Laplacian of the wavefield

$$
\begin{aligned}
\nabla^{2} u^{( \pm)} & =\int_{\partial D}\left[\nabla^{2} W \hat{A}(y) f(t-\hat{T}(y) \mp T(x, y))\right. \\
& \mp 2\left(\nabla W \cdot \nabla_{x} T\right) W(x, y) \hat{A}(y) f^{\prime}(t-\hat{T}(y) \mp T(x, y)) \\
& +\left(\nabla_{x} T \cdot \nabla_{x} T\right) W(x, y) \hat{A}(y) f^{\prime \prime}(t-\hat{T}(y) \mp T(x, y)) \\
& \left.\mp \nabla_{x}^{2} T W(x, y) \hat{A}(y) f^{\prime}(t-\hat{T}(y) \mp T(x, y))\right] d y
\end{aligned}
$$

In equation (19), the term $\left(\nabla_{x} T \cdot \nabla_{x} T\right)$ transforms to $S^{2}(x)$ according to the eikonal equation and can be taken out of the integral sign. The equation translates into 
$\nabla^{2} u=S^{2}(x) \frac{\partial^{2} u}{\partial t^{2}}+\cdots$

where ... stand for the terms that contain time derivatives of $f$ lower than the second order. If these terms are omitted, equation (20) turns into the classic wave equation. Note that, by squaring everything, we lost the \pm sign of wavefield extrapolation. Equation (20) describes both modes of wave propagation: waves entering into the domain and waves exiting the domain. If the required modes can be separated numerically, the wave equation can be used directly for wavefield continuation without the need to compute traveltimes explicitly. The power of the wave-equation approach becomes evident in the situation of multiple arrivals, when the two-point traveltime function $T(x, y)$ becomes multi-valued.

\section{GEOMETRICAL AMPLITUDES AND TRAVELTIME DERIVATIVES}

Geometrical spreading amplitude is a relative measure that describes amplitude changes due to changes in the propagating wavefront geometry. A well-known relationship connects the geometrical spreading and the determinant of the coordinate transformation matrix. The coordinate transformation is defined by ray tracing that maps the ray coordinates $\left\{\gamma_{1}, \gamma_{2}, T\right\}$ into Cartesian coordinates $x=\left\{x_{1}, x_{2}, x_{3}\right\}$. Here $\gamma_{1}$ and $\gamma_{2}$ are the coordinates that distinguish a ray in the family of rays from the source, and $T$ is traveltime along the ray. For fixed $\gamma_{1}$ and $\gamma_{2}, x(T)$ is the corresponding ray trajectory. One can define the geometrical spreading $J$ from the differential element of the wavefront area $d A=J d \gamma_{1} d \gamma_{2}$ and find its relationship to the coordinate transformation (Jacobian) matrix

$$
M=\left[\begin{array}{lll}
\frac{\partial x}{\partial \gamma_{1}} & \frac{\partial x}{\partial \gamma_{2}} & \frac{\partial x}{\partial T}
\end{array}\right]
$$

through the relationship

$$
\frac{J^{2}}{S^{2}}=\left|\frac{D(x)}{D\left(\gamma_{1}, \gamma_{2}, T\right)}\right|^{2}=\operatorname{det}\left(M^{T} M\right)=\operatorname{det}\left[\begin{array}{ccc}
E & F & 0 \\
F & G & 0 \\
0 & 0 & 1 / S^{2}
\end{array}\right]
$$

where $E=\frac{\partial x}{\partial \gamma_{1}} \cdot \frac{\partial x}{\partial \gamma_{1}}, G=\frac{\partial x}{\partial \gamma_{2}} \cdot \frac{\partial x}{\partial \gamma_{2}}, F=\frac{\partial x}{\partial \gamma_{1}} \cdot \frac{\partial x}{\partial \gamma_{2}}$, and $S$ is the slowness at point $x$.

Let us fix some point $x$ and orient the Cartesian coordinate system in such a way that $x_{3}$ is orthogonal to the wavefront, while the directions of $x_{1}$ and $x_{2}$ are tangential to the wavefront at $\mathrm{x}$. In this case, the geometrical spreading becomes related to the determinant of the 2-by-2 submatrix in $M$ :

$$
J=\left|\operatorname{det}\left[\begin{array}{ll}
\frac{\partial x_{1}}{\partial \gamma_{1}} & \frac{\partial x_{1}}{\partial \gamma_{2}} \\
\frac{\partial x_{2}}{\partial \gamma_{1}} & \frac{\partial x_{2}}{\partial \gamma_{2}}
\end{array}\right]\right|=\mid \operatorname{det}\left[\left.\begin{array}{ll}
\frac{\partial \gamma_{1}}{\partial x_{1}} & \frac{\partial \gamma_{1}}{\partial x_{2}} \\
\frac{\partial \gamma_{2}}{\partial x_{1}} & \frac{\partial \gamma_{2}}{\partial x_{2}}
\end{array}\right|^{-1}\right.
$$




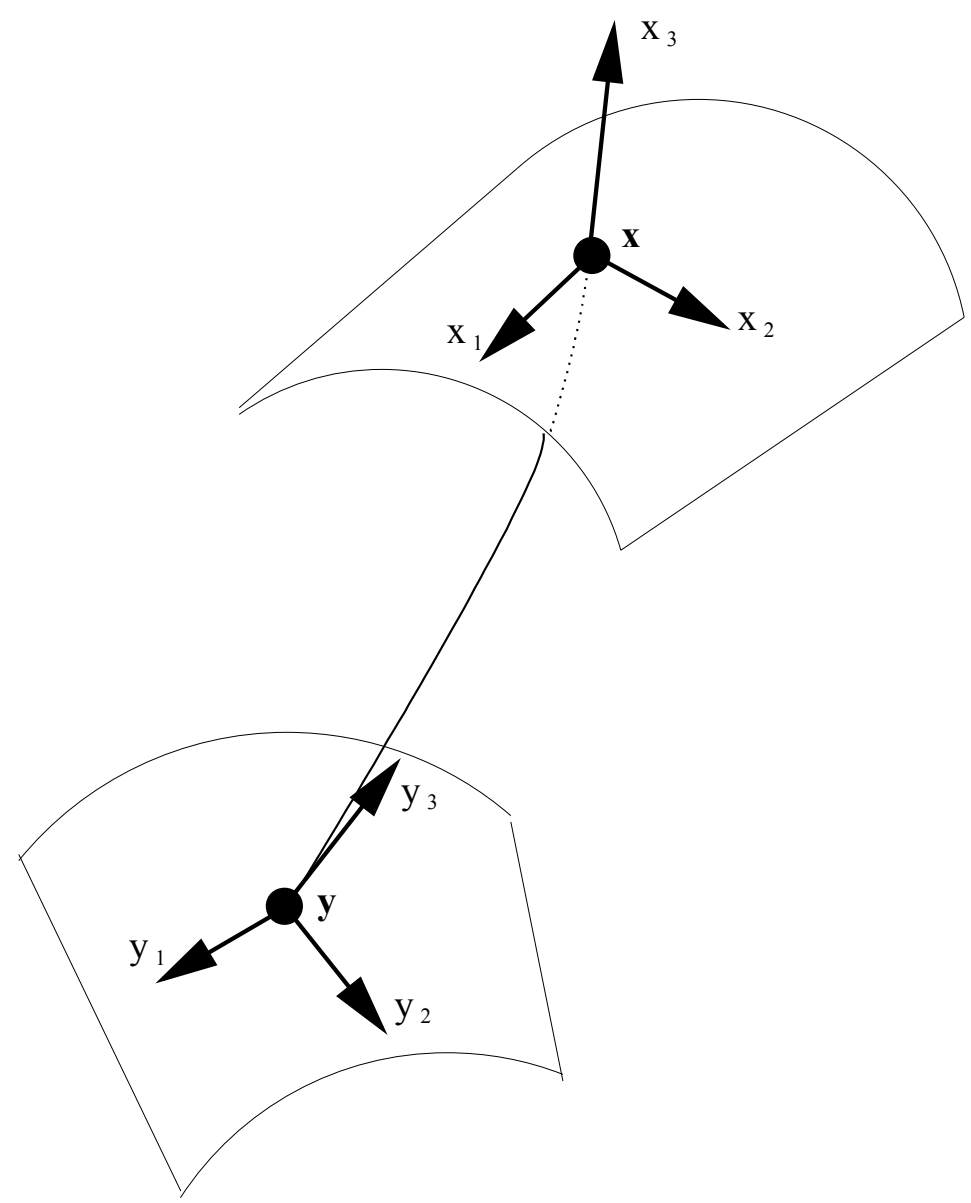

Figure 1.3: A coordinate system attached to a ray simplifies geometrical spreading derivations.

To define $\gamma_{1}$ and $\gamma_{2}$, we can now fix the ray source point $y$ and select the coordinate system for $y$ in such a way that $y_{3}$ is orthogonal to the initial source wavefront, while the directions of $y_{1}$ and $y_{2}$ are tangential to the source wavefront (Figure 1.3). The initial ray directions $\gamma_{1}$ and $\gamma_{2}$ can then be defined with the help of the first two coordinates $p_{1}$ and $p_{2}$ of the initial ray parameter vector $p$, as follows:

$\gamma_{1}=\frac{p_{1}}{S(y)}=\frac{1}{S(y)} \frac{\partial T}{\partial y_{1}}$
$\gamma_{2}=\frac{p_{2}}{S(y)}=\frac{1}{S(y)} \frac{\partial T}{\partial y_{2}}$

Substituting equations (24-25) into equation (23), we find a connection between the geometrical spreading and the mixed traveltime derivatives with respect to the source and receiver wavefront coordinates: 


$$
J(y, x)=S^{2}(y) \mid \operatorname{det}\left[\left.\begin{array}{ll}
\frac{\partial^{2} T}{\partial x_{1} \partial y_{1}} & \frac{\partial^{2} T}{\partial x_{2} \partial y_{1}} \\
\frac{\partial^{2} T}{\partial x_{1} \partial y_{2}} & \frac{\partial^{2} T}{\partial x_{2} \partial y_{2}}
\end{array}\right|^{-1}\right.
$$

It is not always convenient to explicitly rotate coordinate system at the source and the receiver for each individual ray. Let us imagine two arbitrary surfaces, one passing through $x$ and another passing through $y$. If $\hat{x}$ is the surface coordinate of $\mathrm{x}$, then we can relate vector $\left\{\partial T / \partial x_{1}, \partial T / \partial x_{2}\right\}$ to vector $\nabla_{\hat{x}} T$ through rotation by angle $\alpha_{x}$, which is the angle between the ray and the normal to the receiver surface. Similarly, $\left\{\partial T / \partial y_{1}, \partial T / \partial y_{2}\right\}$ is projected to $\nabla_{\hat{y}} T$ through rotation by $\alpha_{y}$. Using the surface coordinates $\hat{x}$ and $\hat{y}$, which are not necessarily aligned with the wavefronts, we can rewrite equation (26) in a more general form (Gritsenko, 1984)

$J(y, x)=S^{2}(y) \cos \alpha_{x} \cos \alpha_{y}\left|\operatorname{det}\left[\frac{\partial^{2} T}{\partial \hat{y} \partial \hat{x}}\right]\right|^{-1}$

where $\frac{\partial^{2} T}{\partial \hat{y} \partial \hat{x}}$ is the matrix of mixed second-order derivatives with respect to the source and receiver surface coordinates.

Equation (27) is especially convenient for describing geometric amplitude effects for wave propagation through layers between surfaces. We will use next for studying the amplitude behavior of seismic imaging operators.

\section{AMPLITUDES IN WAVEFIELD EXTRAPOLATION}

To describe the amplitude effects of wavefield extrapolation using equation (27), we need to consider three surfaces: the surface of the initial wave source $\hat{s}$, the surface of the recorded wavefield $\hat{y}$ and the surface to where we are continuing the wavefield $\hat{x}$. In this notation, the geometric wavefield extrapolation operator can be defined as

$u^{( \pm)}(x, t)=D_{t}^{( \pm)} \int W(x, y) \hat{u}(y, t \mp T(x, y)) d \hat{y}$

where $D_{t}^{( \pm)}$is the waveform correction operator, $W$ is the amplitude weight,

$T(x, y)$ is the traveltime between $x$ and $y, u^{( \pm)}(x, t)$ is the extrapolated wavefield (the \pm sign corresponds to forward and backward extrapolation) and is the wavefield recorded at the surface $\hat{y}$.

The output amplitude $A_{s x}$ is the product of three factors: the input amplitude $A_{s y}$ at the stationary point of integral (28), the weighting factor $W$, and the amplitude factor from geometrical integration, which is given by the stationary phase theory 


$$
F=\left|\operatorname{det}\left[\frac{\partial^{2} T_{s y}}{\partial \hat{y}^{2}} \pm \frac{\partial^{2} T_{y x}}{\partial \hat{y}^{2}}\right]\right|^{-1 / 2}
$$

Here we use a shortened notation, where $T_{i j}$ and $A_{i j}$ denote the traveltime and the amplitude between points $i$ and $j$ correspondingly.

The wave amplitude $A_{s y}$ is proportional, in geometrical approximation to $\sqrt{V(y) \rho(y) / J_{s y}}$, where $V(y)=1 / S(y)$ is velocity and $\rho(y)$ is density. Substituting equation (27),

$$
A_{s y}=V(s)\left|\frac{V(y) \rho(y)}{\cos \alpha_{s} \cos \alpha_{y}} \operatorname{det}\left[\frac{\partial^{2} T_{s y}}{\partial \hat{s} \partial \hat{y}}\right]\right|^{1 / 2}
$$

Analogously,

$$
A_{s x}=V(s)\left|\frac{V(x) \rho(x)}{\cos \alpha_{s} \cos \alpha_{x}} \operatorname{det}\left[\frac{\partial^{2} T_{s x}}{\partial \hat{s} \partial \hat{x}}\right]\right|^{1 / 2}
$$

In order to find the connection between the matrices of mixed second-order derivatives $\frac{\partial^{2} T_{s y}}{\partial \hat{s} \partial \hat{y}}$ and $\frac{\partial^{2} T_{s x}}{\partial \hat{s} \partial \hat{x}}$, let us write the traveltime relationship

$T_{s x}=T_{s y} \pm T_{y x}$

Fermat's principle or the condition of stationarity in integral (28) states that

$$
\frac{\partial T_{s y}}{\partial \hat{y}} \pm \frac{\partial T_{y x}}{\partial \hat{y}}=0
$$

Differentiating equation (32) with respect to $\hat{s}$,

$$
\frac{\partial T_{s x}}{\partial \hat{s}}=\frac{\partial T_{s y}}{\partial \hat{s}}+\left(\frac{\partial T_{s y}}{\partial \hat{y}} \pm \frac{\partial T_{y x}}{\partial \hat{y}}\right) \frac{\partial \hat{y}}{\partial \hat{s}}=\frac{\partial T_{s y}}{\partial \hat{s}}
$$

Differentiating equation (34) with respect to $\hat{x}$,

$$
\frac{\partial^{2} T_{s x}}{\partial \hat{s} \partial \hat{x}}=\frac{\partial^{2} T_{s y}}{\partial \hat{s} \partial \hat{y}} \frac{\partial \hat{y}}{\partial \hat{x}}
$$

The unknown derivative $\partial \hat{y} / \partial \hat{x}$ is found from Fermat's principle. Differentiating equation (33) with respect to $\hat{x}$,

$$
\left(\frac{\partial^{2} T_{s y}}{\partial \hat{y}^{2}} \pm \frac{\partial^{2} T_{y x}}{\partial \hat{y}^{2}}\right) \frac{\partial \hat{y}}{\partial \hat{x}} \pm \frac{\partial^{2} T_{y x}}{\partial \hat{y} \partial \hat{x}}=0
$$


Finally, expressing $\partial \hat{y} / \partial \hat{x}$ from equation (36) and substituting it into (35), we obtain the continuation formula for mixed traveltime derivatives (Blias et al, 1994)

$$
\frac{\partial^{2} T_{s x}}{\partial \hat{s} \partial \hat{x}}=\mp \frac{\partial^{2} T_{s y}}{\partial \hat{s} \partial \hat{y}}\left(\frac{\partial^{2} T_{s y}}{\partial \hat{y}^{2}} \pm \frac{\partial^{2} T_{y x}}{\partial \hat{y}^{2}}\right)^{-1} \frac{\partial^{2} T_{y x}}{\partial \hat{y} \partial \hat{x}}
$$

The inverted matrix is exactly the same one as in the stationary point factor (9) from the theory of geometrical integration. We can see that geometrical wavefield continuation supplies not only the correct geometrical transformation of wavefronts but also an amplitude factor needed for the correct transformation of amplitudes.

Finally, using the connection

$$
A_{s x}=A_{s y} W F
$$

and substituting equations (29-31) and (37), we can evaluate the appropriate weighting factor $W$ in integral (28), as follows (Goldin, 1987; 1991):

$$
W=\frac{A_{s x}}{A_{s y} F}=\left|\frac{V(x) \rho(x) \cos \alpha_{y}}{V(y) \rho(y) \cos \alpha_{x}} \operatorname{det}\left[\frac{\partial^{2} T_{y x}}{\partial \hat{y} \partial \hat{x}}\right]\right|^{1 / 2}=\frac{\cos \alpha_{y}}{V(y)} \frac{A_{y x}}{\sqrt{V(y) \rho(y)}}
$$

Remarkably, the weighting function depends only on the geometrical characteristics of wave propagation between $y$ and $x$ and not on the previous history of the wavefield.

\section{Building the benchmark model and benchmark dataset}

The velocity model in Figure 1.4 is the benchmark model for testing true amplitude wave equation imaging. It contains a shallow, gently dipping interface, as well as curved and steeply dipping interfaces. On the right side of the model the velocity variation is mostly depth dependent, while on the left side there is a strong velocity variation similar to the sharp velocity variation when a salt body is present. Therefore this benchmark will test gently dipping interfaces, curved interfaces, and steeply dipping interfaces in the presence of depth variable velocity as well as rapidly varying velocity. 


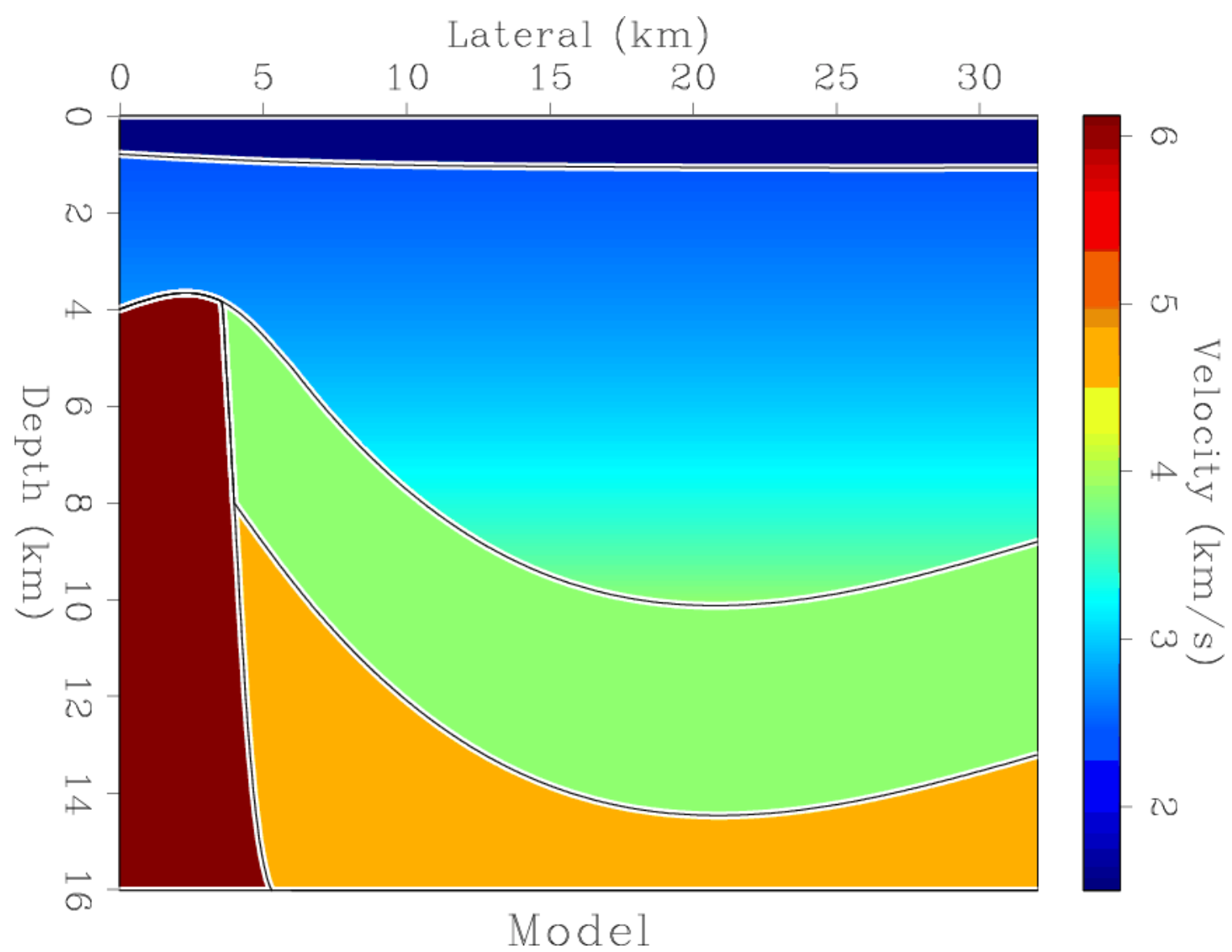

Figure 1.4 Benchmark velocity model for testing true amplitude wave equation imaging.

We ran a finite difference wave-equation shot profile modeling over this model to build a waveequation amplitude consistent synthetic model that would be imaged with different amplitude and imaging schemes. There were 1280 shots used to create the synthetic dataset we used to benchmark true amplitude migration. The grid spacing is $10 \times 10$ meters to avoid numerical dispersion. Figures 1.5A-C show several snapshots of wave equation propagation through the benchmark velocity model. Figure 1.6 shows two constant offset sections through the benchmark dataset. Figure 1.7 shows two wave-equation migrations of the synthetic dataset, using only $10 \%$ of the available shots, using no amplitude term and using a new amplitude compensation term. The effects of the new amplitude term can already be seen on the deeper events and the steeply dipping events, which appear imaged with more energy. 


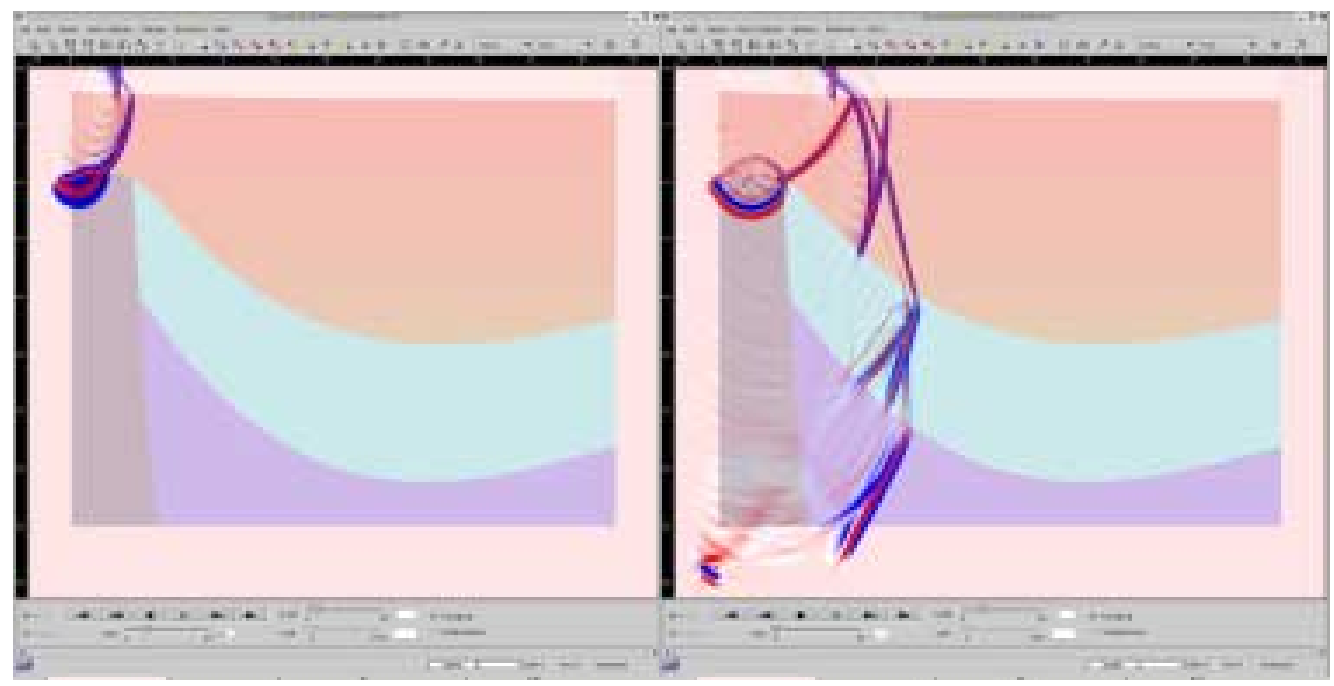

Figure 1.5A: Wave propagation through the benchmark velocity model.

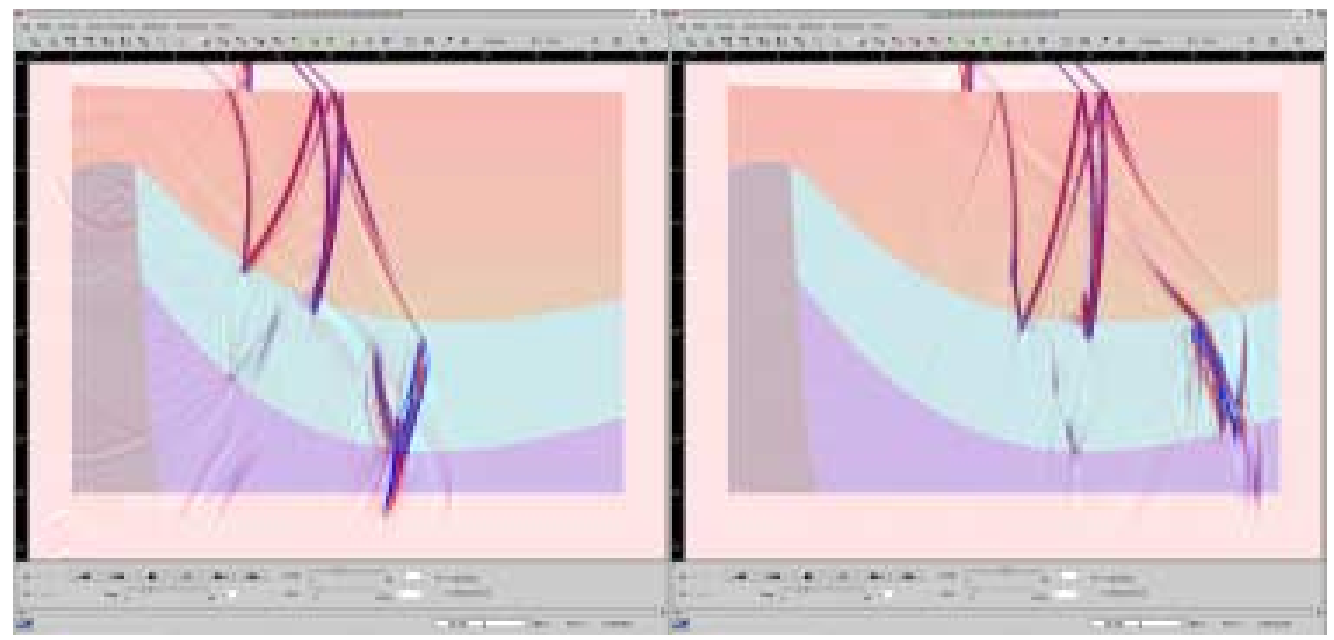

Figure 1.5B: Wave propagation through the benchmark velocity model.

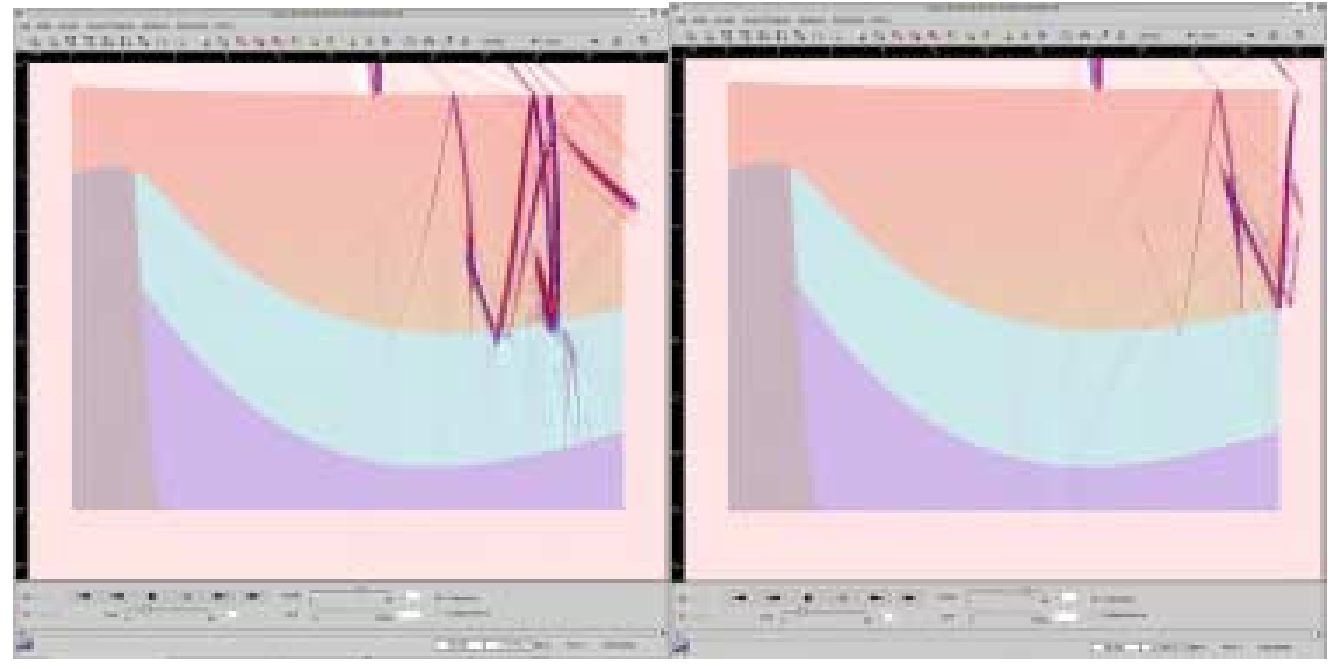

Figure 1.5C: Wave propagation through the benchmark velocity model. 

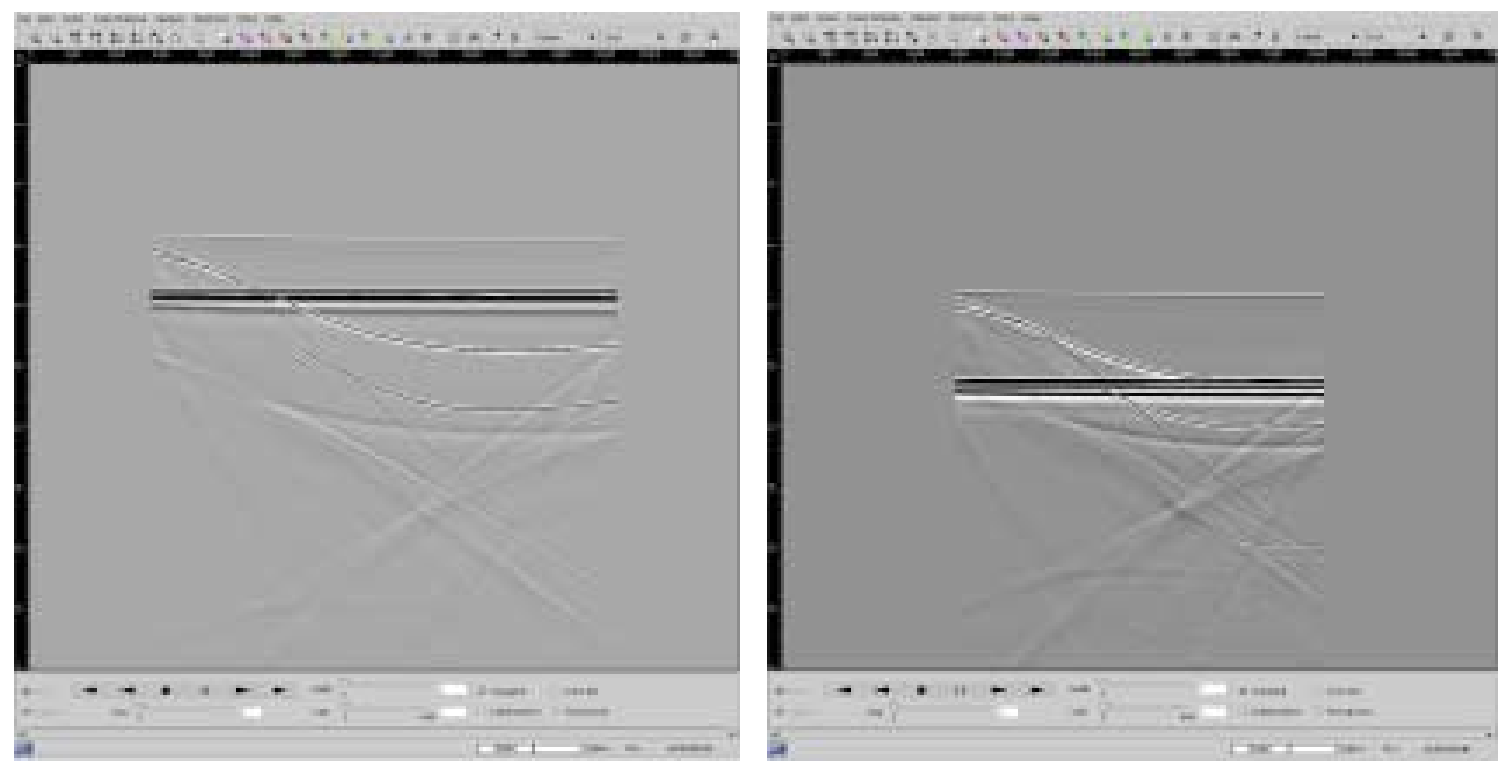

Figure 1.6: Constant offset sections from the synthetic dataset.
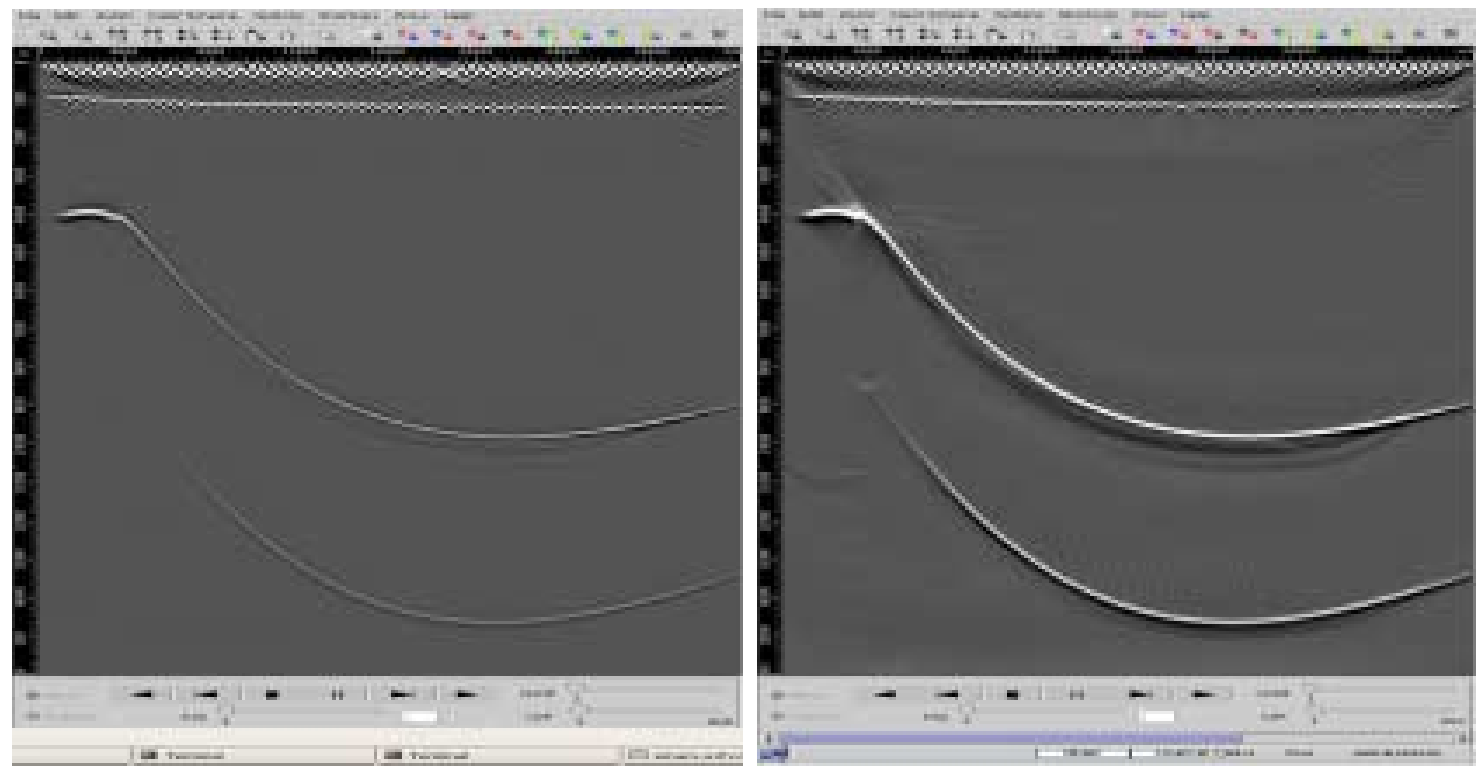

Figure 1.7: Shot profile migration of the synthetic dataset. Left: No amplitude term. Right: New amplitude term. Deep events and dipping events are boosted.

\section{AMPLITUDES IN KIRCHHOFF AND WAVE-EQUATION MIGRATION}

The definition of common-shot migration simply follows from a backward continuation operator and the application of the imaging condition. To construct the imaging operator, we need to take the output of wavefield continuation (28) for $u^{(-)}$and evaluate it at the time corresponding to the forward propagation from the source. The result is

$$
I(x ; s)=\int W_{M}(x, s, r) D_{t}^{(-)} u(s, r, T(s, x)+T(x, r)) d \hat{r}
$$


The migration operator (40) transforms the reflection data $u(s, r, t)$ for the source $s$, receiver $r$, and time $t$, to the image $I(x ; s)$ defined in space $x$ for every source $s$. If we use the wavefield extrapolation weight (39) as the migration weight $W_{M}$ in equation (40), the imaged reflectors will acquire the amplitude of the wavefield at the time of reflection $A_{s x}$. To remove this effect and reveal only the amplitude reflectivity coefficient, we need to divide the amplitude by $A_{s x}$. The true-amplitude common-shot migration weight is therefore (Keho and Beydoun, 1988; Goldin, 1992; Tygel et al., 1994)

$W_{M}=\frac{W}{A_{s x}}=\frac{\cos \alpha_{y}}{V(y)} \frac{A_{y x}}{A_{s x}} \frac{1}{\sqrt{V(y) \rho(y)}}$

In the case of the wave-equation migration, this construction is analogous to the division of upgoing and downgoing wavefields (Claerbout, 1970).

Figure 1.7 shows the differences between applying the correct amplitude terms and not applying them. Figure 1.8 shows the effect in the angle direction. Figure 1.9 shows the amplitude term effect variation with angle, by comparing the output of the theoretical reflection coefficient variation with angle calculated using the Zoeppritz equation with the output of the wave-equation migration angle gather. The amplitude variation with angle is consistent with the Zoeppritz equation.
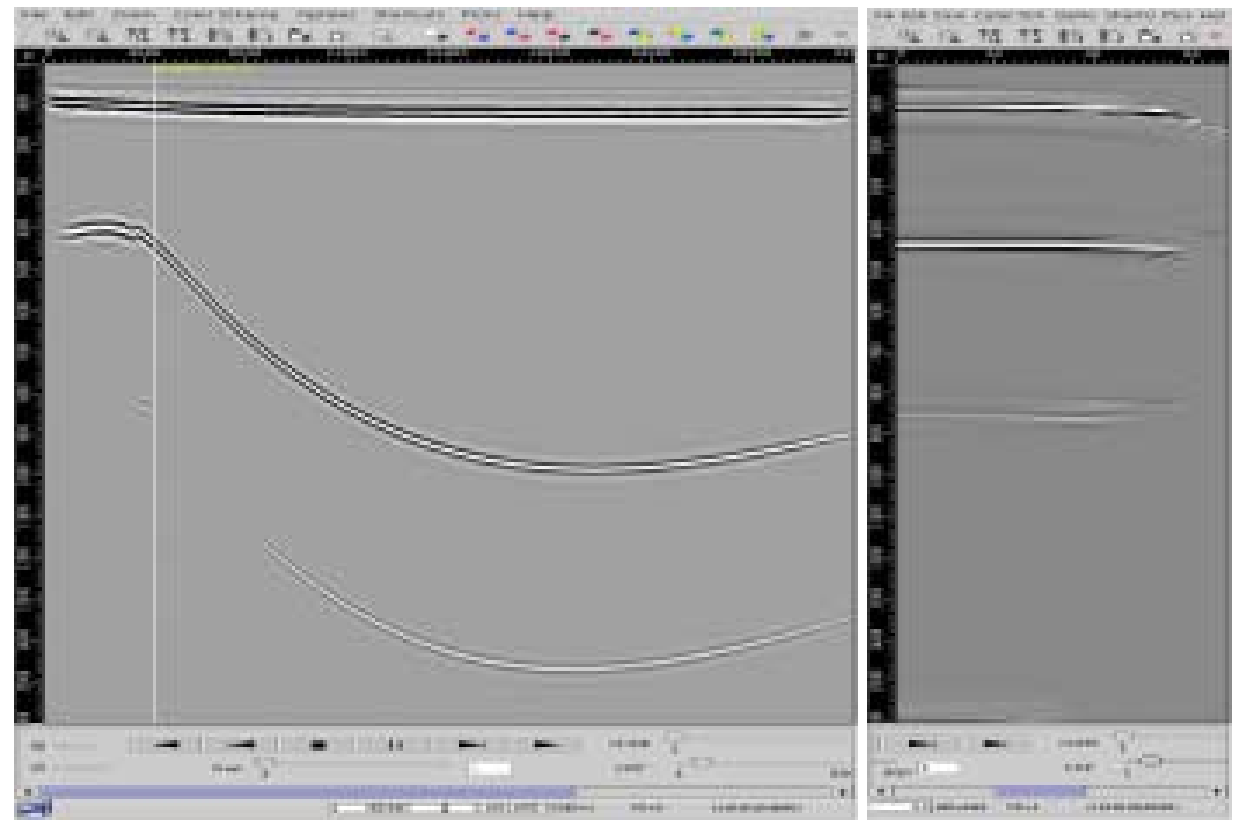

Figure 1.8: Shot profile migration of the synthetic dataset. Left: Stacked image. Right: Angle gather at the location of the thin white line. 

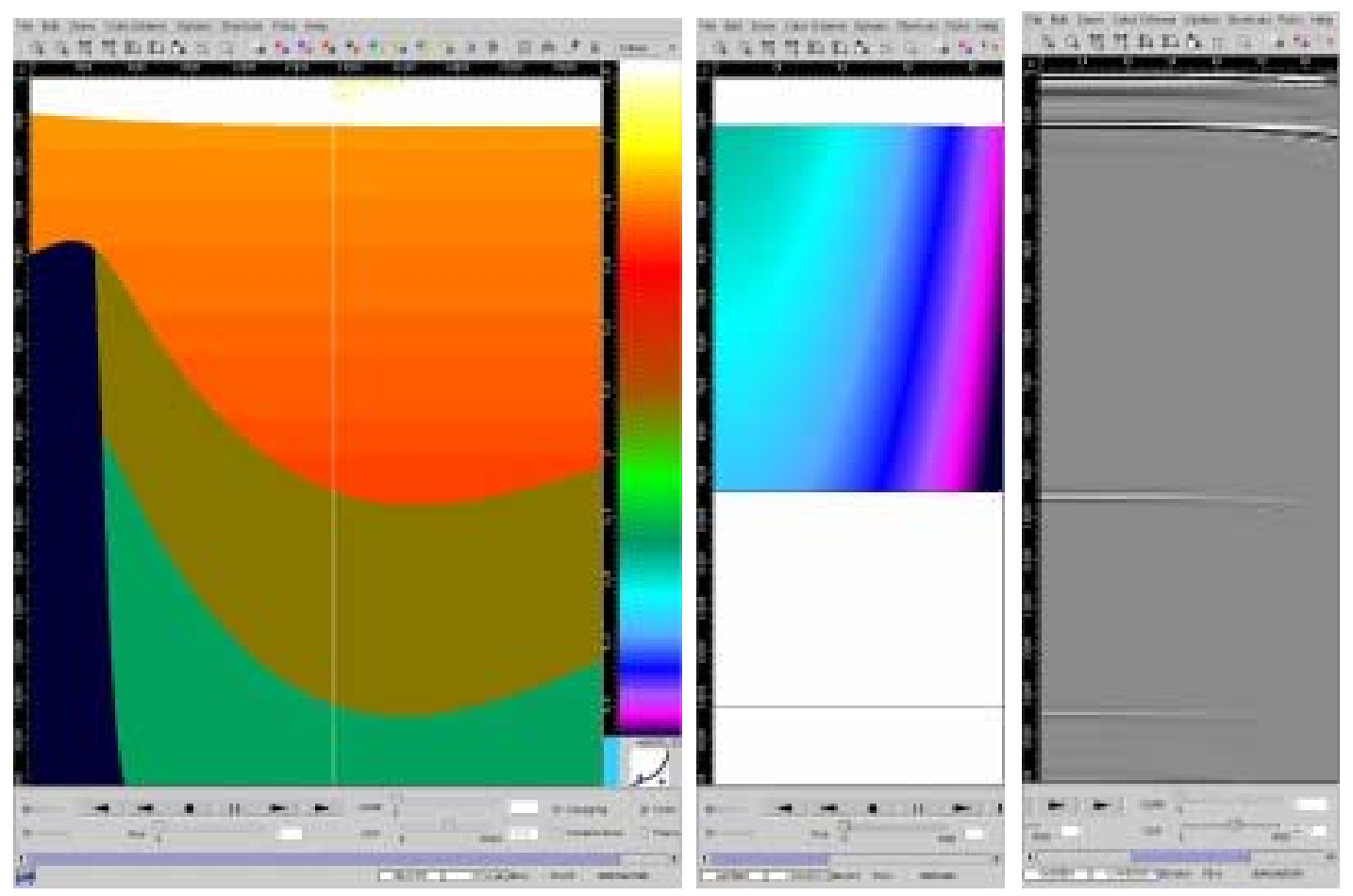

Figure 1.9: Left: Benchmark velocity model with the location of the angle gather. Middle: Reflection coefficient amplitude with angle calculated using the Zoeppritz equation. Right: Angle gather at the location of the thin white line. The amplitude variation with angle is consistent with the Zoeppritz equation.

\section{Task 2: Implement an alternative wave-equation amplitude imaging condition}

We derive a new generalized imaging condition based on time shifts between source and receiver wavefields. This imaging condition contrasts with other imaging techniques requiring space shifts between the two wavefields. This imaging condition is applicable to both Kirchhoff and wave-equation migrations (Sava and Fomel, 2005). The transformation allows us to generate common-image gathers presented as a function of either time-shift or pseudo-angle at every location in space. Inaccurate migration velocity is revealed by common-image gathers with nonflat events.

A key challenge for imaging in complex areas is accurate determination of a velocity model that describes with sufficient precision wave propagation in the area under investigation. Migration velocity analysis is based on image accuracy indicators that are optimized when data are correctly imaged. A common procedure for velocity analysis is based on alignment of images created with multi-offset data. An optimal choice of image analysis can be done in the angle domain which is free of some of the complicated artifacts present in offset gathers in complex areas (Stolk and Symes, 2002).

A key component of such image decompositions is the imaging condition. A careful implementation of this imaging condition preserves all information necessary to decompose images in their angle-dependent components. The challenge is not only to use these angledependent images for velocity or amplitude analysis, but also to construct them cheaply, reliably and with direct access to velocity information. 


\section{Conventional imaging condition}

A conventional imaging condition for shot-record migration, often referred-to as UD imaging condition (Claerbout, 1985), consists of time cross-correlation at every image location between the source and receiver wavefields, followed by image extraction at zero time:

$$
\begin{aligned}
& U(\mathbf{m}, t)=U_{r}(\mathbf{m}, t) \otimes U_{s}(\mathbf{m}, t), \\
& R(\mathbf{m})=U(\mathbf{m}, t=0),
\end{aligned}
$$

where the symbol $\otimes$ denotes cross-correlation in time. Here, $\mathbf{m}=[\mathrm{x}, \mathrm{y}, \mathrm{z}]$ is a vector describing the locations of image points, $U_{s}(\mathbf{m}, \mathrm{t})$ and $U_{r}(\mathbf{m}, \mathrm{t})$ are source and receiver wavefields respectively, and $R(\mathbf{m})$ denotes a migrated image. A final image is obtained by summation over shots.

For computational reasons, this imaging condition is usually implemented in the Fourier domain using the expression

$$
R(\mathbf{m})=\sum_{\omega} U_{r}(\mathbf{m}, \omega) U_{s}^{*}(\mathbf{m}, \omega)
$$

The * sign represents a complex conjugate applied on the receiver wavefield $U_{\mathrm{s}}$ in the Fourier domain

\section{Space-shift imaging condition}

A generalized prestack imaging condition (Sava and Fomel, 2005) estimates image reflectivity using cross-correlation in space and time, followed by image extraction at zero time:

$$
\begin{aligned}
& U(\mathbf{m}, \mathbf{h}, t)=U_{r}(\mathbf{m}+\mathbf{h}, t) \otimes U_{s}(\mathbf{m}-\mathbf{h}, t), \\
& R(\mathbf{m}, \mathbf{h})=U(\mathbf{m}, \mathbf{h}, t=0) .
\end{aligned}
$$

Here, $\mathbf{h}=\left[\mathrm{h}_{\mathrm{x}}, \mathrm{h}_{\mathrm{y}}, \mathrm{h}_{\mathrm{z}}\right]$ is a vector describing the local source-receiver separation in the image space. Special cases of this imaging condition are horizontal space-shift (Rickett and Sava, 2002) and vertical space-shift (Biondi and Symes, 2004).

As for the conventional imaging condition, this imaging condition can be implemented in the Fourier domain using the expression

$$
R(\mathbf{m}, \mathbf{h})=\sum_{\omega} U_{r}(\mathbf{m}+\mathbf{h}, \omega) U_{s}^{*}(\mathbf{m}-\mathbf{h}, \omega)
$$

\section{Time-shift imaging condition}

Another prestack imaging condition involves shifting of the source and receiver wavefields in time, as opposed to space, followed by image extraction at zero time:

$$
\begin{aligned}
& U(\mathbf{m}, \tau, t)=U_{r}(\mathbf{m}, t+\tau) \otimes U_{s}(\mathbf{m}, t-\tau), \\
& R(\mathbf{m}, \tau)=U(\mathbf{m}, \tau, t=0) .
\end{aligned}
$$

Here, $\tau$ is a time shift between the source and receiver wavefields prior to imaging. This imaging condition can be implemented in the Fourier domain using the expression

$$
R(\mathbf{m}, \tau)=\sum_{\omega} U_{r}(\mathbf{m}, \omega) U_{s}^{*}(\mathbf{m}, \omega) e^{2 i \omega \tau}
$$

which simply involves a phase-shift applied to the wavefields prior to summation over frequency $\omega$ for imaging at zero time. 


\section{ANGLE TRANSFORMATION IN WAVE-EQUATION IMAGING}

Using the definitions introduced in the preceding section, we can make the standard notations for source and receiver coordinates: $\mathbf{s}=\mathbf{m}-\mathbf{h}$ and $\mathbf{r}=\mathbf{m}+\mathbf{h}$. The traveltime from a source to a receiver is a function of all spatial coordinates of the seismic experiment $t=t(\mathbf{m}, \mathbf{h})$. Differentiating $t$ with respect to all components of the vectors $\mathbf{m}$ and $\mathbf{h}$, and using the standard notations

$$
\mathbf{p}_{\alpha}=\nabla_{\alpha} t
$$

where $\boldsymbol{\alpha}=\{\mathbf{m}, \mathbf{h}, \mathbf{s}, \mathbf{r}\}$, we can write:

$$
\begin{aligned}
& 2 \mathbf{p}_{\mathrm{m}}=\mathbf{p}_{\mathrm{r}}+\mathbf{p}_{\mathrm{s}} \\
& 2 \mathbf{p}_{\mathrm{h}}=\mathbf{p}_{\mathrm{r}}-\mathbf{p}_{\mathrm{s}}
\end{aligned}
$$

From equations (11)-(12), we can write

$$
\begin{aligned}
& \mathbf{p}_{\mathrm{s}}=\mathbf{p}_{\mathrm{m}}-\mathbf{p}_{\mathrm{h}}, \\
& \mathbf{p}_{\mathrm{r}}=\mathbf{p}_{\mathrm{m}}+\mathbf{p}_{\mathrm{h}} .
\end{aligned}
$$

By analyzing the geometric relations of various vectors at an image point (Figure 2.1), we can write the following trigonometric expressions:

$$
\begin{aligned}
& 4\left|\mathbf{p}_{\mathbf{h}}\right|^{2}=\left|\mathbf{p}_{\mathbf{s}}\right|^{2}+\left|\mathbf{p}_{\mathbf{r}}\right|^{2}-2\left|\mathbf{p}_{\mathbf{s}} \| \mathbf{p}_{\mathbf{r}}\right| \cos (2 \theta), \\
& 4\left|\mathbf{p}_{\mathbf{h}}\right|^{2}=\left|\mathbf{p}_{\mathbf{s}}\right|^{2}+\left|\mathbf{p}_{\mathbf{r}}\right|^{2}-2\left|\mathbf{p}_{\mathbf{s}} \| \mathbf{p}_{\mathbf{r}}\right| \cos (2 \theta) .
\end{aligned}
$$

Figure 2.1: Geometric relations between ray vectors at a reflection point.

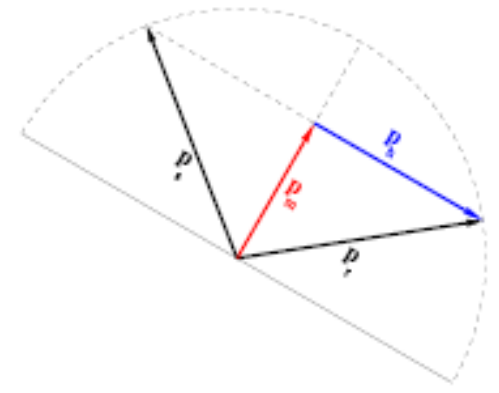

Equations (15)-(16) relate wavefield quantities, $\mathbf{p}_{\mathrm{h}}$ and $\mathbf{p}_{\mathrm{m}}$, to geometric quantities, reflection angle $\theta$. Analysis of these expressions provide sufficient information for complete decompositions of migrated images in components for different reflection angles.

\section{Space-shift imaging condition}

Defining $\mathbf{k}_{\mathrm{m}}$ and $\mathbf{k}_{\mathrm{h}}$ as location and offset wavenumber vectors, and assuming $|\mathbf{p s}|=|\mathbf{p r}|=s$, where $s$ (m) is the slowness at image locations, we can replace $|\mathbf{p m}|=|\mathbf{k m}| / \omega$ and $|\mathbf{p h}|=|\mathbf{k h}| / \omega$ in equations (15)-(16):

$$
\begin{aligned}
& 4\left|\mathbf{p}_{\mathbf{h}}\right|^{2}=2(\omega s)^{2}(1-\cos 2 \theta), \\
& 4\left|\mathbf{p}_{m}\right|^{2}=2(\omega s)^{2}(1+\cos 2 \theta) .
\end{aligned}
$$

Using the trigonometric identity

$$
\cos 2 \theta=\frac{1-\tan ^{2} \theta}{1+\tan ^{2} \theta}
$$

we can eliminate from equations (17)-(18) the dependence on frequency and slowness, and obtain an angle decomposition formulation after imaging by expressing $\tan \theta$ function of position and offset wavenumbers $(\mathbf{k m}, \mathbf{k h})$ : 


$$
\tan \theta=\frac{\left|\mathbf{k}_{\mathbf{h}}\right|}{\left|\mathbf{k}_{\mathbf{m}}\right|} .
$$

We can construct angle-domain common-image gathers by transforming prestack migrated images using equation (20)

$$
R(\mathbf{m}, \mathbf{h}) \Rightarrow R(\mathbf{m}, \theta)
$$

In $2 \mathrm{D}$, this transformation is equivalent with a slant-stack on migrated offset gathers. For $3 \mathrm{D}$, this transformation is described more detail by Fomel (2004) or Sava and Fomel (2005).

\section{Time-shift imaging condition}

Using the same definitions as the ones introduced in the preceding subsection, we can rewrite equation (18) as

$$
\left|\mathbf{p}_{\mathbf{m}}\right|^{2}=s^{2} \cos ^{2} \theta
$$

from which we can derive an expression for angle-transformation after time-shift prestack imaging:

$$
\cos \theta=\frac{\left|\mathbf{p}_{\mathbf{m}}\right|}{s}
$$

Relation (23) can be interpreted using ray parameter vectors at image locations (Figure 2.2). Angle-domain common-image gathers can be obtained by transforming prestack migrated images using equation (23):

$$
R(\mathbf{m}, \tau) \Rightarrow R(\mathbf{m}, \theta)
$$

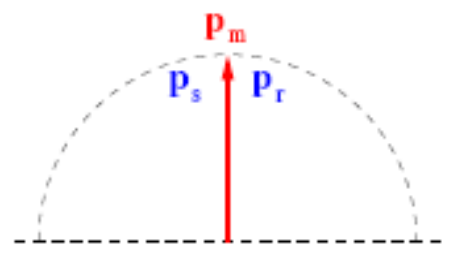

(a)

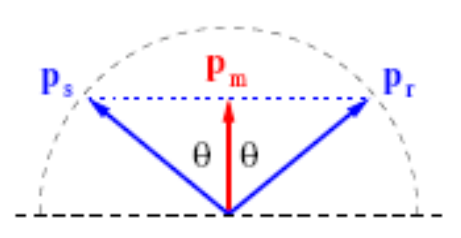

(b)

Figure 2.2: Interpretation of angle-decomposition based on equation (23) for time-shift gathers.

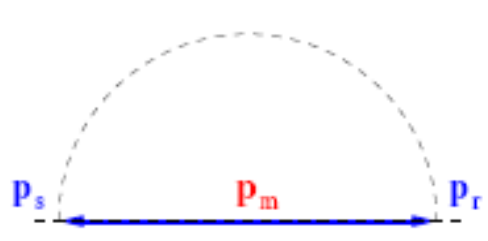

(c) 
Equation (23) can be written as

$$
\cos ^{2} \theta=\frac{\left|\nabla_{\mathbf{m}} \tau\right|^{2}}{s^{2}(\mathbf{m})}=\frac{\tau_{x}^{2}+\tau_{y}^{2}+\tau_{z}^{2}}{s^{2}(x, y, z)},
$$

where $\tau_{x}, \tau_{y}, \tau_{z}$ are partial derivatives of $\tau$ relative to $x, y, z$. We can rewrite equation (25) as

$$
\cos ^{2} \theta=\frac{\tau_{z}^{2}}{s^{2}(x, y, z)}\left(1+z_{x}^{2}+z_{y}^{2}\right)
$$

where $z_{x}$ and $z_{y}$ denote partial derivative of coordinate $z$ relative to coordinates $x$ and $y$, respectively. Equation (26) describes an algorithm in two steps for angle-decomposition after time-shift imaging: compute $\cos \theta$ through a slant-stack in $z-\tau$ panels, then apply a correction using the migration slowness $s$ and a function of the structural dip $\sqrt{1+z_{x}^{2}+z_{y}^{2}}$.

\section{EXAMPLES}

Figures 2.3-2.5 show examples of imaging using space-shift and time-shift imaging conditions of the Sigsbee2A synthetic model (Paffenholtz et at., 2002).

Figure 2.3 shows the velocity model (top) and an image obtained by shot-record migration of all shots.
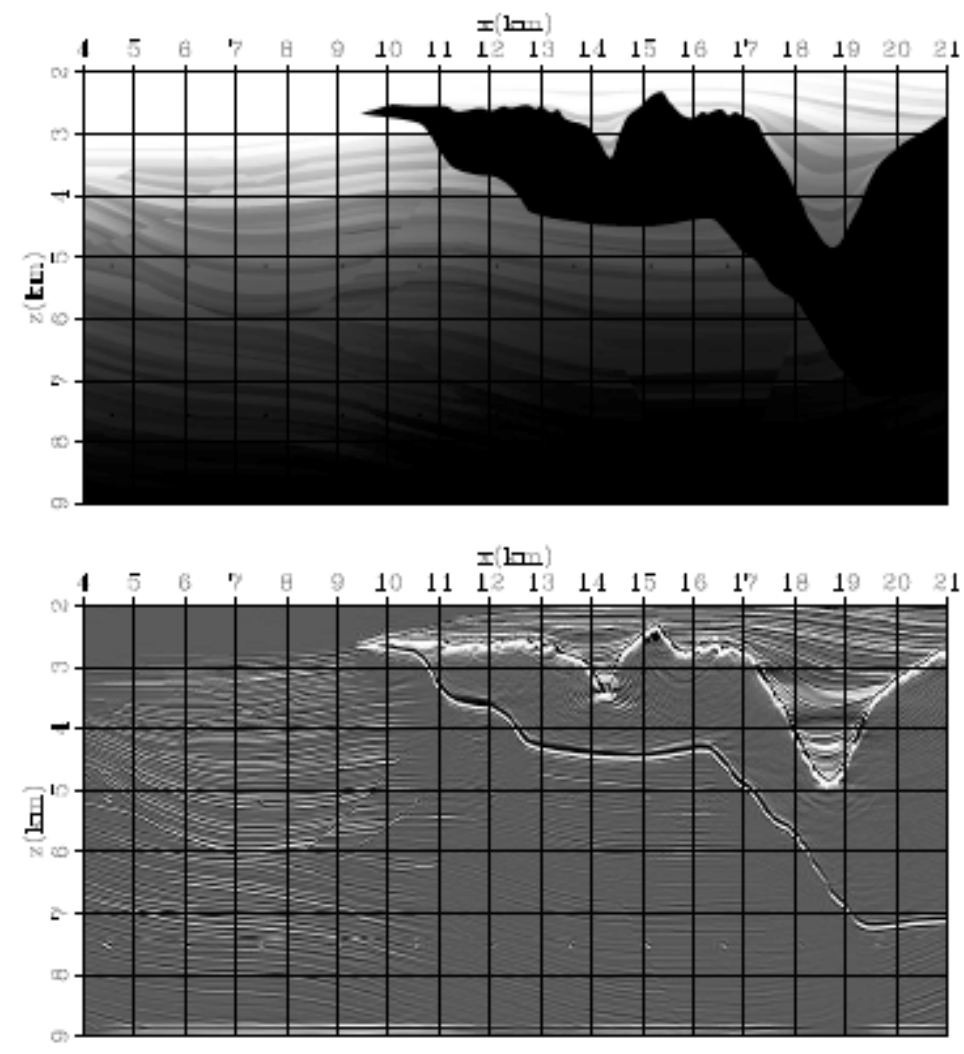

Figure 2.3: Sigsbee 2A model: correct velocity (top) and migrated image obtained by shot-record wavefield extrapolation migration with time-shift imaging (bottom). 
Figure 2.4 shows one common-image gather obtained by space-shift imaging at $\mathrm{x}=7 \mathrm{~km}$. From left to right, the panels depict: the image gather, the gather after slant-stack, and the gather after conversion to reflection angle.

Figure 2.4: Space-shift imaging: offset-gather (left), slant-stacked gather (middle) and anglegather (right).
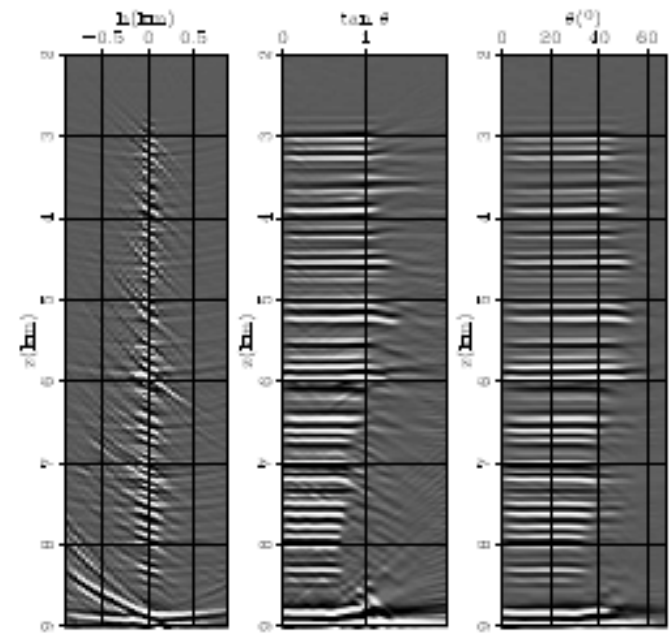

Figure 2.5 shows one common-image gather obtained by time-shift imaging at $x=7 \mathrm{~km}$. From left to right, the panels depict: the image gather, the gather after slant-stack, and the gather after conversion to reflection angle.

Figure 2.5: Time-shift imaging: offset-gather (left), slant-stacked gather (middle) and anglegather (right).
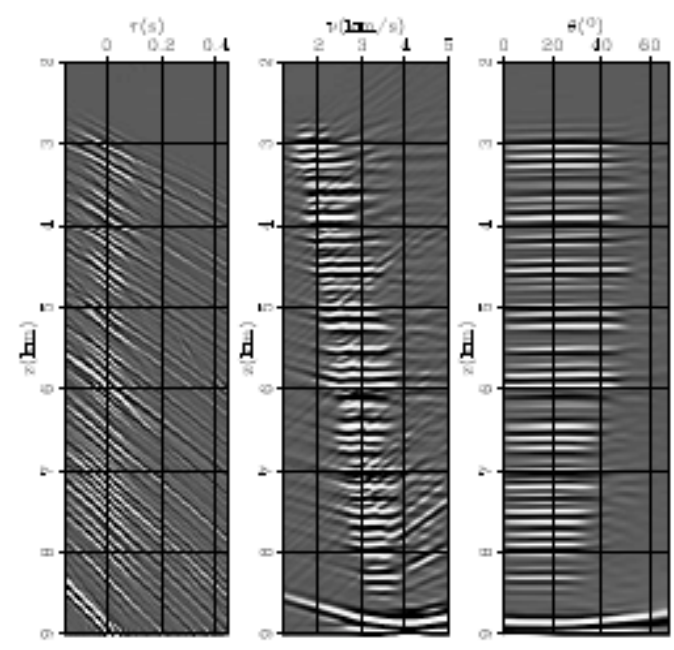

Task 3: Develop amplitude compensation for irregular illumination

Seismic signals are attenuated and scattered during propagation to deep targets. Compensating for the signal loss and for an irregular illumination of exploration targets at depth becomes a necessity both for obtaining a reliable structural image and for obtaining an image with meaningful amplitudes. This is an important area of research in modern depth imaging, and it has not been fully investigated in the industry. The current work tries to understand the illumination effects and their frequency variation, and the effect of velocity model in focusing and defocusing 
the energy propagation through the earth. Several other aspects like the dip of the structural model and the angle dependence of illumination in a particular acquisition geometry need to be addressed in the future. This will provide a better mechanism to understand and compensate for the effects of illumination to the transmission of the seismic energy through the earth model and their impact on the imaging amplitudes.

Using the velocity model developed part of Task 5, we performed several illumination tests to examine the variation in amplitude with shot location and frequency range. Figure 3.1 shows the illumination using a $5 \mathrm{~Hz}$ source while Figure 3.2 shows the illumination using $10 \mathrm{~Hz}$, and Figure 3.3 shows the illumination using $20 \mathrm{~Hz}$. The differences are not as pronounced as in the case of fast lateral velocity variations, for instance in the presence of salt. The illumination effects were compensated in the imaging algorithm. Since the velocity model has mild lateral velocity variations, we found the illumination effects in this model to be mild, not as dramatic as in the presence of salt bodies. Figure 3.4 shows the illumination using a $5 \mathrm{~Hz}$ source superimposed on the velocity model. 


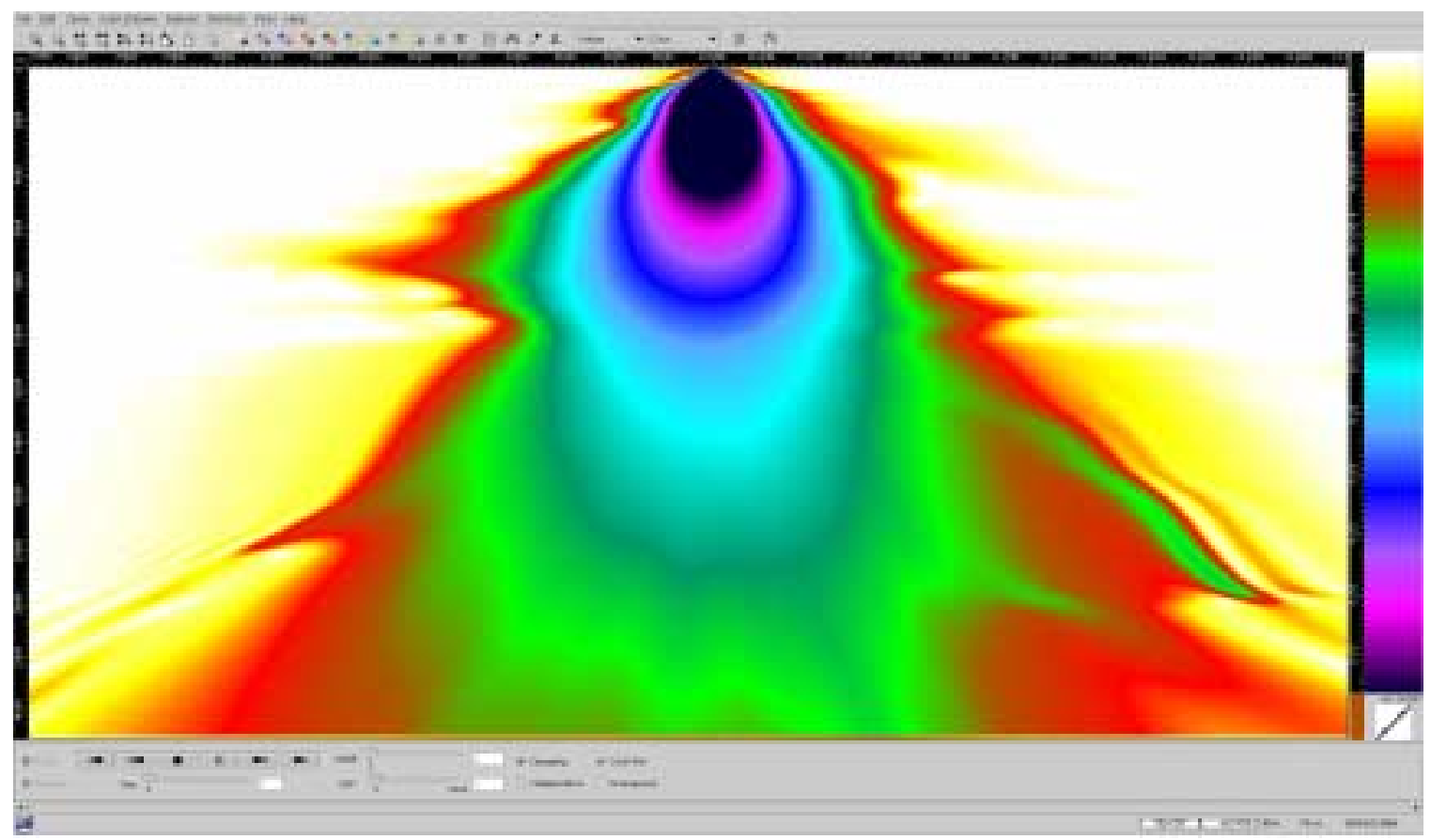

Figure 3.1: Wave-equation illumination at $5 \mathrm{~Hz}$.

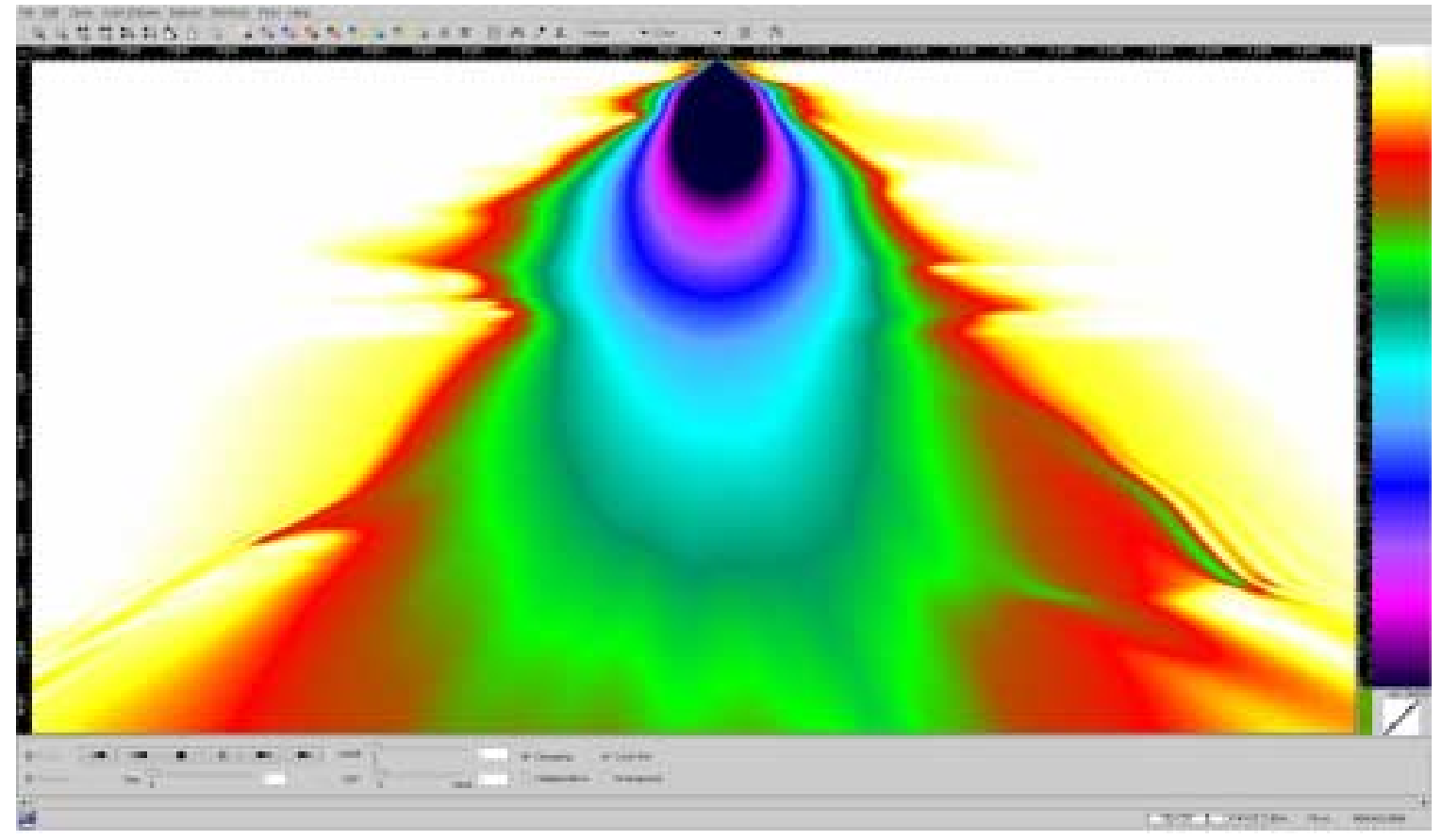

Figure 3.2: Wave-equation illumination at $10 \mathrm{~Hz}$. 


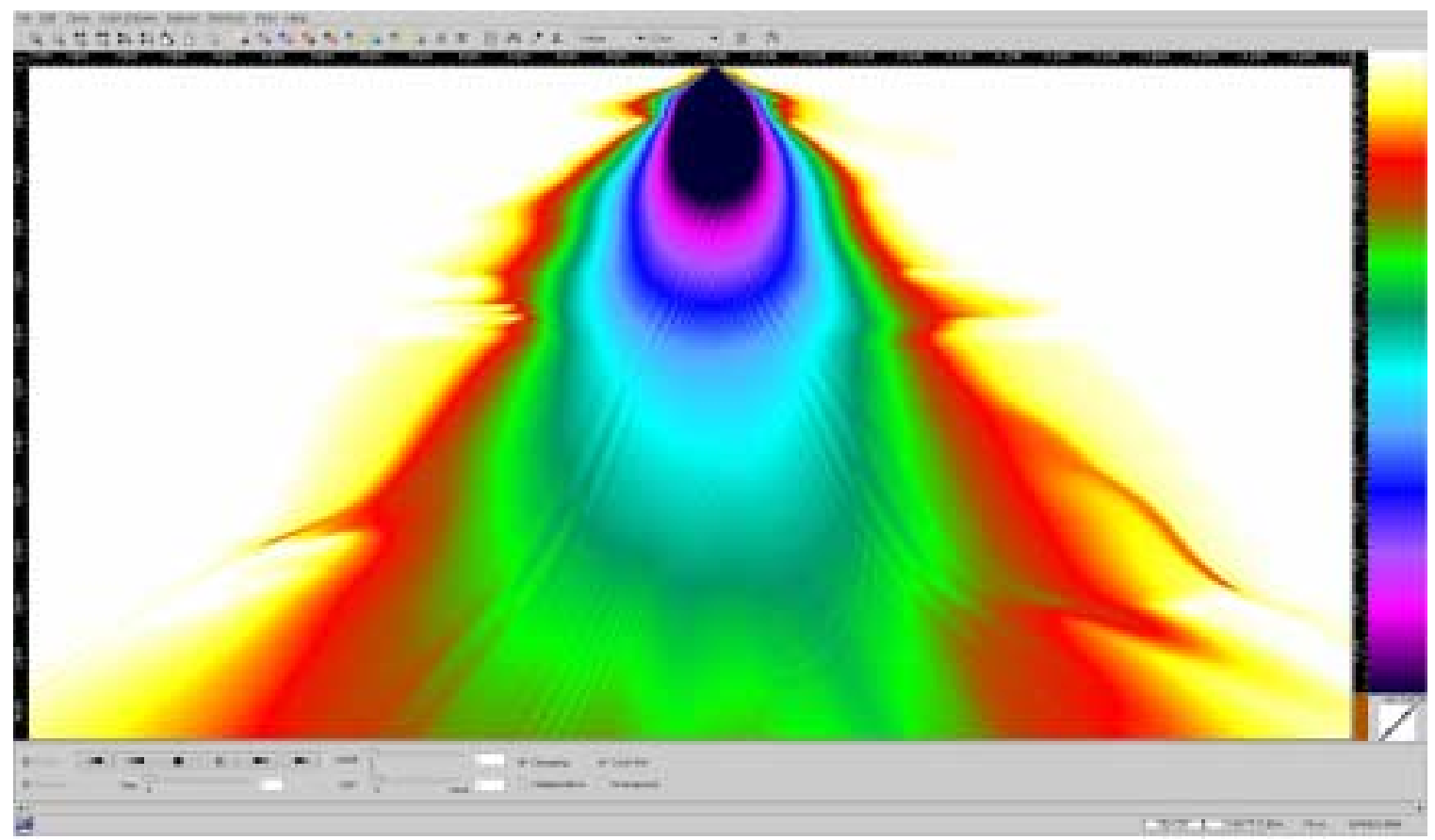

Figure 3.3: Wave-equation illumination at $20 \mathrm{~Hz}$

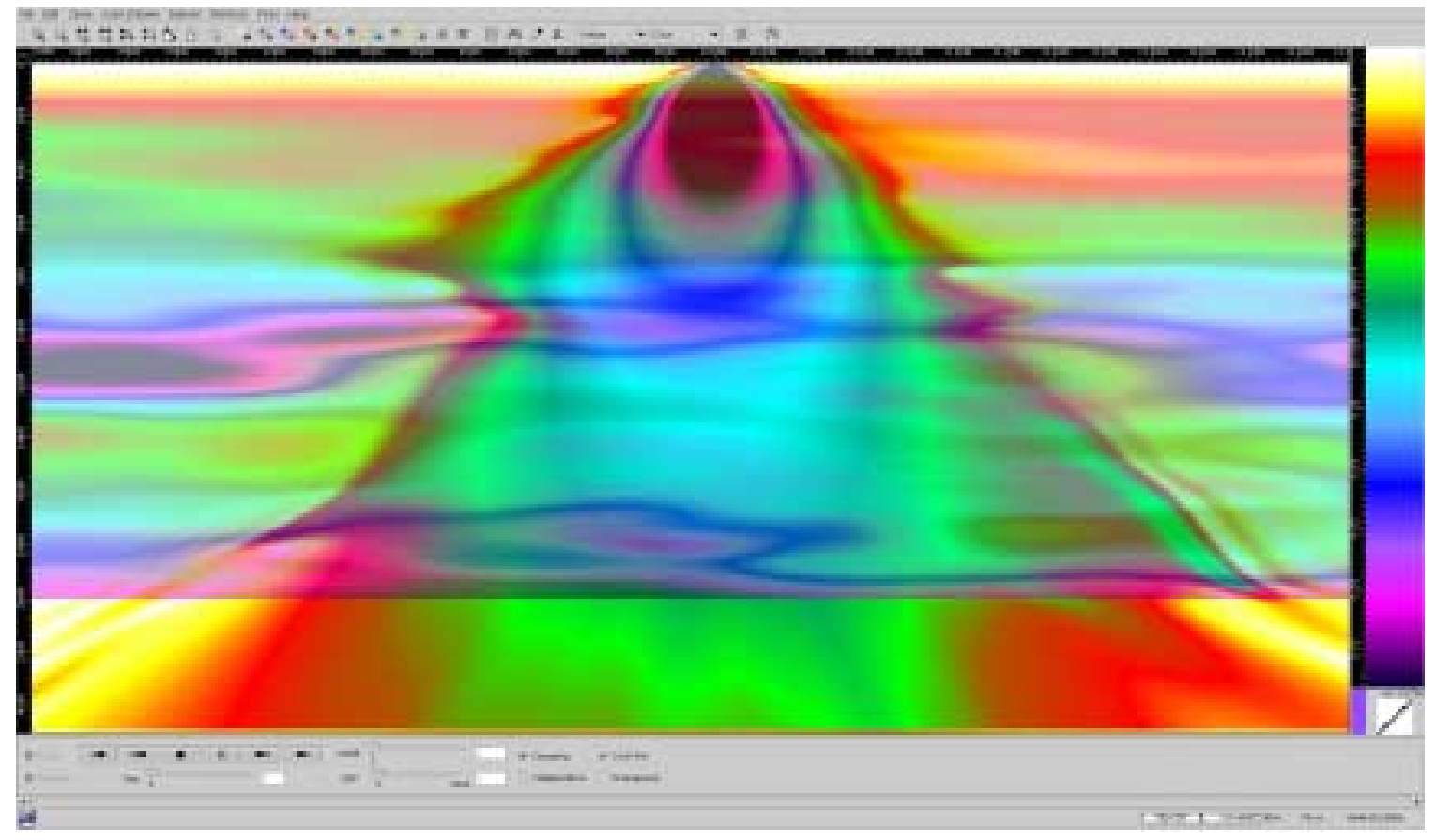

Figure 3.4: Wave-equation illumination at $5 \mathrm{~Hz}$ superimposed on the velocity model. 

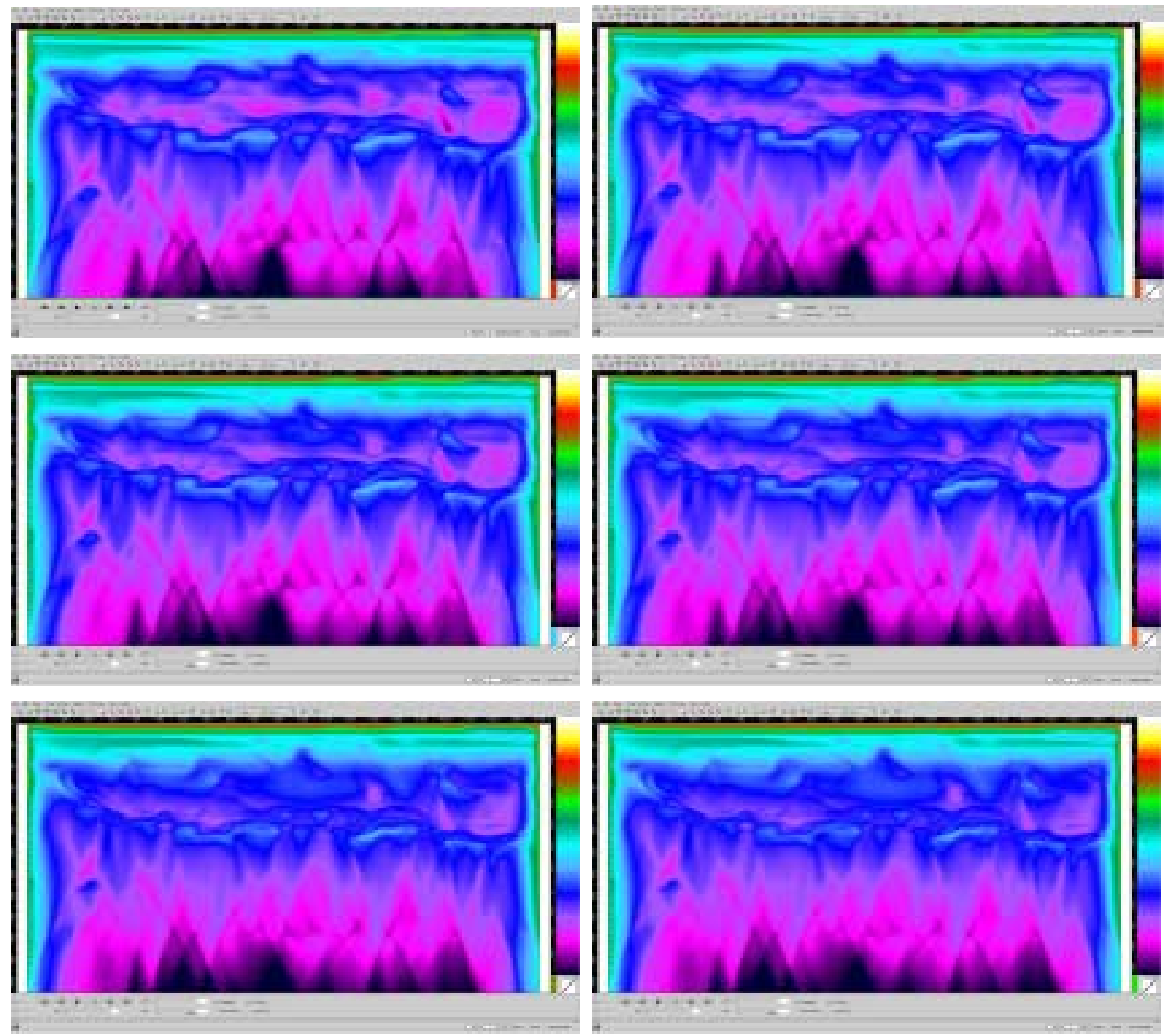

Figure 3.5: Total illumination with $5 \mathrm{~Hz}, 8 \mathrm{~Hz}, 10 \mathrm{~Hz}, 12 \mathrm{~Hz}, 18 \mathrm{~Hz}, 20 \mathrm{~Hz}$.

The Figures 3.5 show the effect of total illumination (all the individual shots summed) using the final velocity model. The amplitude variations are smooth, and after illumination compensation there is no correlation between high amplitude illumination effects and areas of high amplitude in the final imaging results shown in Task 5.

\section{Task 4: Investigate non-elastic attenuation and suggest approaches for recovery}

Non-elastic attenuation of seismic waves leads to the loss of high-frequency energy as the waves propagate through the Earth. As a result, the resolution of seismic images is degraded at large depths. This effect becomes especially troublesome in ultra deep images. 


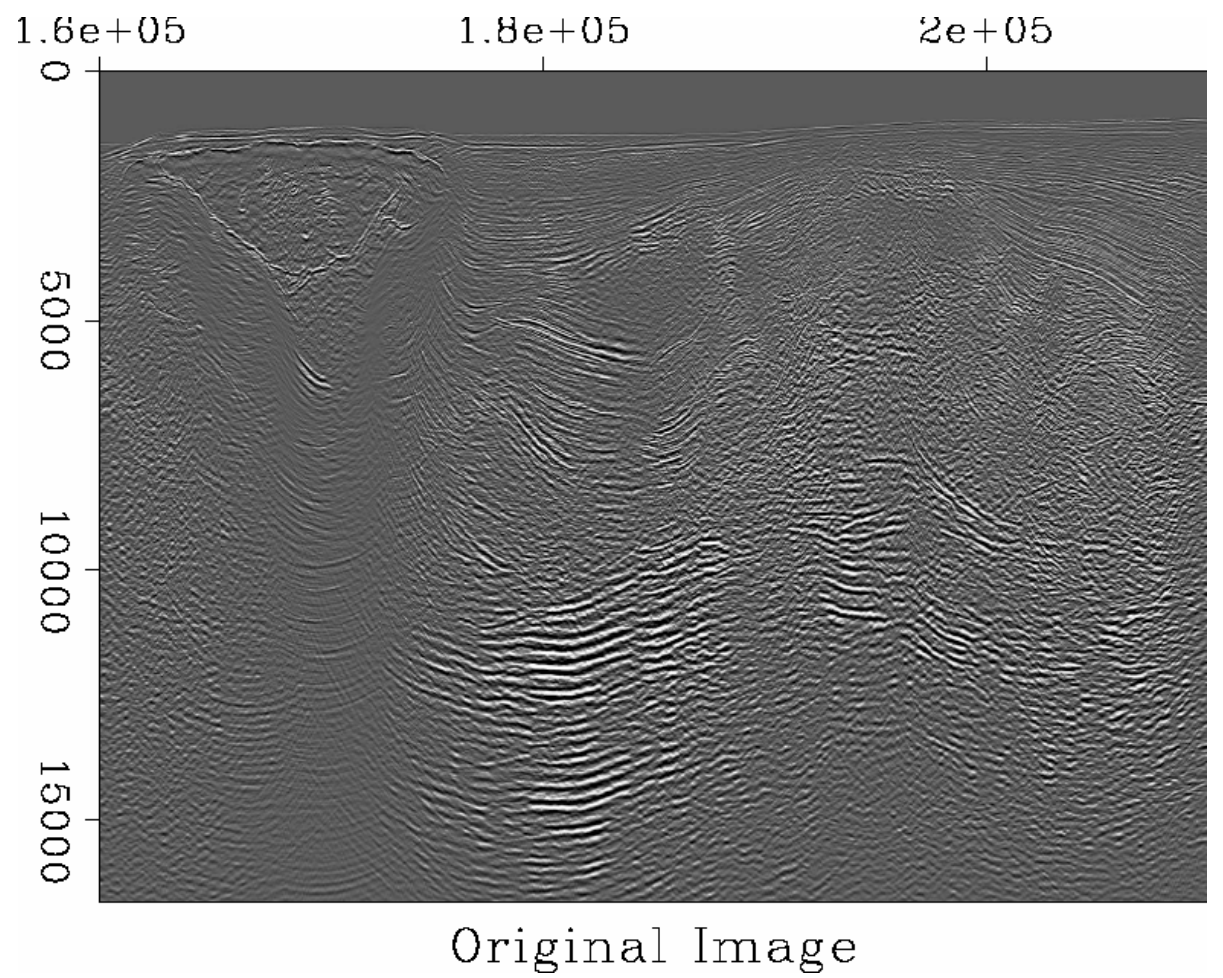

Figure 4.1. Example of a deep image from the Gulf of Mexico. The decrease of seismic frequency with depth, caused by non-elastic attenuation, is clearly visible in the deep parts of the image.

Measuring attenuation is a difficult and open problem by itself. A special conference (Development and Production Forum) was organized by the Society of Exploration Geophysicists in May 2005 to address the problem of estimating seismic attenuation. Traditional local frequency measures such as the instantaneous frequency attribute have a long history (Taner et al., 1979). Such attributes attempt to measure seismic frequency characteristics as being attached instantaneously to each signal point. This measure is notoriously noisy and may lead to unphysical values such as negative frequencies.

Fomel (2005) has introduced the concept of local attributes. Local attributes measure frequency characteristics not instantaneously at each data point but in a local neighborhood around the point. Since frequency, according to the uncertainty principle, is essentially an uncertain characteristic when applied to a local region in the time domain, local attributes are more physically meaningful that instantaneous attributes. They can be used for a robust detection of seismic attenuation. Mathematically, if the analytical seismic trace is represented as

$\hat{f}(z)=f(z)+i h(z)=A(z) e^{i \varphi(z)}$,

where $f(z)$ is the real trace, $h(z)$ is its Hilbert transform, $A(z)$ is the instantaneous amplitude, and

$(z)$ is the instantaneous phase, then the instantaneous frequency is defined as

$\omega(z)=\frac{d \varphi}{d z}=\operatorname{Im}\left(\frac{d \ln \hat{f}}{d z}\right)$

and commonly computed by taking the ratio (Taner et al, 1979) 
$\omega(z)=\operatorname{Im}\left(\frac{d \ln \hat{f}}{d z}\right)=\operatorname{Im}\left(\frac{1}{\hat{f}} \frac{d \hat{f}}{d z}\right)=\frac{f(z) h^{\prime}(z)-f^{\prime}(z) h(z)}{f^{2}(z)+h^{2}(z)}$.

The main idea behind the new local frequency attribute is to recognize the division in equation (3) as a diagonal matrix inversion. In a vector notation, the instantaneous frequency vector $\underline{\omega}$ is

$\underline{\omega}=\underline{D}^{-1} \underline{n}$,

where $\underline{n}$ represents the numerator in equation (3) and $\underline{D}$ is the diagonal matrix corresponding to the denominator in equation (3). The local frequency attribute (Fomel, 2005) appears from the regularized version of equation (4), as follows

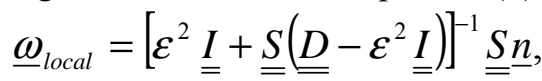

where $\underline{\underline{I}}$ is the identity operator, $\underline{\underline{S}}$ is the smoothing operator that introduces local regularization, and is the regularization parameter.

In this project, we have implemented the local frequency estimation algorithm and tested it on real data examples. Figure 15 shows an estimate of the local frequency from the image in Figure 4.1. For a physically meaningful result, we display the local wavelength defined as

$\lambda_{\text {local }}=\frac{2 \pi}{\omega_{\text {local }}}$.

Local wavelength is a measure of seismic resolution. It indicates the scale of geological features resolvable in a seismic image. We can see that the resolution decreases dramatically in the deep part of the image as the local wavelength increases from about 30 meters near the surface to 200 meters in the deep part of the section.

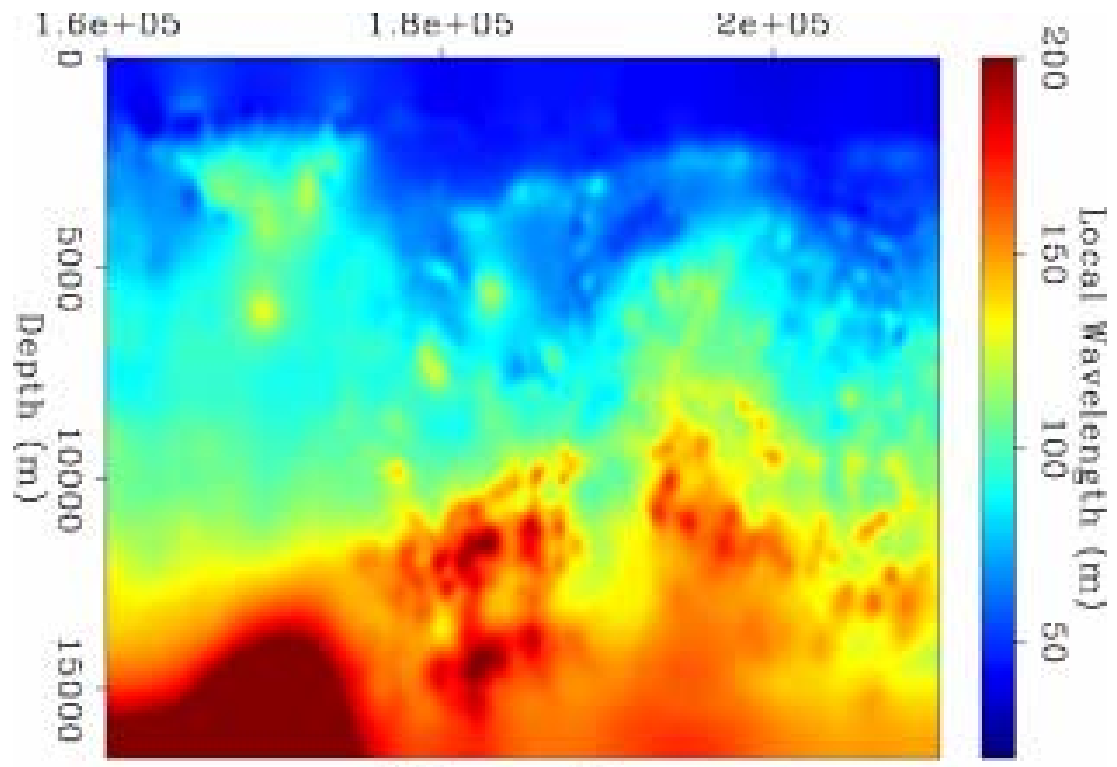

\section{Attenuation}

Figure 4.2. Estimated local dominant wavelength using the new robust local frequency attribute. At deep targets, the wavelength increases to more than $100 \mathrm{~m}$ because of attenuation, thus decreasing the imaging resolution. 
Once a reliable measure of seismic attenuation is obtained, we can devise a numerical scheme for restoring the attenuated parts of the image. In the literature on astronomical imaging, the restoration process is known as deblurring. Deblurring treats attenuation as local smoothing (blurring) and attempts to invert the smoothing process. In that sense, it is analogous to nonstationary seismic deconvolution.

A comparison between the original and deblurred image for the deep portion of the data is shown in Figure 4.3. One can observe an evident increase in seismic frequency of the signal. Increasing the local frequency does not necessarily helps seismic interpretation but it is crucially important for resolving small features in deep targets. Figure 4.4 shows a measure of the local wavelength after deblurring. As expected, the deblurring process equalizes the frequency content between shallow (non-attenuated) and deep (attenuated) parts of the image. Deblurring attempts to restore the attenuated parts of the image, as shown in Figure 4.5, by comparing average spectra of the two images. Of course, such a restoration is highly non-unique and should be used with caution. It is meaningful only when supported by independent information such as well log analysis. A combination of well log data, seismic resolution measurements, and deblurring will provide a new unique tool for high-resolution imaging of ultra deep exploration targets. Future work at $3 \mathrm{DGeo}$ will create commercial tools for including well log and geological information in the software products for non-elastic attenuation.
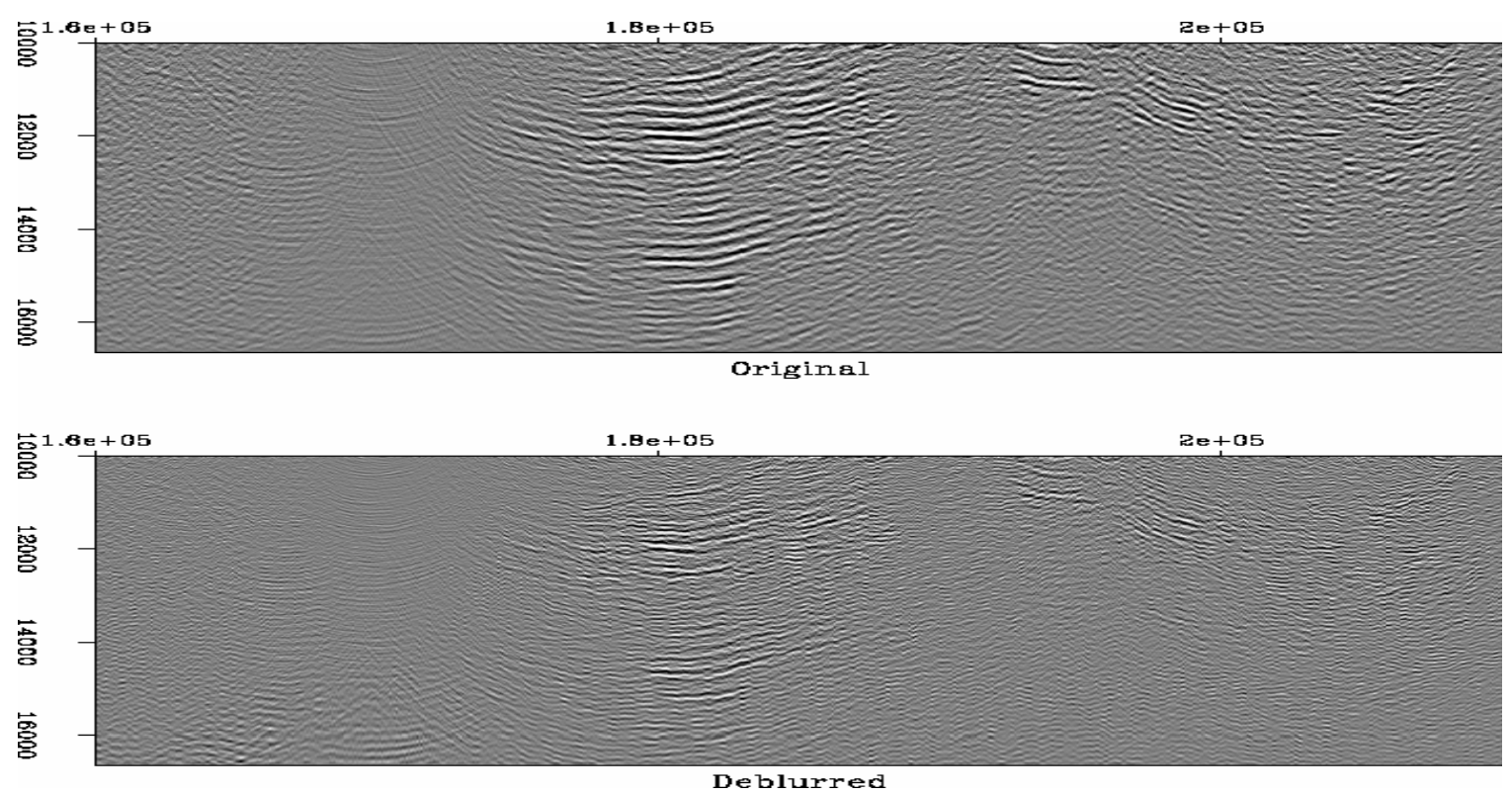

Figure 4.3. Comparison of the original and deblurred image for the deep part of the image, the bottom image has higher frequency. 


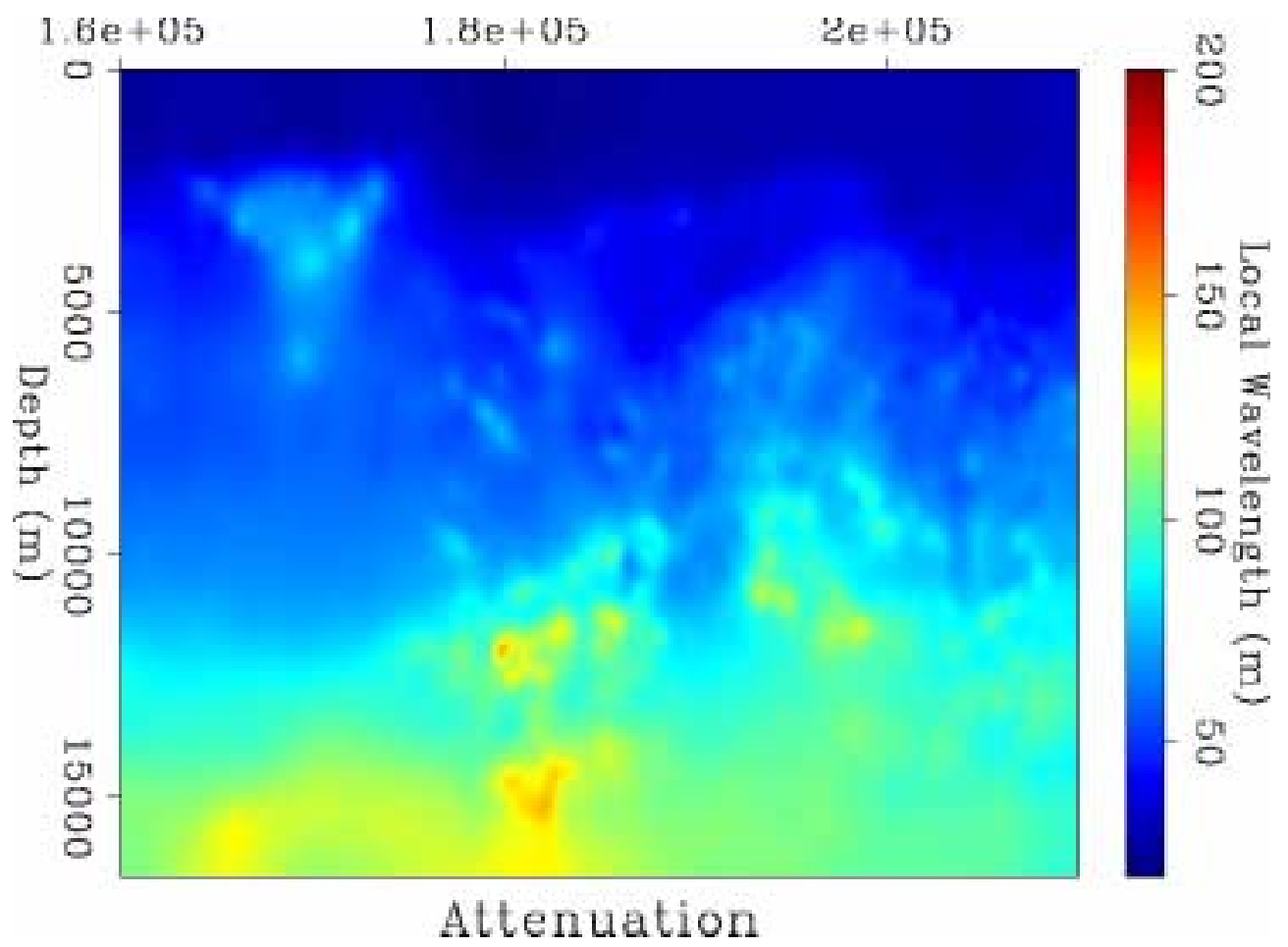

Figure 4.4. Local seismic wavelength measure after deblurring,

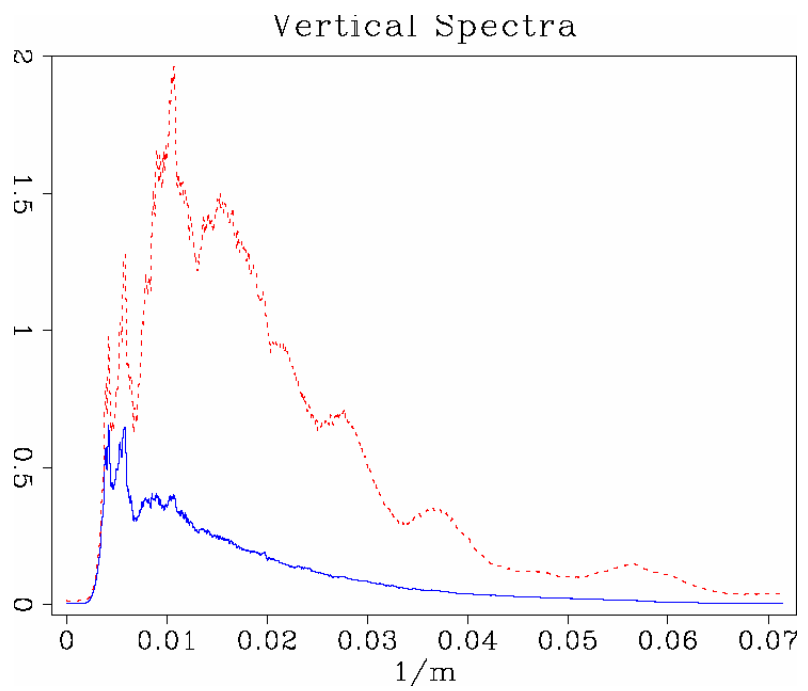

Figure 4.5. Average vertical spectrum before (blue solid line) and after (red dashed line) deblurring, Deblurring restores attenuated parts of the signal. The restoration is non-unique and further research is needed to stabilize and constrain the operation.

\section{Task 5: Demonstrate imaging and accuracy on ultra-deep data}

The vast majority of seismic data in the Gulf of Mexico and onshore Texas has been recorded with relatively short offsets (seldom exceeding 16,000') and with insufficient record lengths (6 to 8 seconds), for imaging shallower structures onshore and offshore. To address the need for acquisition of seismic data appropriate for ultra-deep imaging Petroleum Geo-Services (PGS) has 
acquired a proof of concept (POC) line, using very large offsets, 1,000 channels live (splitspread) onshore, and 500 channels live offshore (simulated split-spread) with 250 fold, a record length of 20 seconds, and maximum offsets of 41,200 feet (12,500 meters) in the upper Texas coast using dynamite and Airgun sources. The location of the POC line is shown in Figure 5.1. This is a very appropriate dataset for testing ultra-deep technology, since it has a very long record length and large offsets. The length of the record allows us to image very deep structures and steep dips, while the large offsets offer good data redundancy and the ability to better discriminate the velocity of the deeper structures. This is an ideal dataset for this project and comes with several challenges. The shot gathers show packets of coherent energy at large times, indicating that there is structural information in the deep data, but at the same time the stacked data shows well defined structures to 7-8 seconds, after which the image becomes incoherent. One of the challenges of this project is to bring out this deeper information and image the geological structures deeper than 7-8 seconds.

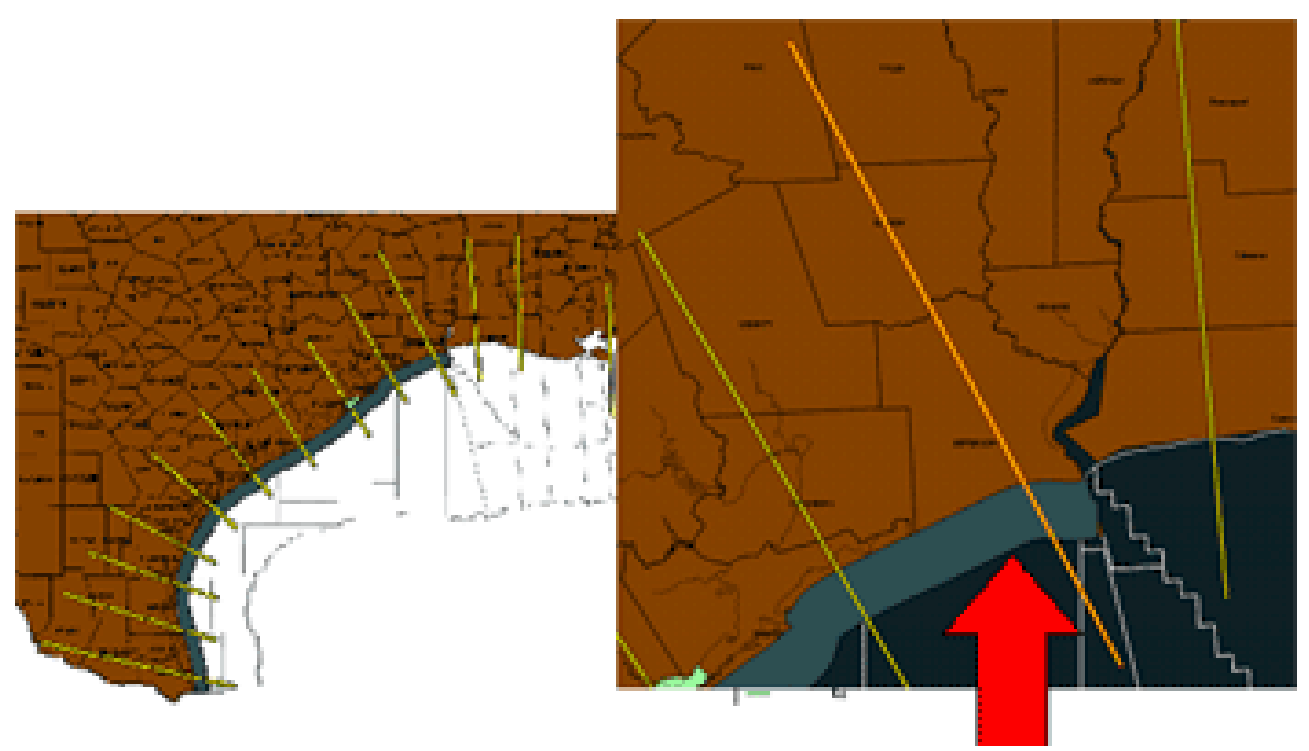

Figure 5.1. :Location the $\mathrm{POC} 2$ line.

The gather data shown in Figure 5.2, was deemed to be of good quality with some high frequency noise scattered throughout the line. The high frequency noise was easily filtered out with a simple bandpass filter. The gather data was reviewed with and without normal move-out applied to determine the accuracy of the stacking velocities which were provided by PGS.

A sparse velocity analysis was run to check if there was residual velocity which could be corrected easily and to see if multiples contamination was present and if there was enough delta-t for removal of multiples without removing the primaries. The velocities needed very minimal adjustment, some multiples were present in the mid to far offset ranges, between 5 to 9 seconds, but did not contaminate the entire line. The line was stacked with the stacking velocities provided. The stack response was of good quality with reasonable data coherency appearing in the deeper section. Three offset stacks were generated to view the contribution of the offsets; near offset stack with ranges of 0 to 4160 meter offsets; mid offset stack with ranges of 4160 to 8320 meter offsets, and far offset stack with ranges of 8320-12500 meter offsets.

The summation of adjacent CDPs to try and produce better signal to noise ratios were done on a 2-1 sum, 4-1 sum and 8-1 sum. This process combines traces together into output ensembles, while preserving the original traces. The traces are then summed together based on a series of bins determined by offset ranges. All traces in a bin are stacked to form a single trace within the ensemble. The traces were normal move-out corrected before being summed. The output stacks 
created improved the signal to noise ratio over the normal stack. The 2-1 sum was a slight improvement over the normal stack, the 4-1 sum was good improvement over the normal stack and the 2-1 sum stack. The 4-1 sum stack improved the overall signal but in particular the deeper data while preserving the diffraction patterns. The 8-1 sum also improved the deeper data but did not preserve the diffractions. A post stack Kirchhoff migration was run on the normal unsummed stack data. The post stack migration did not reveal anything significant that could not be perceived in the stack.

The stacking velocity was used for an initial run of pre-stack time migration (PSTM). The PSTM velocity was updated for an improved pre-stack time migration run. The updated PSTM velocity was converted to interval velocity and served as a starting model for a wave-equation depth migration run. The depth velocity model was updated through successive iterations of migration and velocity update using normal ray and tomographic updates.

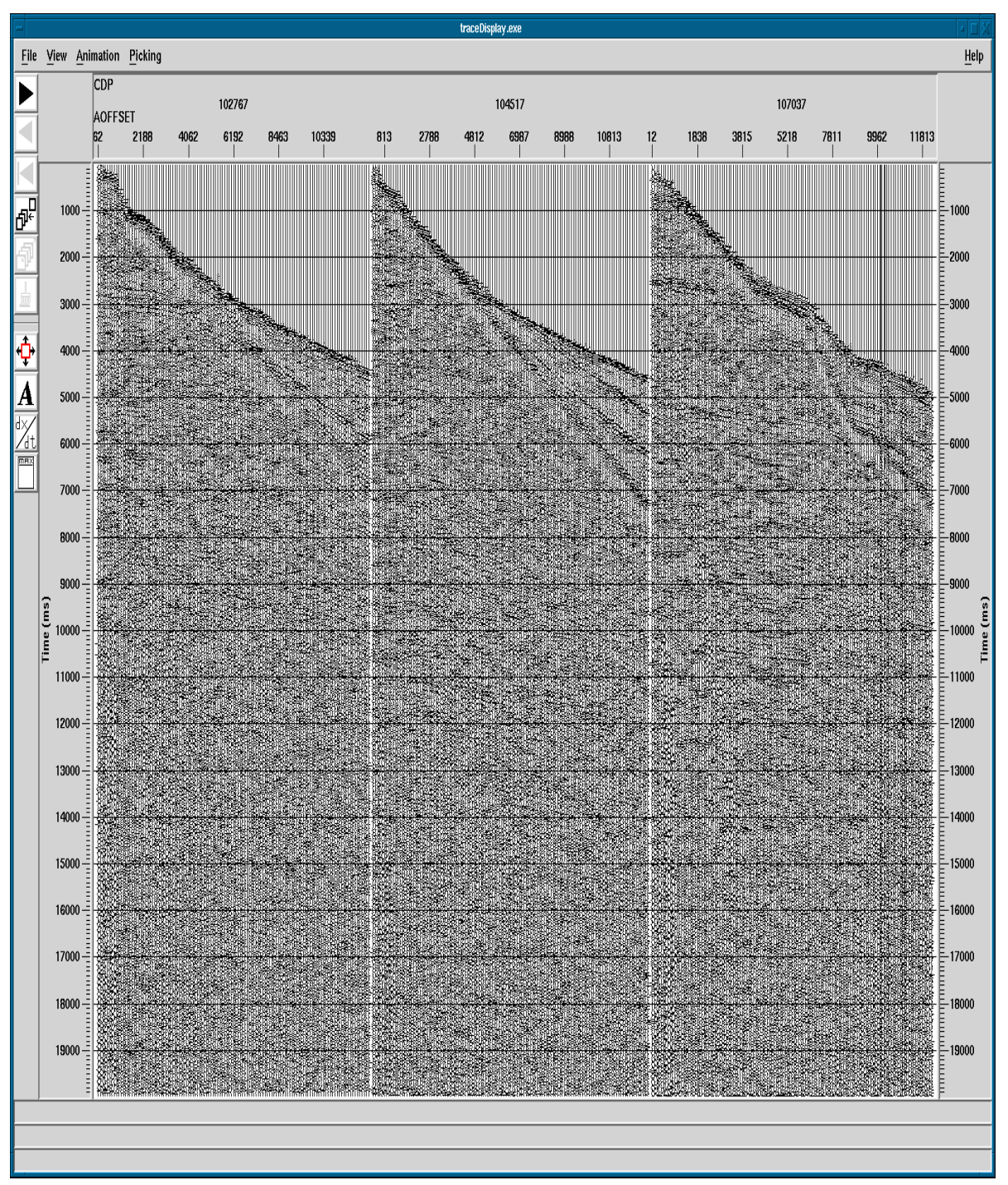

Figure 5.2: Raw shot records. Notice the large offset and 20 seconds recording time. Data courtesy of PGS. 
Figure 5.3 shows a post-stack time migration of a part of the POC2 line, and Figure 5.4 shows the DMO velocity model. Figure 5.5 shows the pre-stack time migration of a part of the POC2 line.

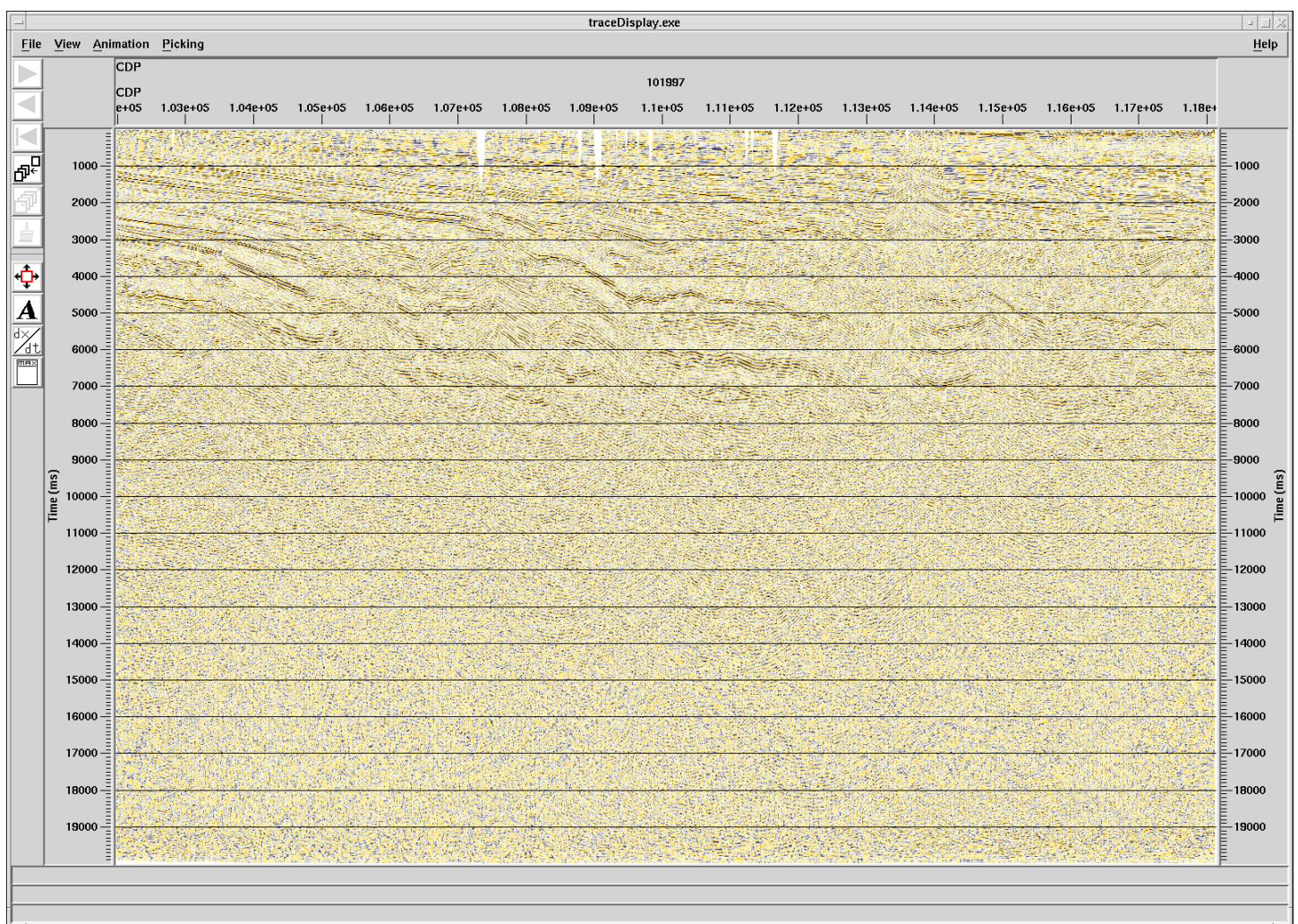

Figure 5.3. Post-stack time migration. Data courtesy of PGS. 


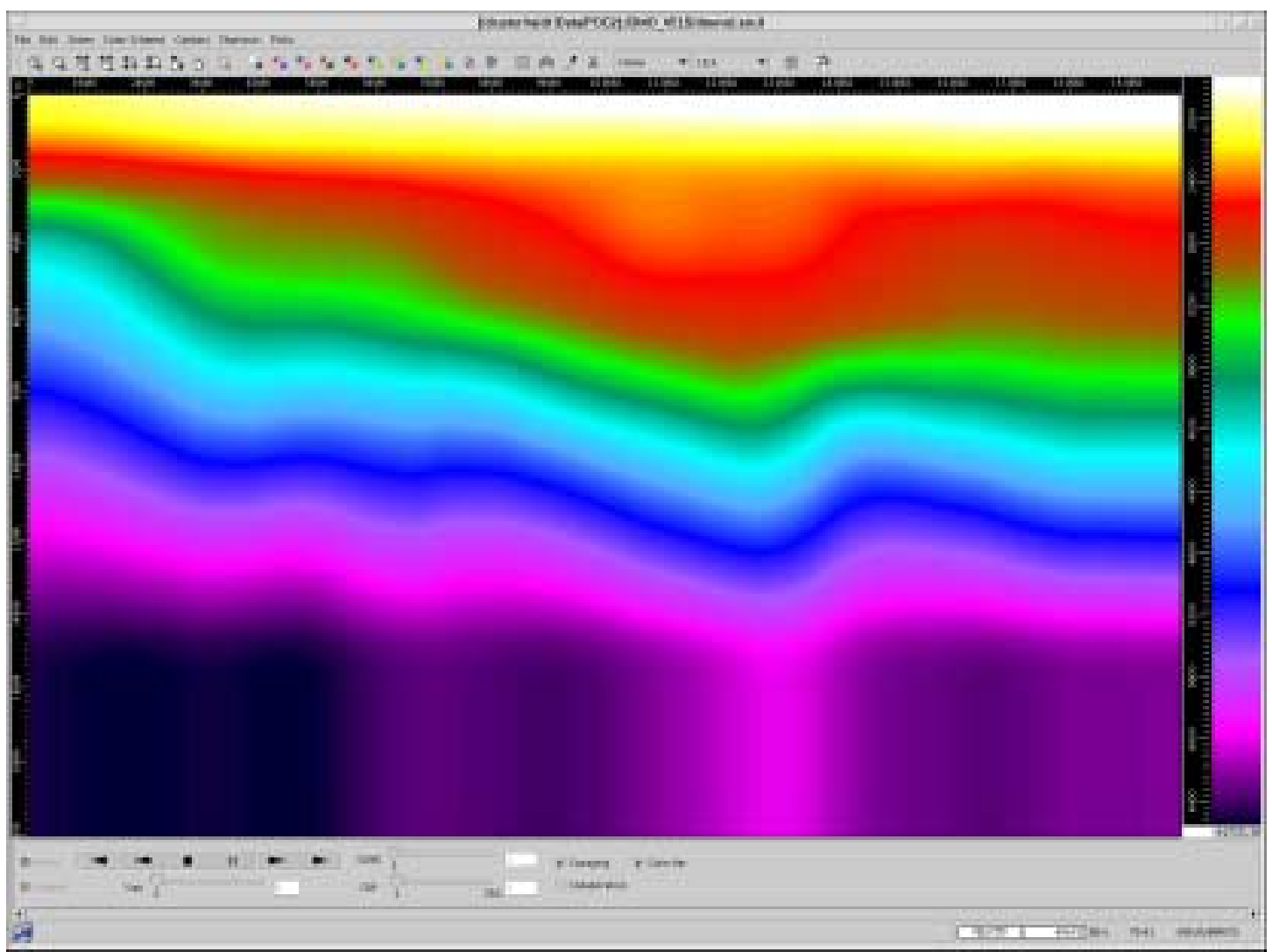

Figure 5.4. Initial DMO velocity model.

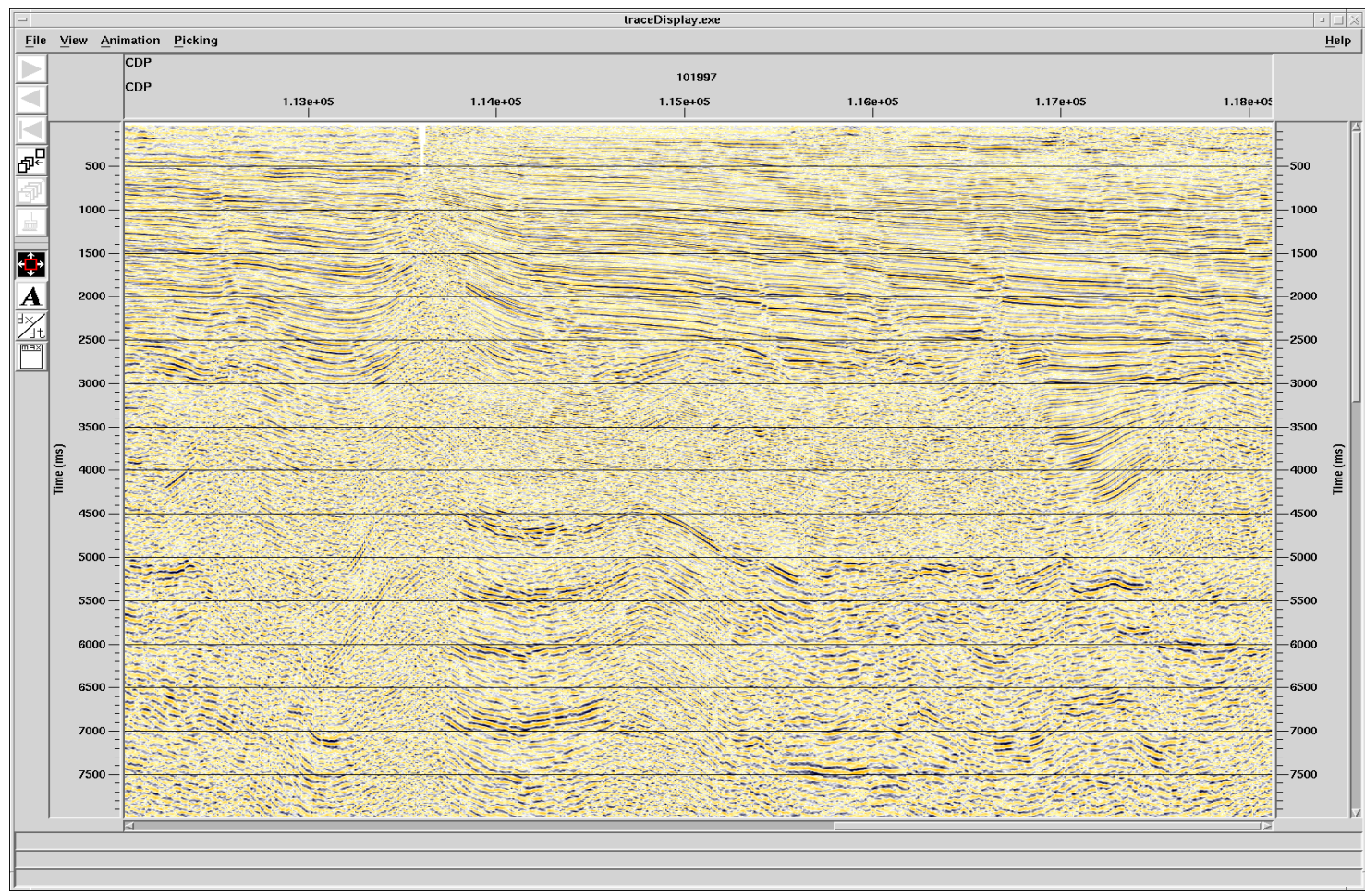

Figure 5.5. Filtered pre-stack time migration, right side of the line. Data courtesy of PGS. 
We ran several data regularization tests to optimize the azimuth moveout (AMO) parameters. Figures 5.5 and 5.6 show the effects of AMO on the migrated gathers and the subsequent residual velocity analysis. After AMO the data is preconditioned to improve the quality of the migration velocity analysis (MVA). The migrated image is virtually unaffected by the data preconditioning, the differences between the migrated images of the datasets before and after AMO are very small. However, for velocity analysis, the AMO pre-conditioned gathers generated much tighter semblance panels as shown in Figure 5.8. The semblance panel in Figure 5.8C has a much tighter fairway allowing for a higher resolution picking of the residual velocity values.

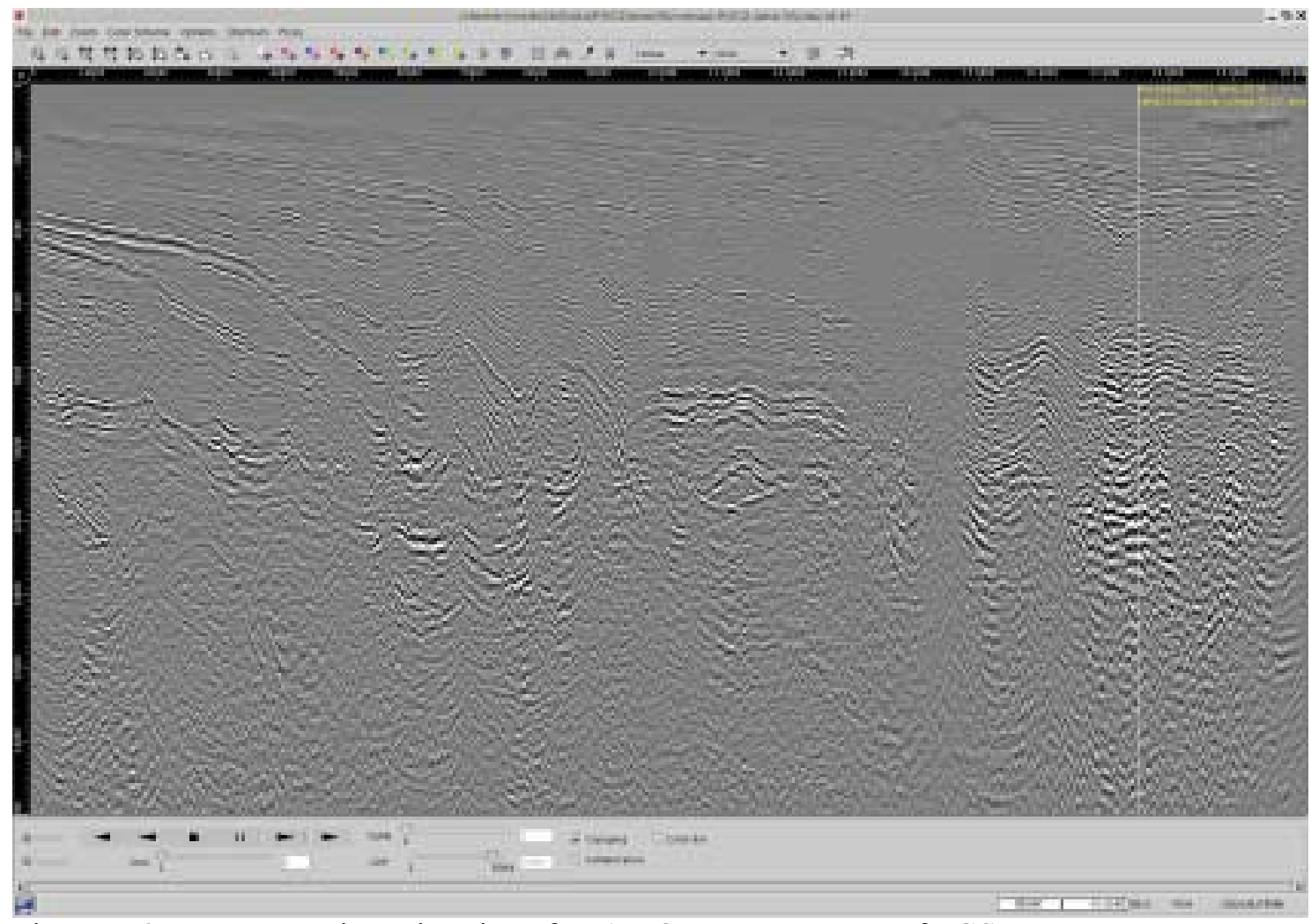

Figure 5.6. Wave-equation migration after AMO. Data courtesy of PGS. 

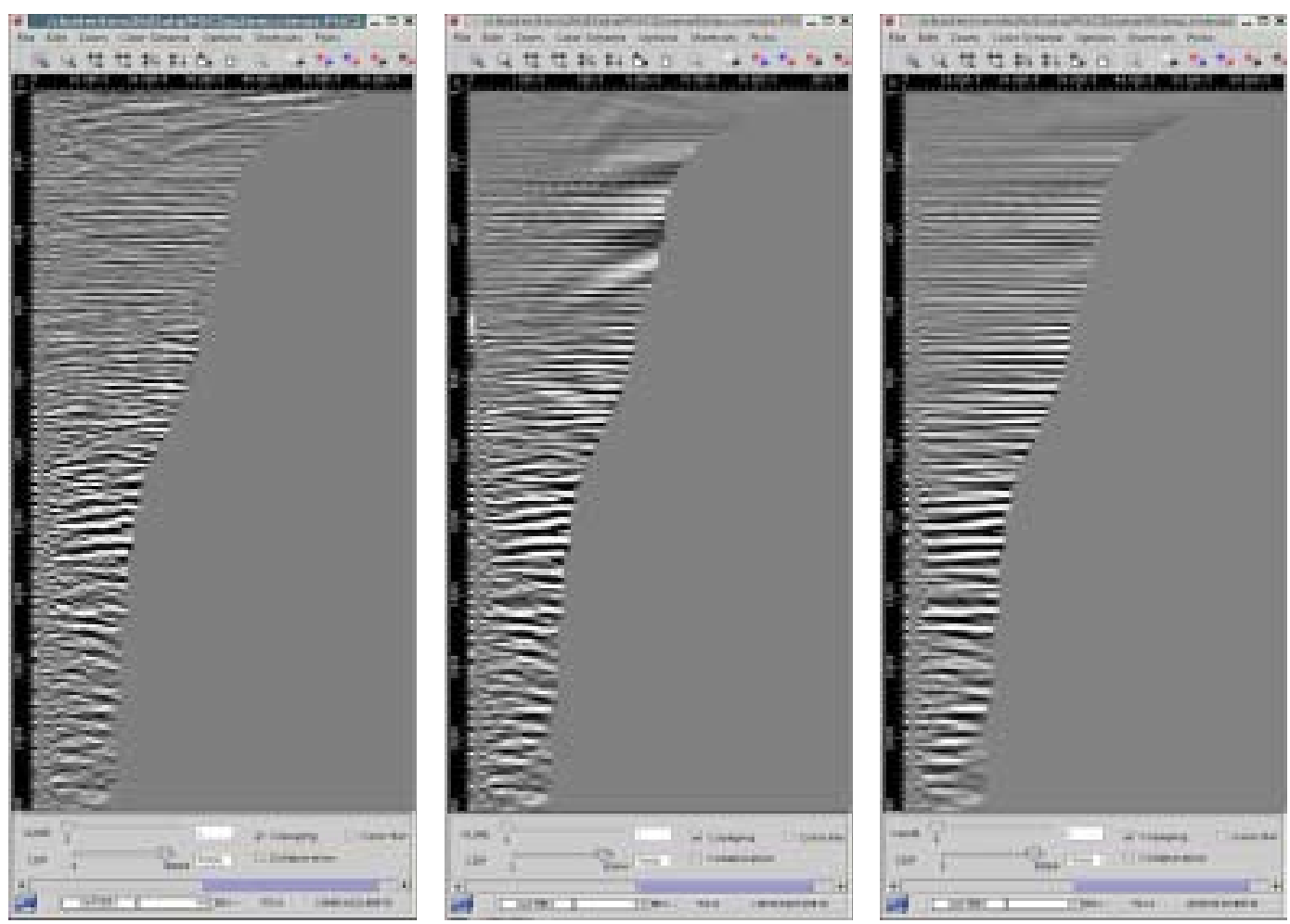

Figure 5.7: Migrated wave-equation angle gathers: A. Before AMO. B. Amo test 1. C. Amo test 2.
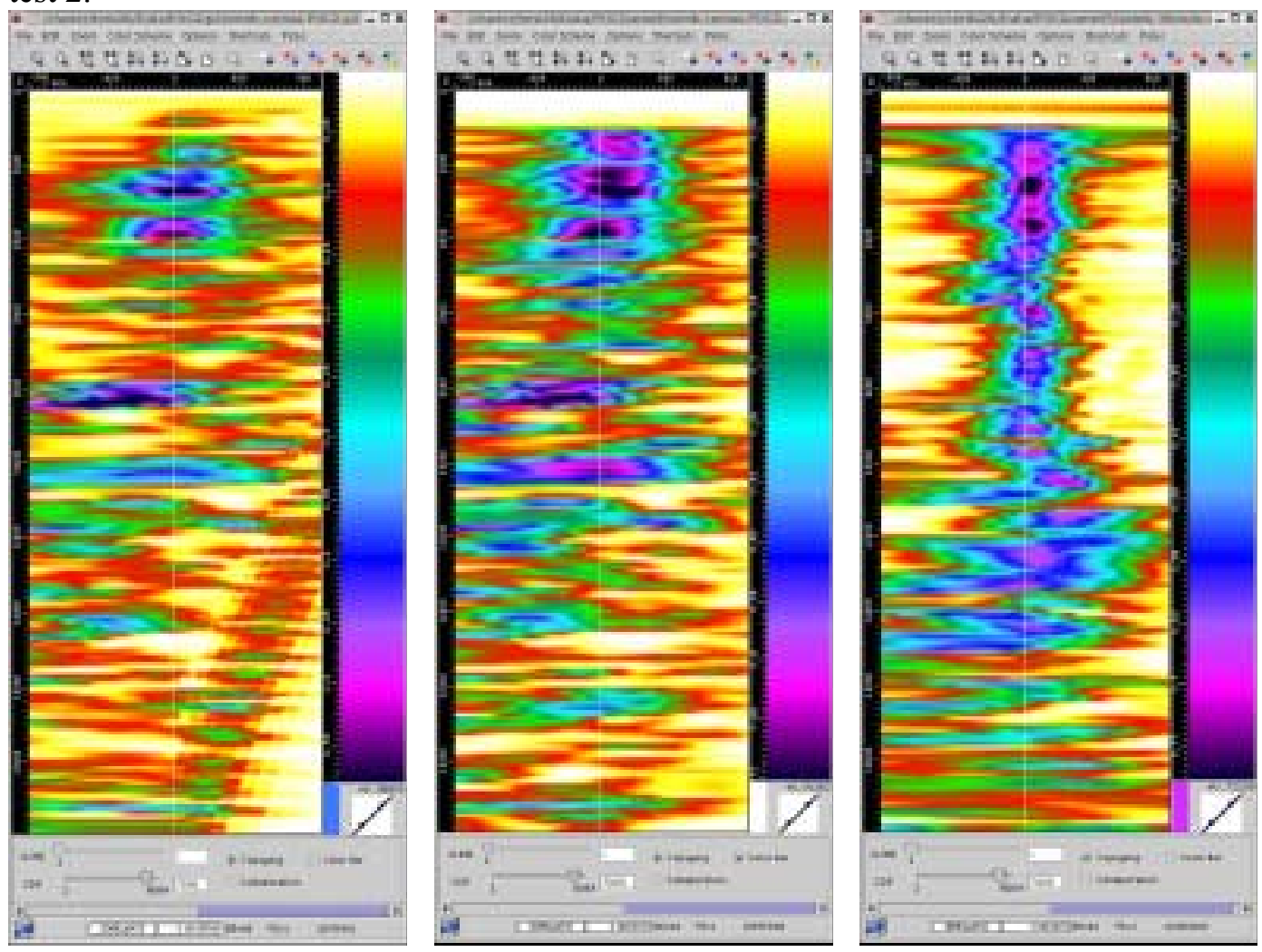

Figure 5.8: Residual Semblance. A. Before AMO. B. After AMO test 1. C. After AMO test 2. After AMO test 2 has the semblance becomes better defined. 


\section{Tomography}

An important step in the wave-equation prestack depth migration is the velocity model building process. The most accurate way of reconstructing velocity models is prestack migration velocity analysis (MVA) based on angle domain common image gathers (ACIG). The ACIGs contain redundant structural information that is used to correct the initial velocity model. Furthermore, velocity model building is most accurate if updates are based on prestack gathers generated from the same imaging algorithm that is used for the final imaging step. It is therefore imperative that wave-equation migration imaging is intimately related to wave-equation MVA. Given a set of seismic data and an initial velocity model, angle domain common image gathers are generated for the volume of interest. From these ACIGs, we extract residual velocity information by scanning over angle or ray parameter. The scanning formula takes into account the relationships among migration depth, migration velocity, residual velocity, and ray parameter/angle. Once the semblance panels are generated, the residuals are backprojected into the overburden medium and used to update the initial velocity model by tomography.

We conducted several tests on improving the velocity model using several iterations of tomography. The purpose of the tomography tests is to update the velocity model and make it possible to get a more accurate image. The tomography and automatic event detection and picking is based on scanning the seismic data volume with prediction-error filters and automatically selecting back projection points based on dip coherency and semblance strength. The dip estimate is refined using the methodology described in Fomel (2000), and back projection points are automatically selected based on dip coherency and semblance strength. The major procedures involve picking reflection points, tracing rays, inverting for the residual moveout in the depth migrated angle gathers, and updating the velocity model. The reflection point picking procedure generated good results, which after smoothing produced the image displayed in Figure 5.9.

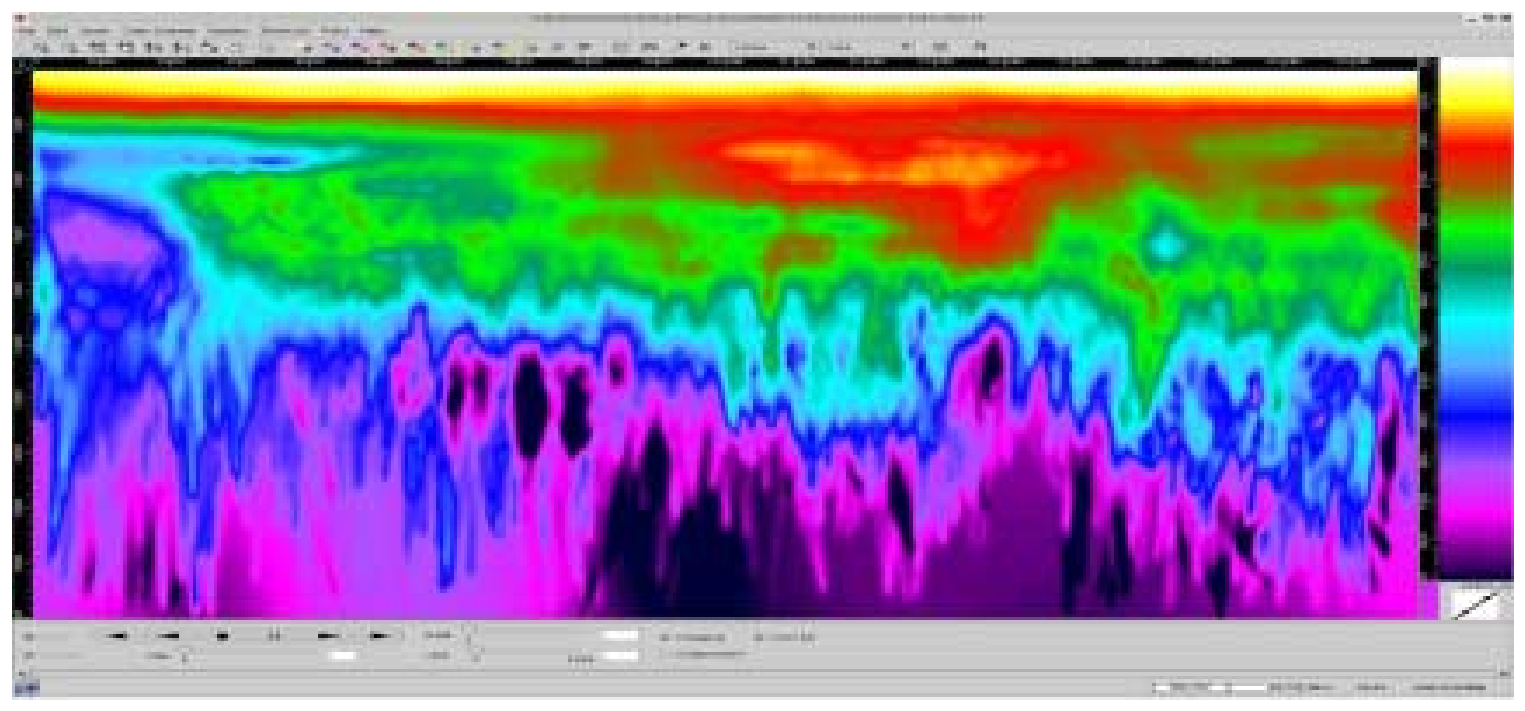




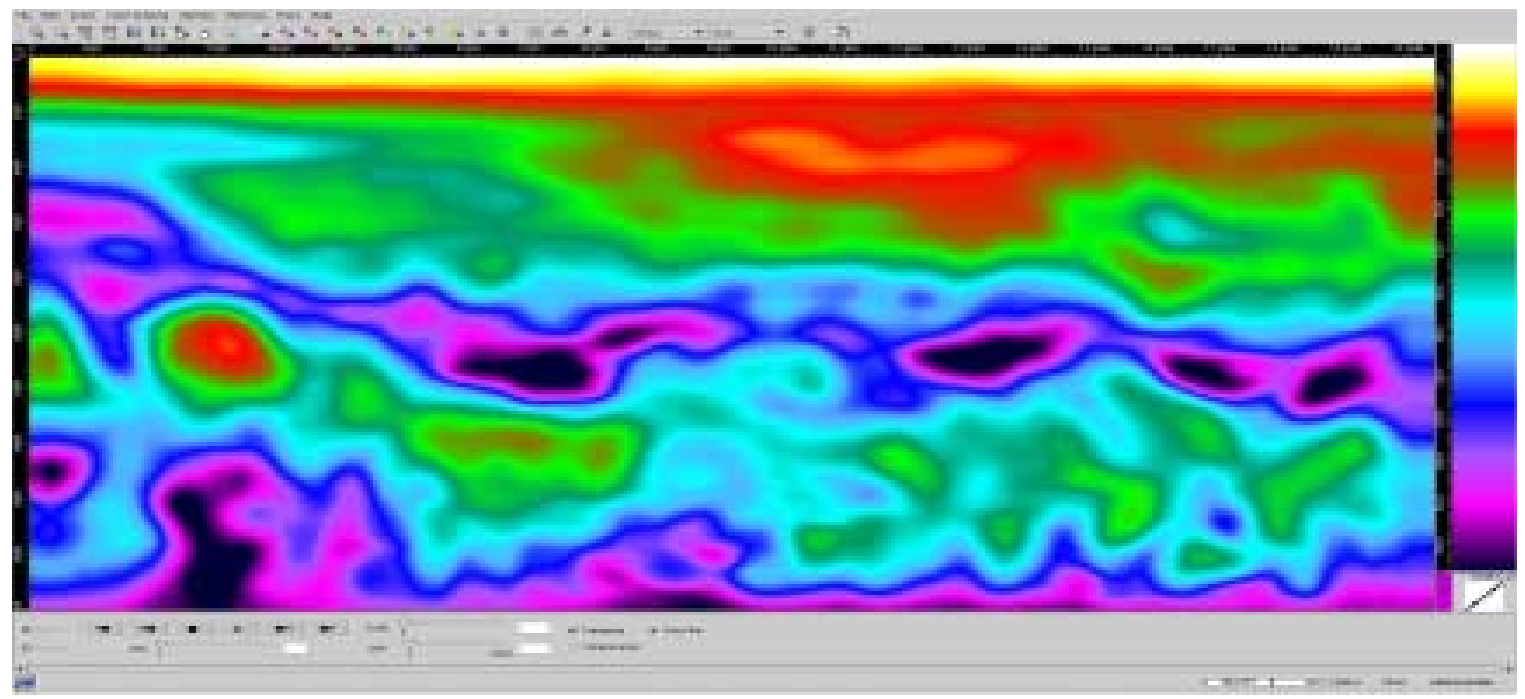

Figure 5.9. Tomography iterations of POC 2 data. Top, early iteration. Bottom, final model.

\section{Improve the focusing of ultra-deep events}

We have performed several tests on improving the coherency of deep and ultra deep events. First set of tests established optimum functions for boosting lower frequencies in the downward continuation part of the migration, and the second set of tests analyzed post-migration processing for flattening non-hyperbolic (parabolic for angle-gathers) move-out.

Figure 5.10 shows that some of the horizons in the deeper part of the angle gathers have a moveout that can not be parameterized by a single residual velocity value. Therefore, regardless of how many velocity iterations we do, we will not be able to correct this via standard residual semblance based velocity analysis. Therefore we can either attempt post-migration flattening of the gathers, or parameterize the non-hyperbolic moveout with different values for each angle (at constant depth), and use this redundant parameterization with tomography. 

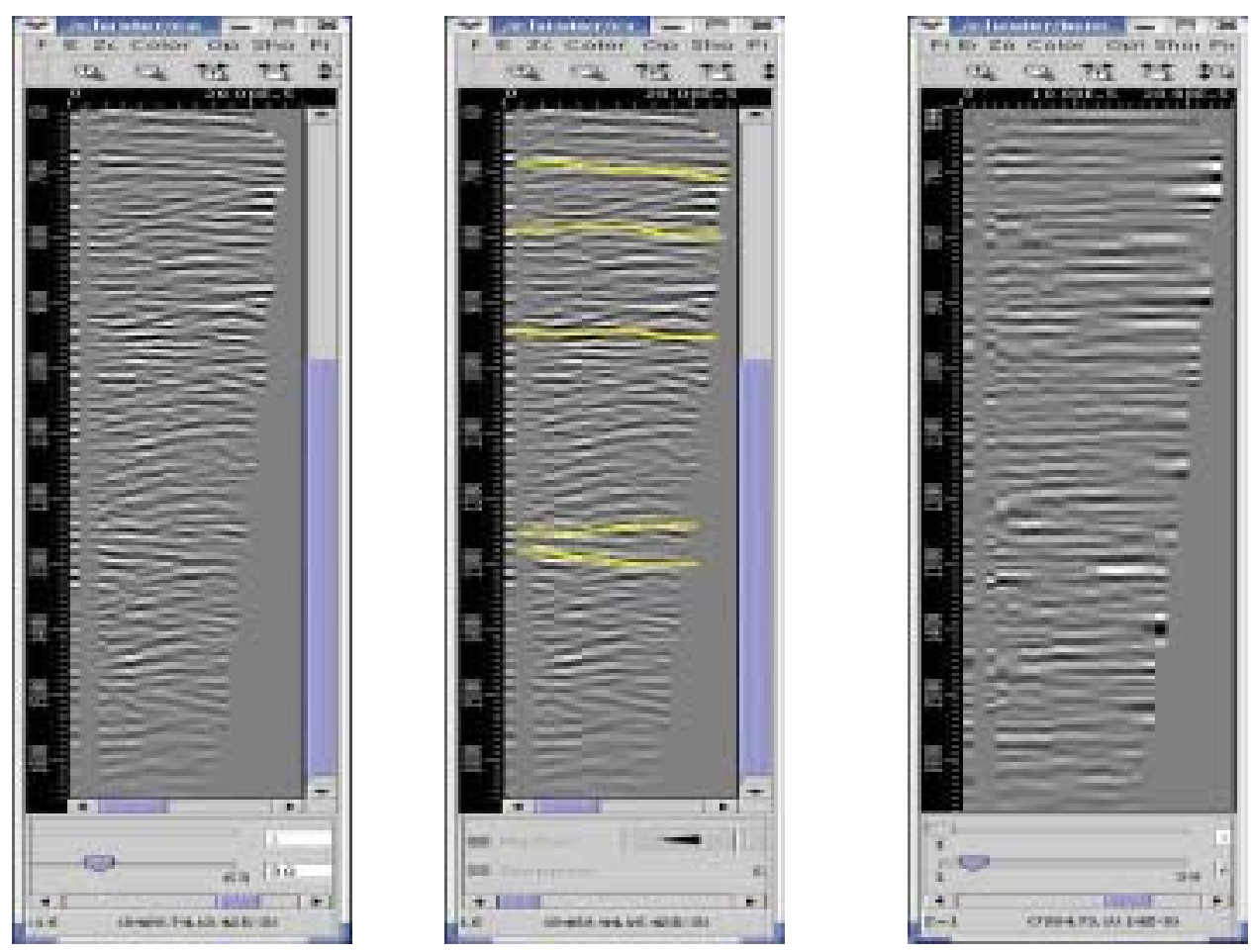

Figure 5.10: Left and Middle: angle gathers showing residual non hyperbolic moveout. Such events are hard to correct through typical velocity analysis based on residual hyperbolic curvature. Right: Angle gather after flattening.

Figures 5.11 and 5.12 show the improvements of the wave-equation depth migration using ultradeep event boosting technology compared to the existing standard Kirchhoff technology. Some of the techniques used to boost the deeper events can be also applied to the standard Kirchhoff, though some of the applications in frequency domain may be limited to wave-equation methods operating in frequency domain. The figures show a small area of the PGS proof of concept line between 5000 meters and 18,000 meters. The deeper structures show better continuity in the wave-equation case, better resolution and allow the interpreter to define and contour structures at depth previously hard to image with standard technology. 


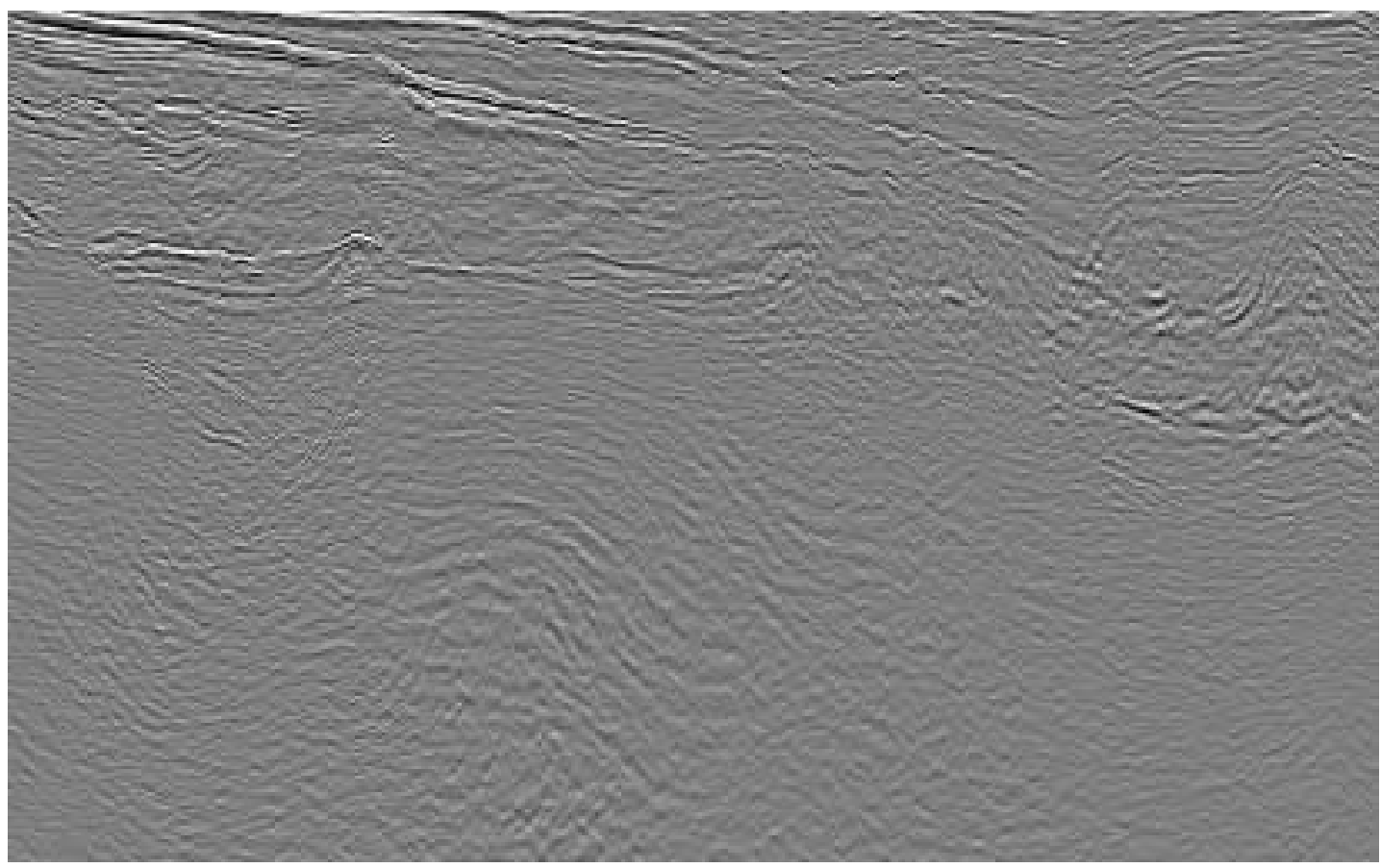

Figure 5.11: Standard processing sequence using Kirchhoff depth migration for final imaging, between approximately $5000 \mathrm{~m}$ and 18,000 $\mathrm{m}$ depth. Data courtesy of PGS.

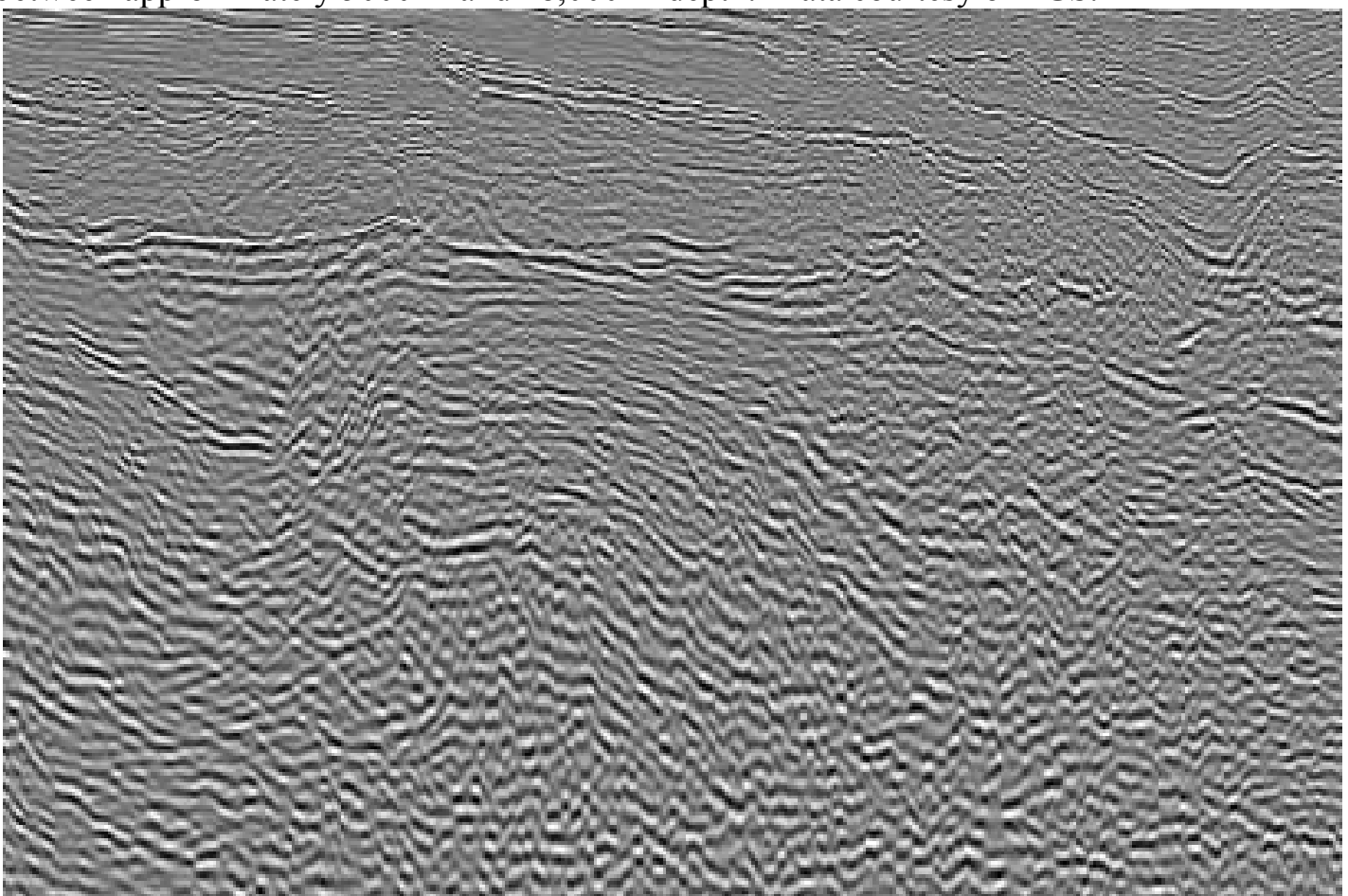

Figure 5.12: Wave-equation depth migration using ultra-deep events boosting technology between approximately $5000 \mathrm{~m}$ and 18,000 $\mathrm{m}$ depth. Data courtesy of PGS. 


\section{Task 6: Investigate effect of aperture on ultra-deep data.}

A series of tests were performed using a variable aperture in Kirchhoff migration to observe the differences in the imaging of the steep and deep events. Figure 6.1 shows the migration result using a 40 degrees migration aperture, while Figure 6.2 shows the migration result using an 80 degrees migration aperture. Apart from some shallow faults there are no major differences between the 40 degrees and the 80 degrees aperture, so the conclusion is that given the appropriate lateral aperture the angle does not make a big difference for the deeper events, since the dips are not very steep in that region. Similar test were performed by using several versions of the dataset and restricting the offset. The data was limited to offsets up to $4000 \mathrm{~m}, 6000 \mathrm{~m}$, and $10,000 \mathrm{~m}$. Each dataset was then migrated using the wave-equation prestack depth migration algorithm. Somewhat surprising, the contribution of the larger offsets did not make any noticeable difference to the final migrated stack. However, larger differences were observed in the velocity analysis resolution, as described in the next section.

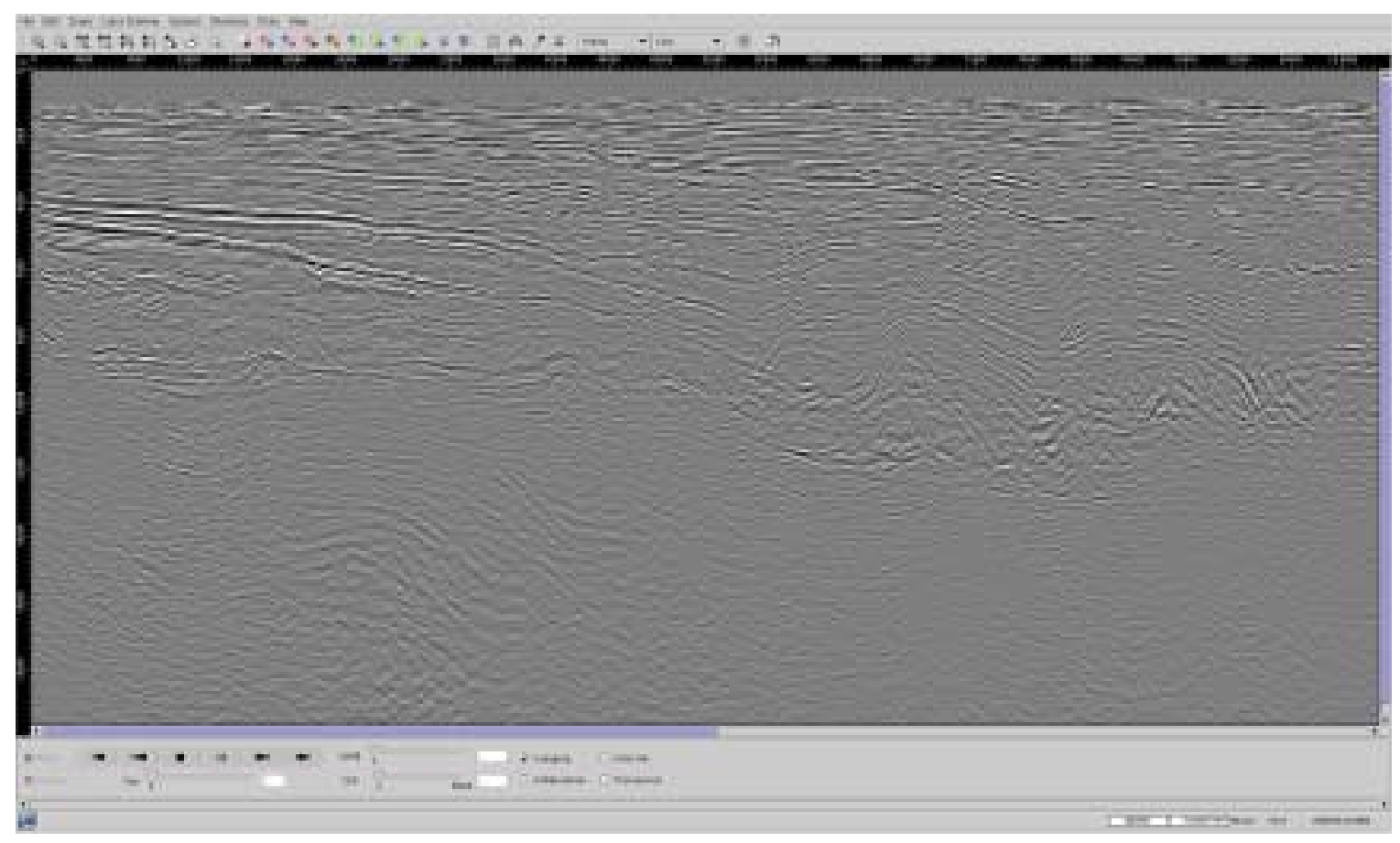

Figure 6.1: Kirchhoff migration with 40 degrees migration aperture. Data courtesy of PGS. 


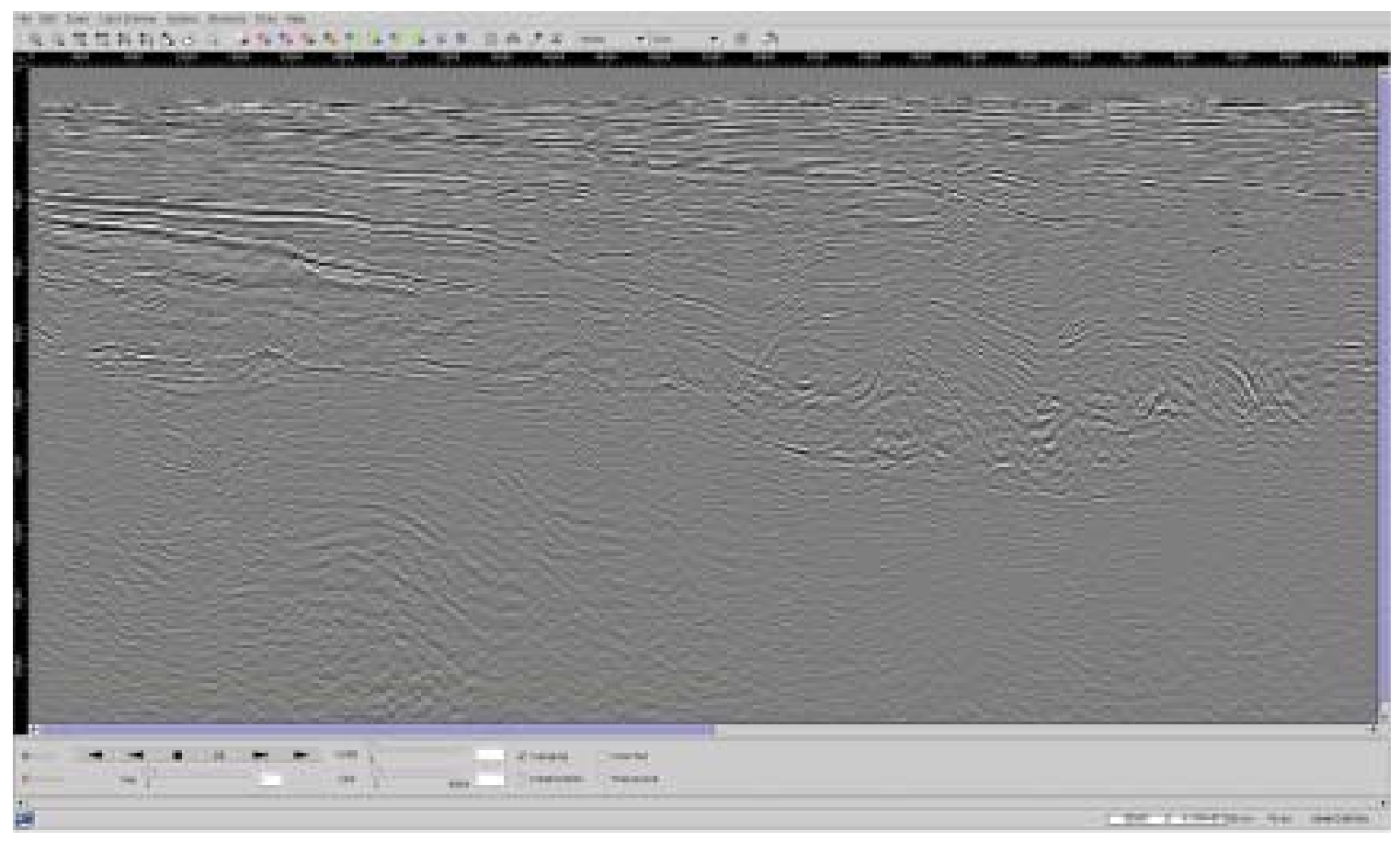

Figure 6.2: Kirchhoff migration with 80 degrees migration aperture. Data courtesy of PGS.

\section{Task 7: Investigate the effect of large offsets on velocity discrimination on ultra-deep data.}

To investigate the effect of large offsets on ultra-deep data, two additional datasets were created using the original input data by restricting the offset to 4000 meters and 6000 meters. These two datasets were migrated using the same parameters and velocity model used to migrate the full offset original input data. Figures 7.1 and 7.3 show the differences of the angle gathers using 4000, 6000 and 10,000 meters offsets, while Figure 7.2 and 7.4 show the corresponding residual semblance gathers. The larger offsets exhibit more continuity for some key reflectors, but at the same time there are unexplained new events coming from larger offsets that do not fit the typical kinematics. The energy from these new events improve the semblance analysis in the deeper areas enabling a more precise definition of the velocity model which in turn enables a better focusing of the events in these deeper areas. However, the improvements seem to be secondary and these benefits should be weighted against the increased costs of large offset acquisition. The more probable benefits would come from the additional amplitude vs. offset or amplitude vs. angle information, but this analysis is beyond the scope of this project. 

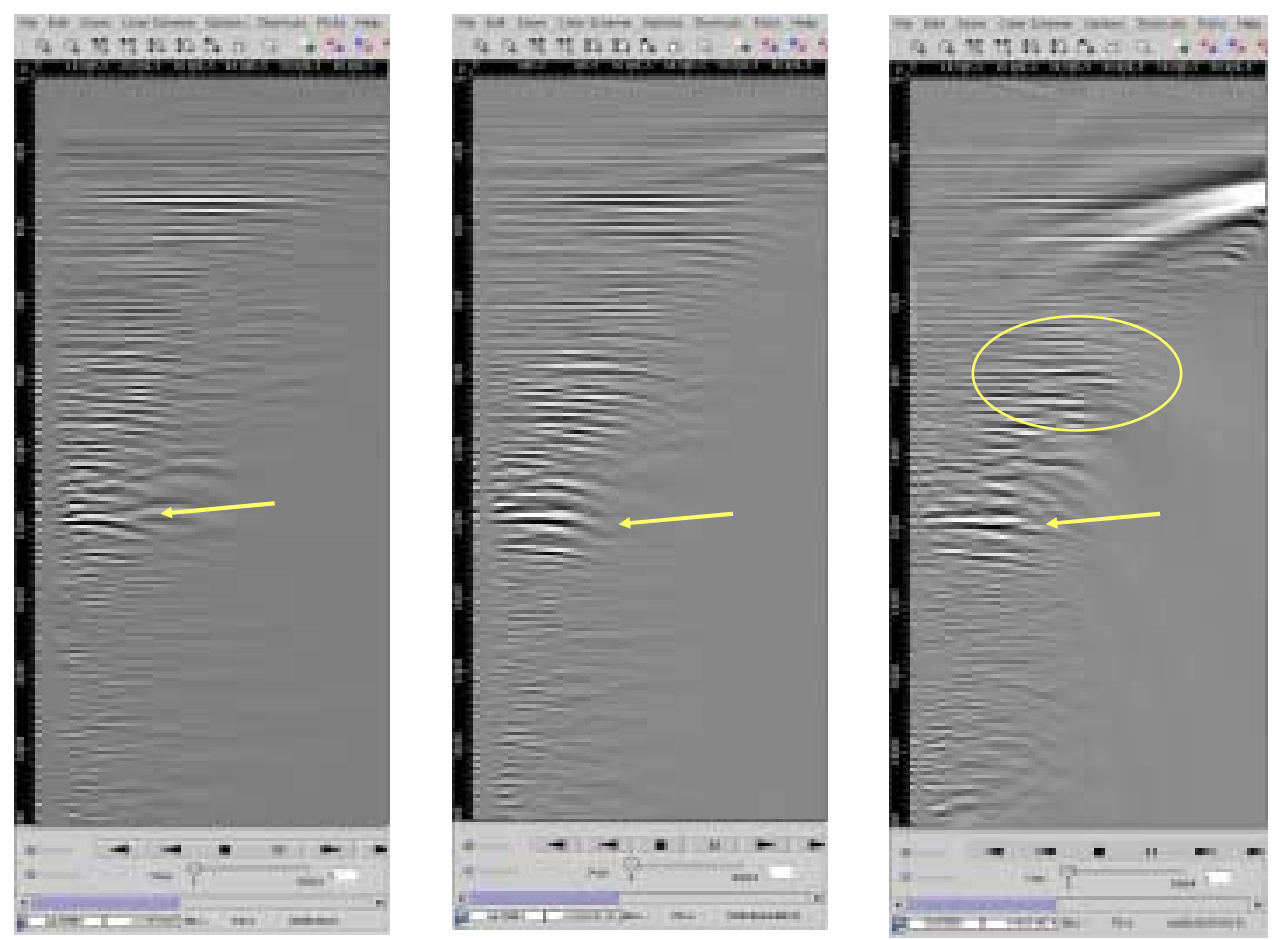

Figure 7.1: Angle gathers using 4000 meters offset, 6000 meters offset and 10,000 meters offset.
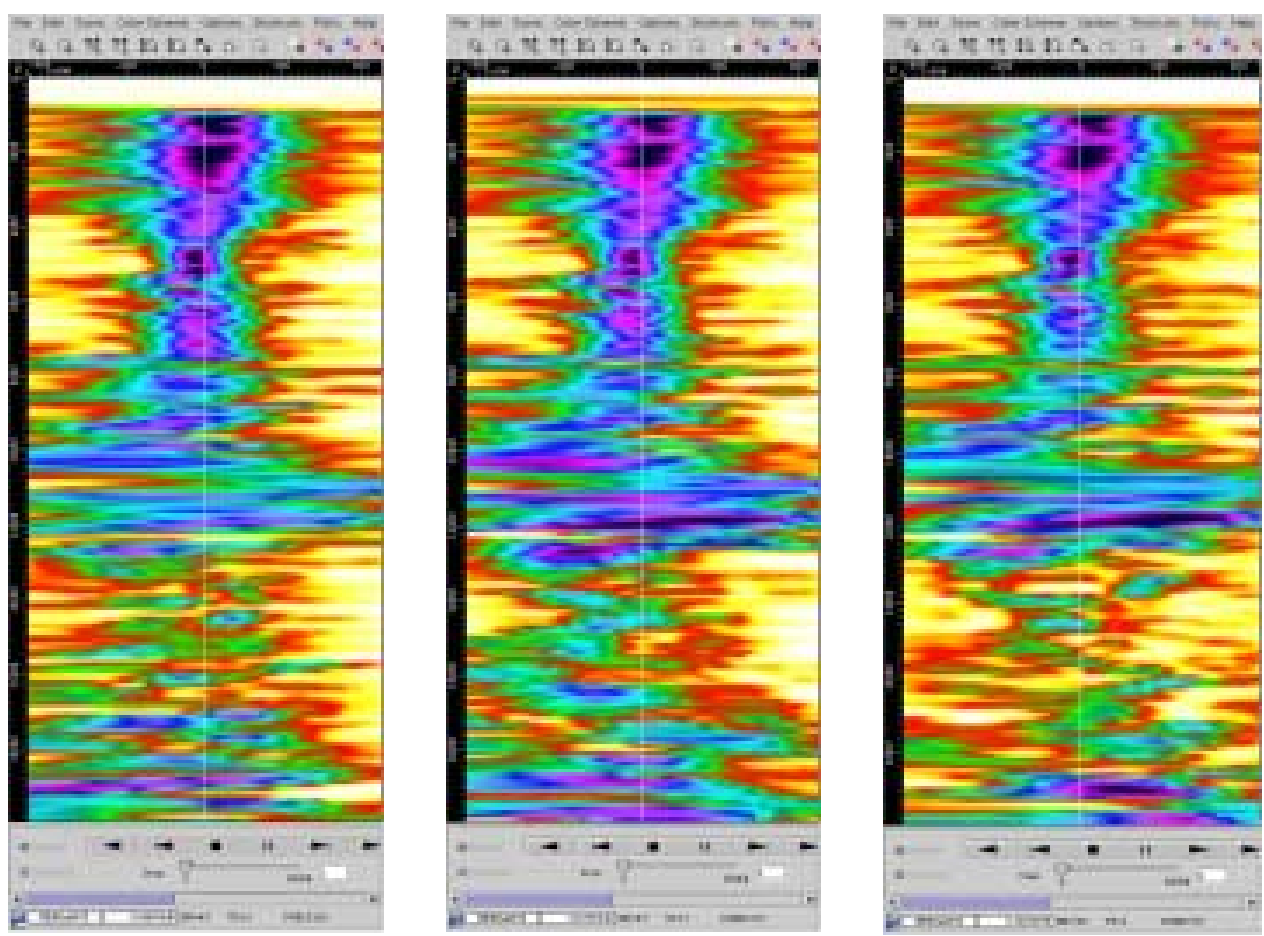

Figure 7.2: Residual semblance panels for the angle gathers using 4000 meters offset, 6000 meters offset and 10,000 meters offset. 

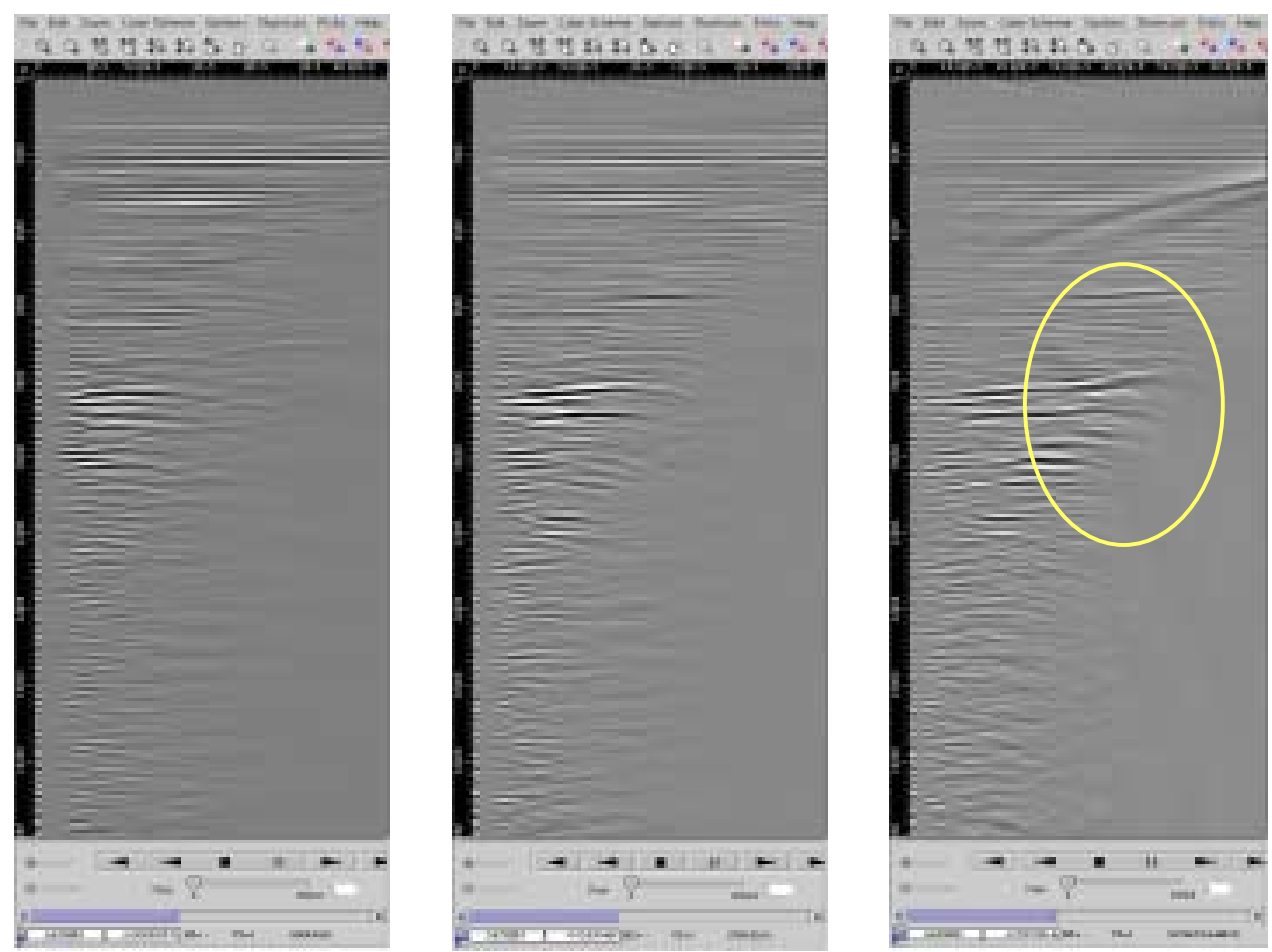

Figure 7.3: Angle gathers using 4000 meters offset, 6000 meters offset and 10,000 meters offset.
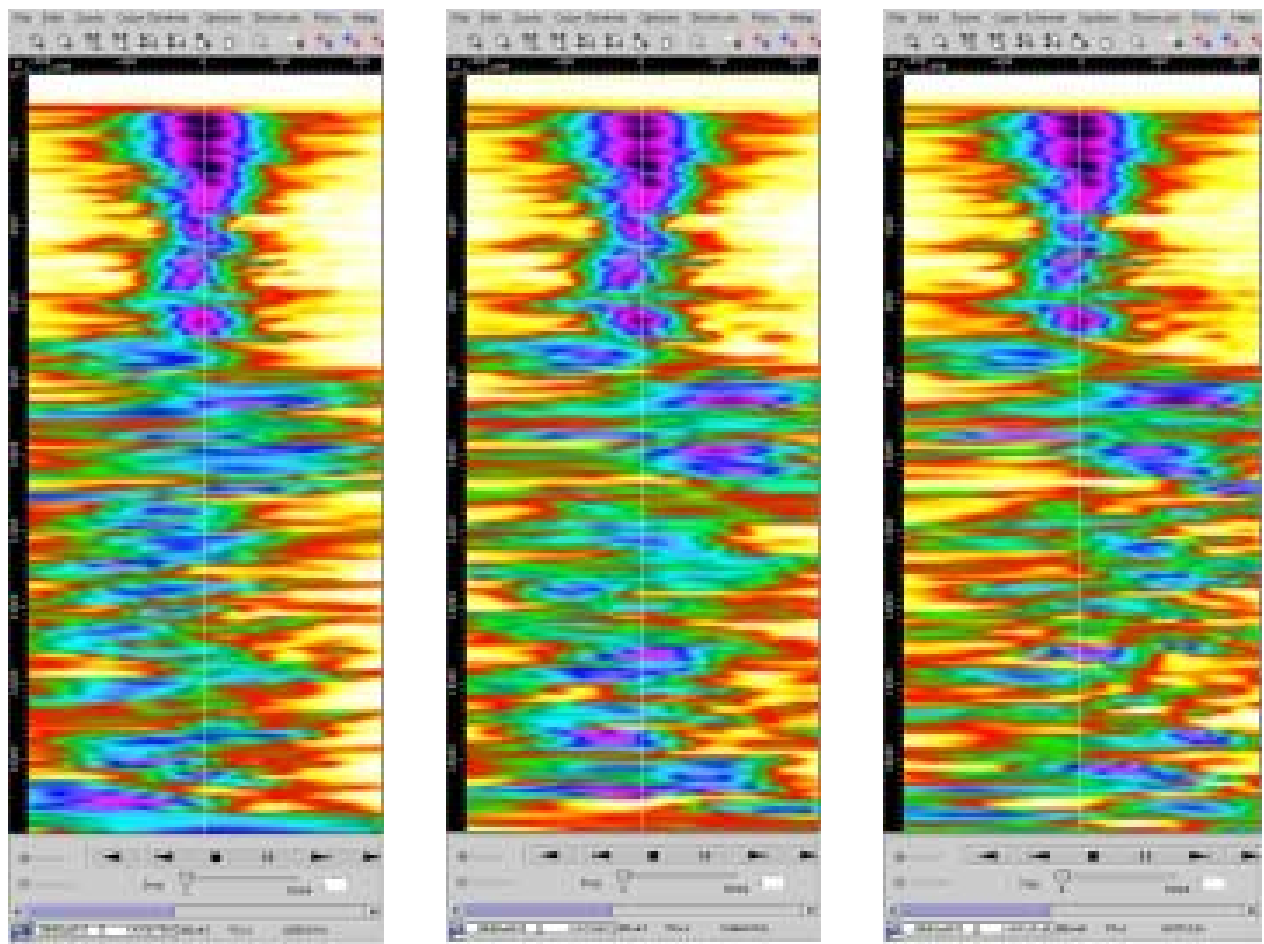

Figure 7.4: Residual semblance panels for the angle gathers using 4000 meters offset, 6000 meters offset and 10,000 meters offset. 


\section{CONCLUSIONS}

In this DoE NETL project we developed and tested a new technology designed to enhance seismic resolution and imaging of ultra-deep complex geologic structures by using (1) waveequation depth migration and (2) wave-equation velocity model building technology for deeper data penetration and recovery, steeper dip and ultra-deep structure imaging, and accurate velocity estimation for imaging and pore pressure prediction, and (3) accurate illumination and amplitude processing for extending the AVO prediction window. We addressed the theory of the handling true amplitudes in the downward continuation and imaging algorithm and the preservation of the amplitude with offset or amplitude with angle information required for AVO studies, the effect of several imaging conditions on amplitudes, non-elastic attenuation and approaches for recovering the amplitude and frequency, and the effect of aperture and illumination on imaging steep dips and on discriminating the velocities in the ultra-deep structures. The results on real data show that ultra-deep wave-equation imaging provides much greater resolution and accuracy than what can be accomplished with standard imaging technology. The advanced imaging methodology may improve the success rate and cost effectiveness for new deep-field discoveries, and also has applications in increasing recovery efficiency for the development of existing fields.

\section{REFERENCES}

Biondi, B., and Symes, W. W., 2004, Angle-domain common-image gathers for migration velocity analysis by wavefield-continuation imaging: Geophysics, 69, 1283-1298.

Blias, E., S. Gritsenko, and V. Chernjak, 1984, Traveltime derivatives in the layered medium: Soviet Geology and Geophysics, 75-81.

Claerbout, J. F., 1985, Imaging the Earth's Interior: Blackwell Scientific Publications.

Claerbout, J. F., 1970, Coarse grid calculations of waves in inhomogeneous media with application to delineation of complicated seismic structure: Geophysics, 35, 407-418.

Claerbout, J. F., 1993, Huygens waveforms and half-order derivatives, in SEP 77, 403-408. Stanford Exploration Project.

Docherty, P., 1991, A brief comparison of some Kirchhoff integral formulas for migration and inversion: Geophysics, 56, 1164-1169.

Fomel, S., 2000, Applications of plane-wave destructor filters: Stanford Exploration Project Report \# 105, 1-26.

Fomel, S., 2004, Theory of 3-D angle gathers in wave-equation imaging in 74th Ann. Internat. Mtg. Soc. of Expl. Geophys.

Goldin, S. V., 1987, Dynamic analysis of images in seismics: Soviet Geology and Geophysics, $28,84-93$.

Goldin, S. V., 1991, Decomposition of geometrical approximation of a reflected wave: Soviet Geology and Geophysics, 32, 110-119. 
Gritsenko, S. A., 1984, Time field derivatives: Soviet Geology and Geophysics, 25, 103-109.

Keho, T. H. and W. B. Beydoun, 1988, Paraxial ray Kirchhoff migration: Geophysics, 53, 15401546.

Paffenholz, J., McLain, B., Zaske, J., and Keliher, P., 2002, Subsalt multiple attenuation and imaging: Observations from the Sigsbee2B synthetic dataset in 72nd Ann. Internat. Mtg. Soc. of Expl. Geophys., 2122-2125.

Rickett, J. E., and Sava, P. C., 2002, Offset and angle-domain common image-point gathers for shot-profile migration: Geophysics, 67, 883-889.

Sava, P. C., and Fomel, S., 2005, Coordinate-independent angle-gathers for wave equation migration in 75th Ann. Internat. Mtg. Soc. of Expl. Geophys.

Sava, P. C., and Fomel, S., 2003, Angle-domain common-image gathers by wavefield continuation methods: Geophysics, 68, 1065-1074.

Tygel, M., J. Schleicher, and P. Hubral, 1994, Kirchhoff-Helmholtz theory in modeling and migration: Journal of Seismic Exploration, 3, 203-214. 